\title{
Synthesis of Unsymmetrical Diarylfumaronitriles via Tandem Michael Addition and Oxidation under $\mathrm{K}_{3} \mathrm{Fe}(\mathrm{CN})_{6} / \mathrm{O}_{2}$ System
}

Chongjiu Lu, ${ }^{\text {a }}$ Min Ye, ${ }^{*, a, b}$ Lipeng Long, ${ }^{a}$ Yue Zheng, ${ }^{a}$ Jiameng Liu, ${ }^{a}$ Yue Zhang, ${ }^{\text {Z }}$ hengwang Chen*,a

${ }^{a}$ Key Laboratory of Organo-Pharmaceutical Chemistry of Jiangxi Province, Gannan Normal University, Ganzhou 341000, PR China.

${ }^{\mathrm{b}}$ Key Laboratory of Chemical Biology and Traditional Chinese Medicine Research, Hunan Normal University, Changsha, Hunan 410081, PR China.

E-mail: yemin811@gnnu.edu.cn; chenzwang@126.com

\section{Supporting Information \\ List of Contents}

Table of contents

Table of contents

Copies of ${ }^{1} \mathrm{H}$ and ${ }^{13} \mathrm{C}$ NMR spectra .S2

X-ray crystallographic data S43 


\section{Copies of ${ }^{1} \mathrm{H}$ and ${ }^{13} \mathrm{C}$ NMR spectra}

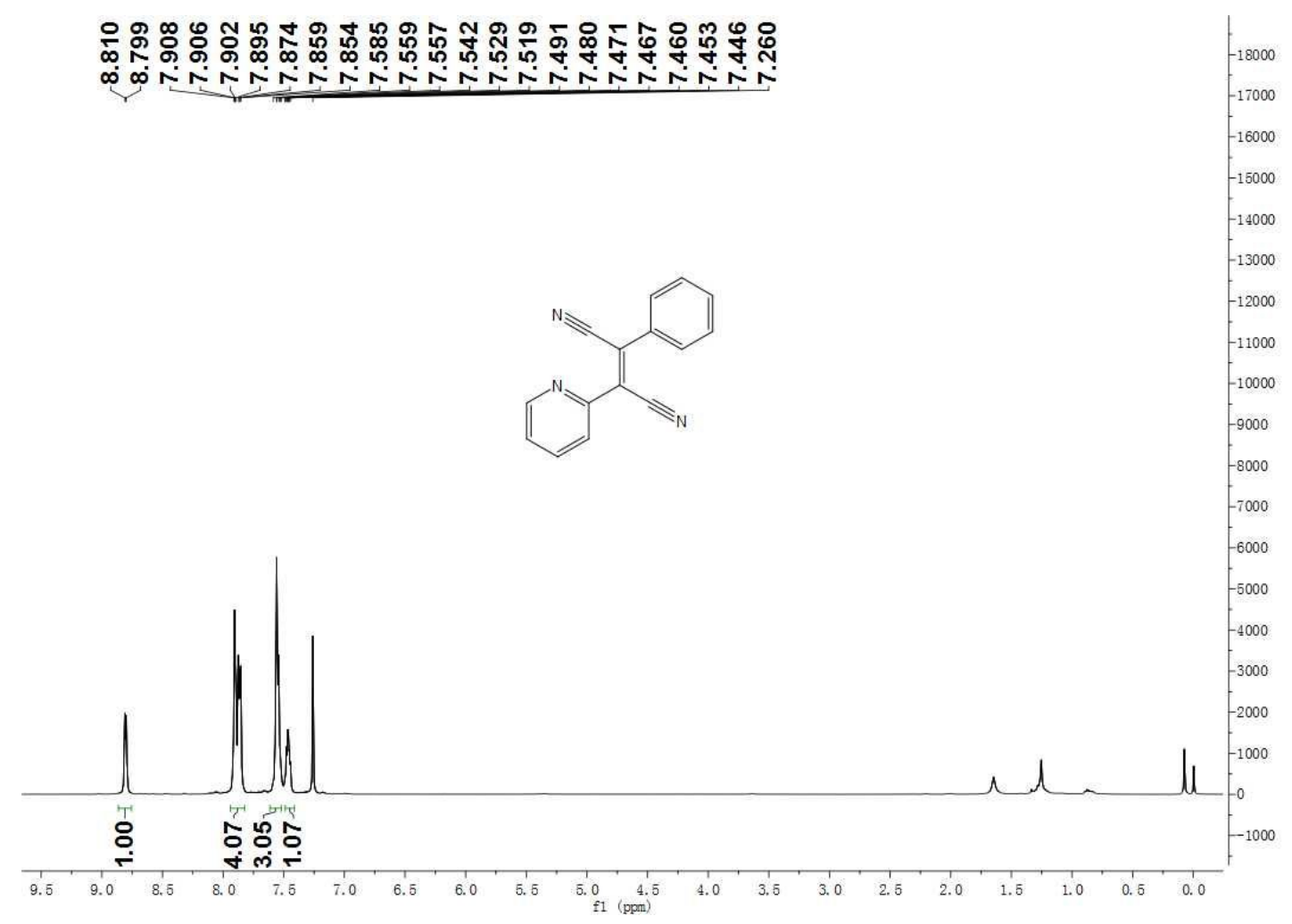

Figure S1. ${ }^{1} \mathrm{H}$ NMR spectrum of $\left.3 \mathrm{a}\left(400 \mathrm{MHz}^{\mathrm{CDCl}}\right)_{3}\right)$

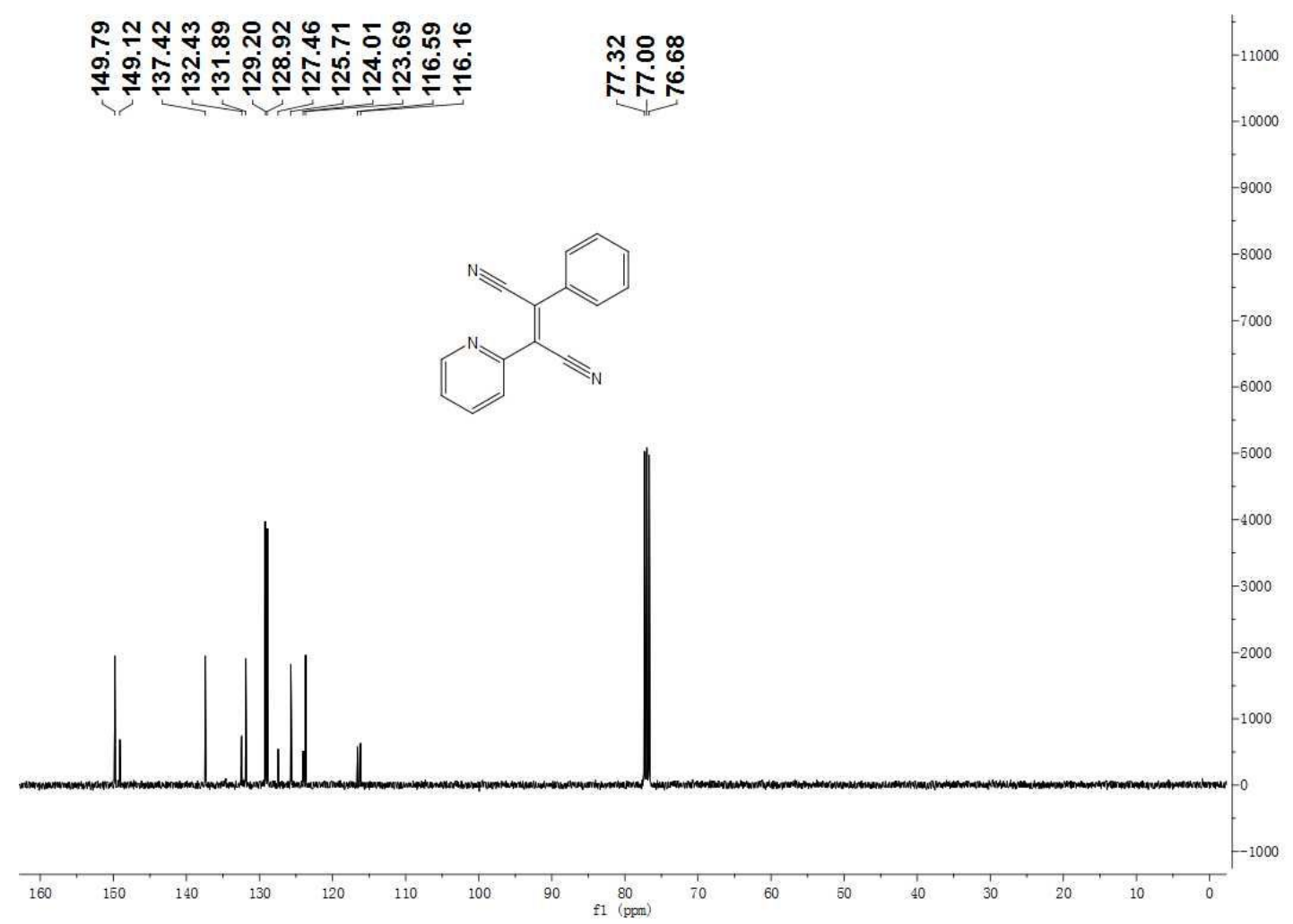

Figure S2. ${ }^{13} \mathrm{C}\left\{{ }^{1} \mathrm{H}\right\}$ NMR spectrum of $3 \mathrm{a}\left(100 \mathrm{MHz}, \mathrm{CDCl}_{3}\right)$ 


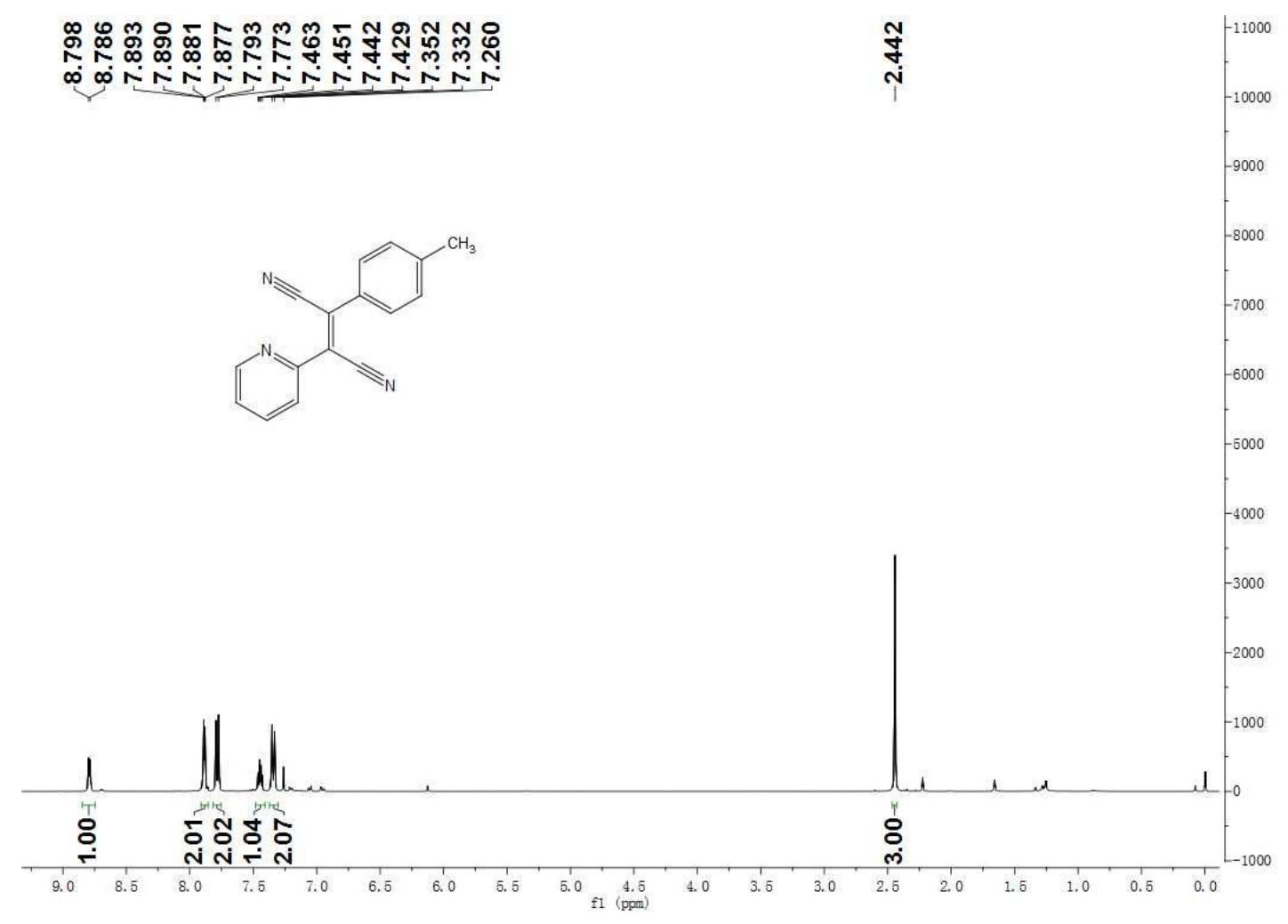

Figure S3. ${ }^{1} \mathrm{H}$ NMR spectrum of $3 \mathrm{~b}\left(400 \mathrm{MHz}, \mathrm{CDCl}_{3}\right)$

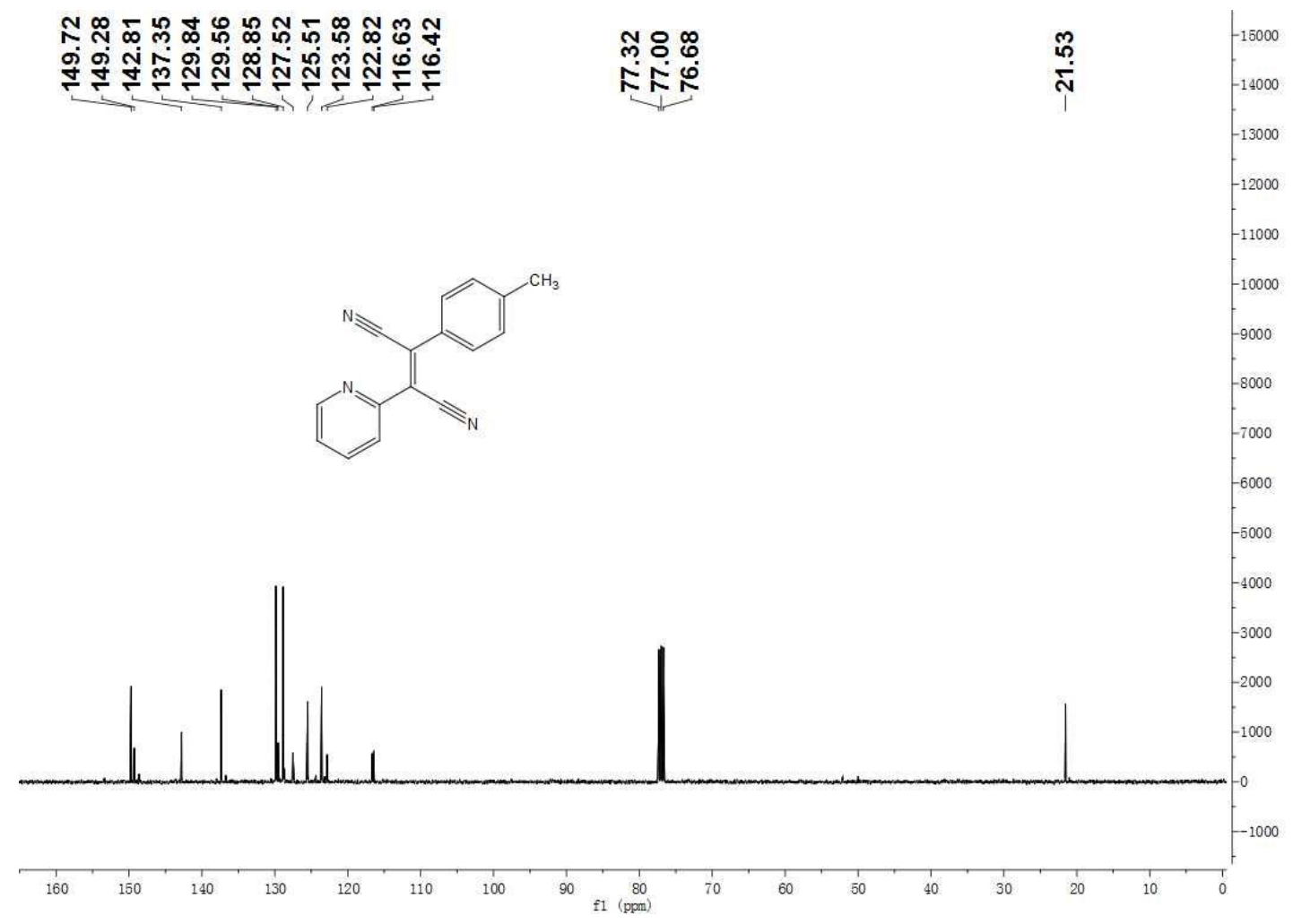

Figure S4. ${ }^{13} \mathrm{C}\left\{{ }^{1} \mathrm{H}\right\}$ NMR spectrum of $3 \mathrm{~b}\left(100 \mathrm{MHz}, \mathrm{CDCl}_{3}\right)$ 


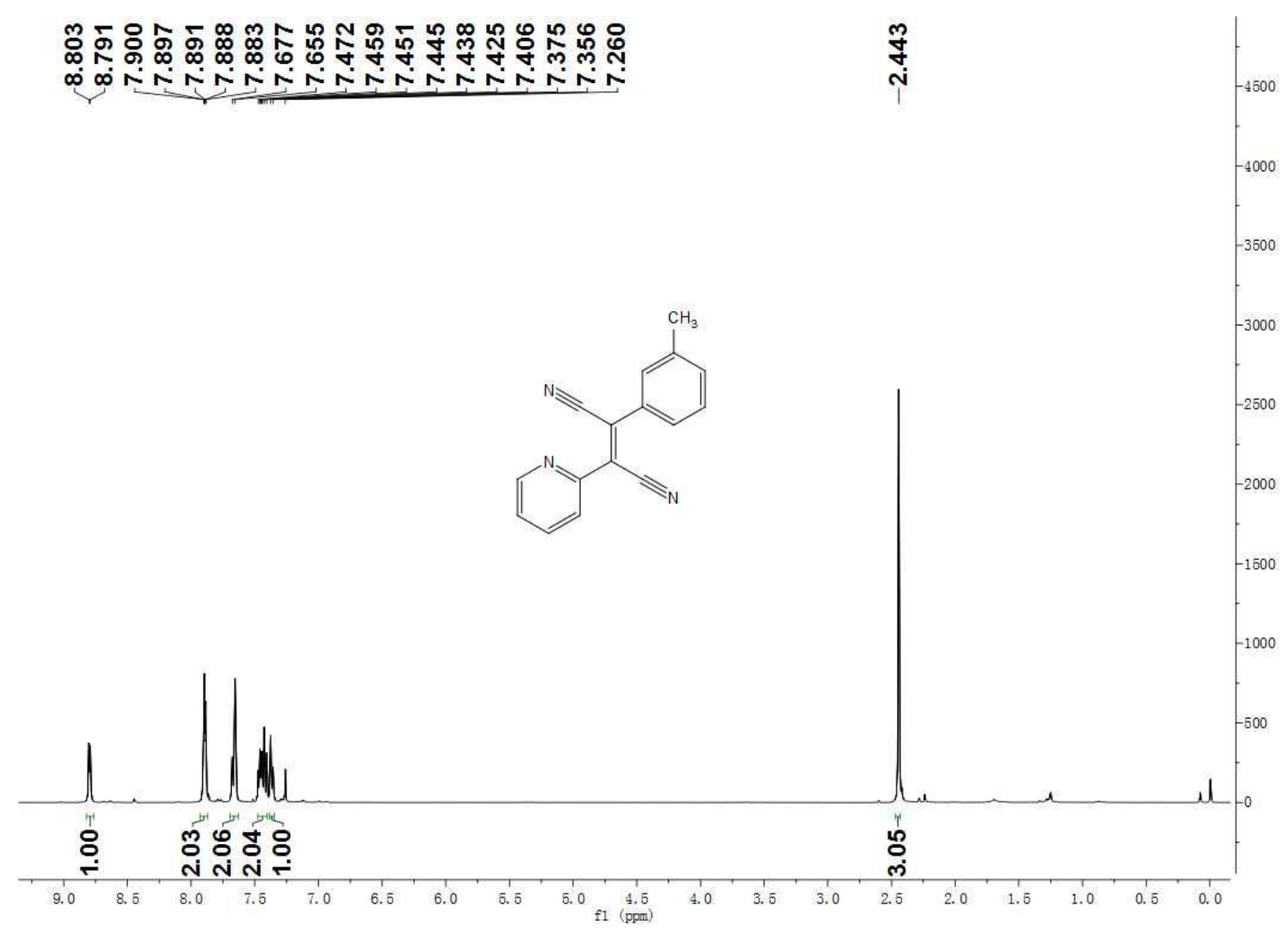

Figure S5. ${ }^{1} \mathrm{H}$ NMR spectrum of $3 \mathrm{c}\left(400 \mathrm{MHz}, \mathrm{CDCl}_{3}\right)$

$\kappa$

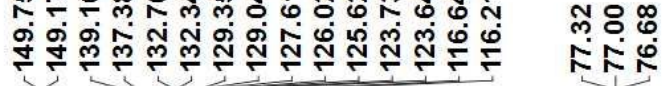

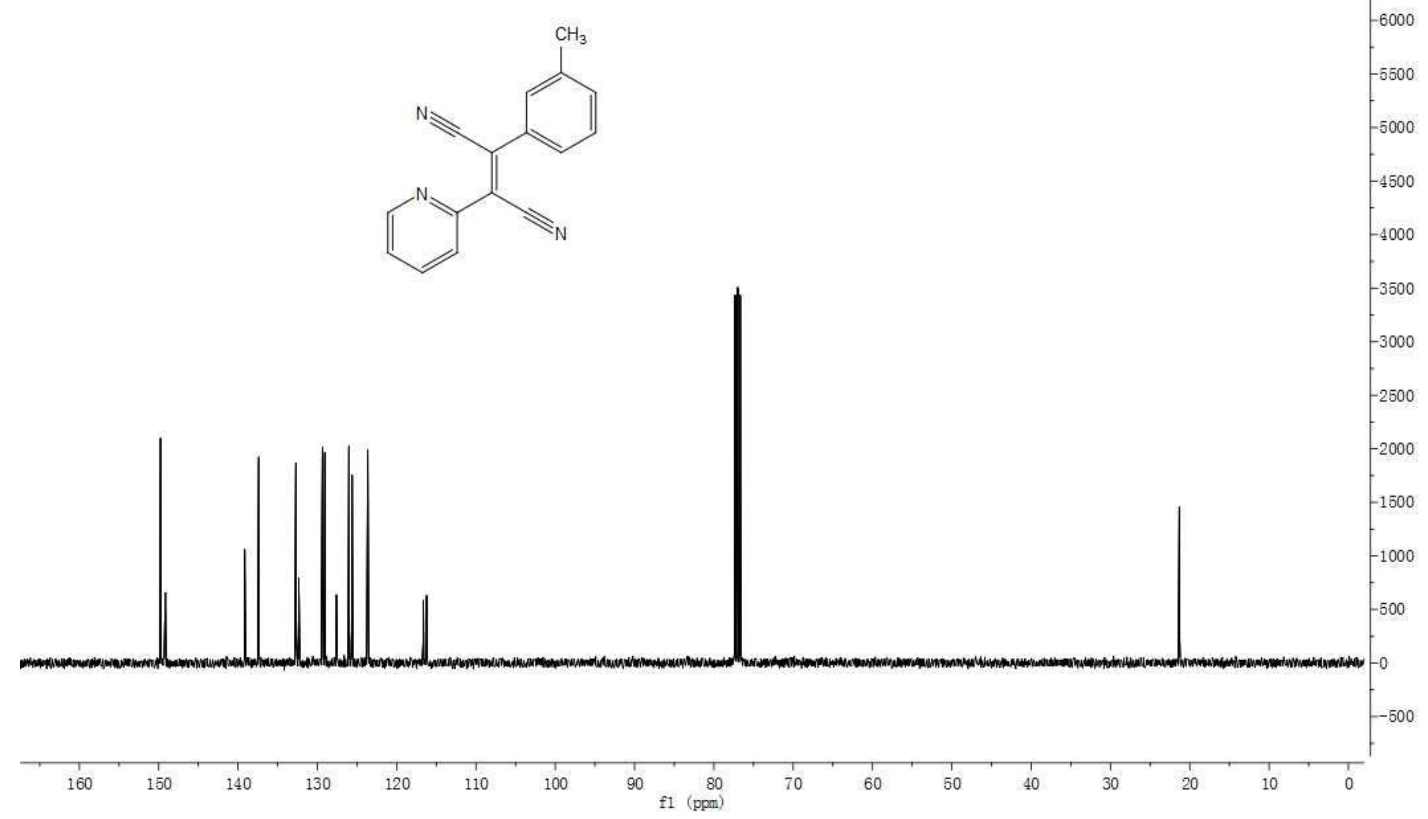

Figure S6. ${ }^{13} \mathrm{C}\left\{{ }^{1} \mathrm{H}\right\}$ NMR spectrum of $3 \mathrm{c}\left(100 \mathrm{MHz}, \mathrm{CDCl}_{3}\right)$ 


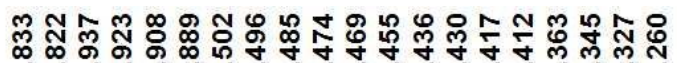

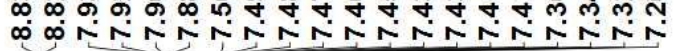

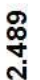<smiles>Cc1ccccc1C(C#N)=C(C#N)c1ccccn1</smiles>

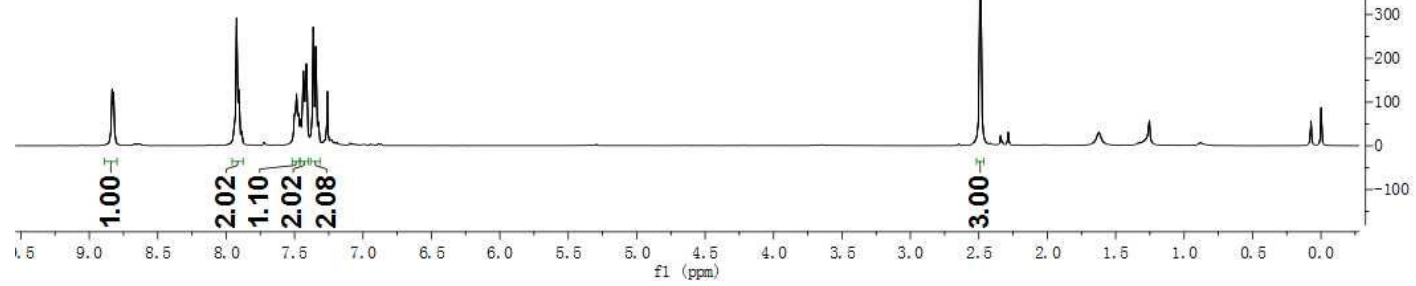

Figure S7. ${ }^{1} \mathrm{H}$ NMR spectrum of $3 \mathrm{~d}\left(400 \mathrm{MHz} \mathrm{CDCl}_{3}\right)$

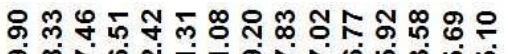

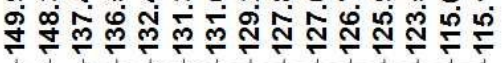

స్లి:

Ríg

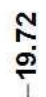

$-3800$

T-2 N

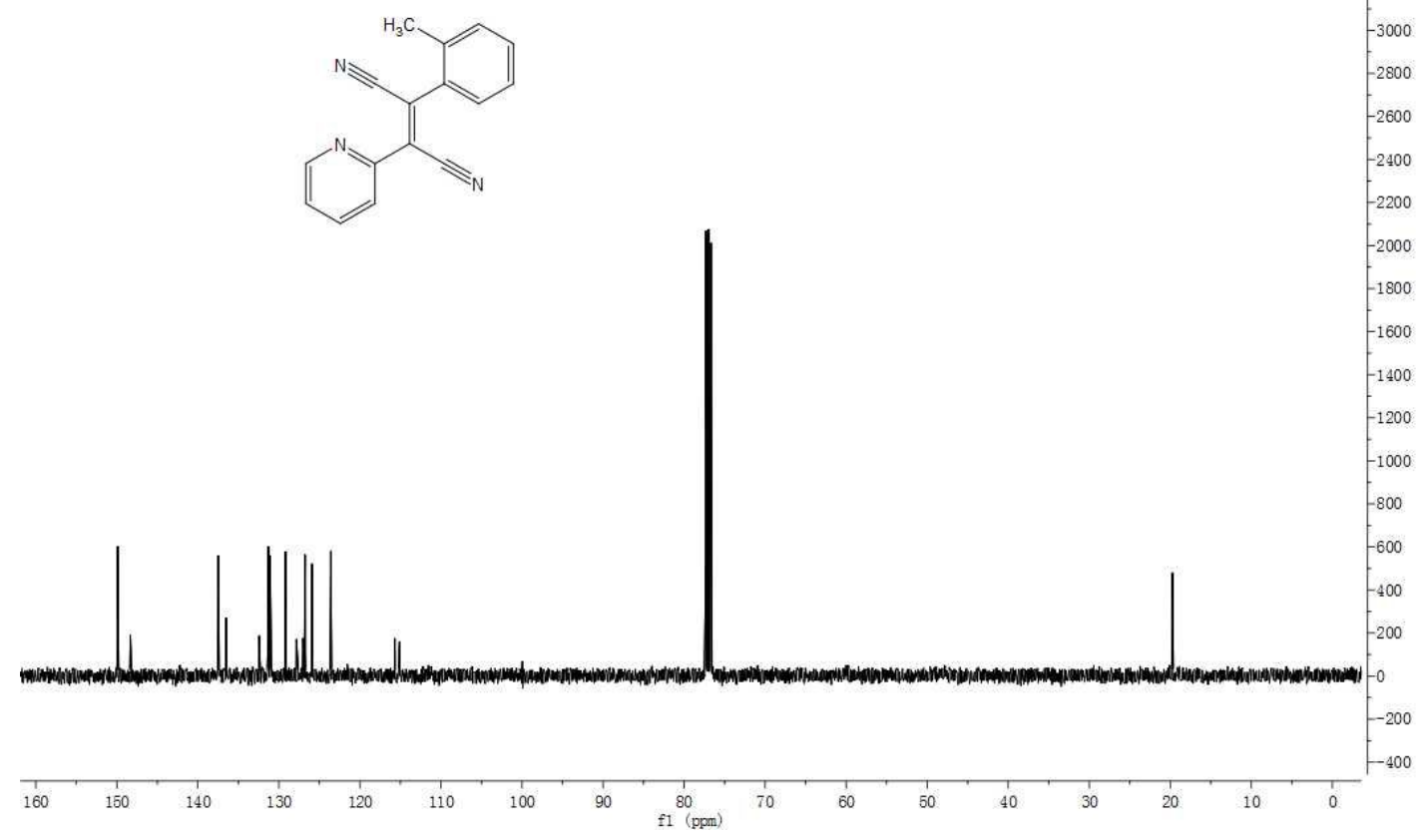

Figure S8. ${ }^{13} \mathrm{C}\left\{{ }^{1} \mathrm{H}\right\}$ NMR spectrum of $3 \mathrm{~d}\left(100 \mathrm{MHz}, \mathrm{CDCl}_{3}\right)$ 


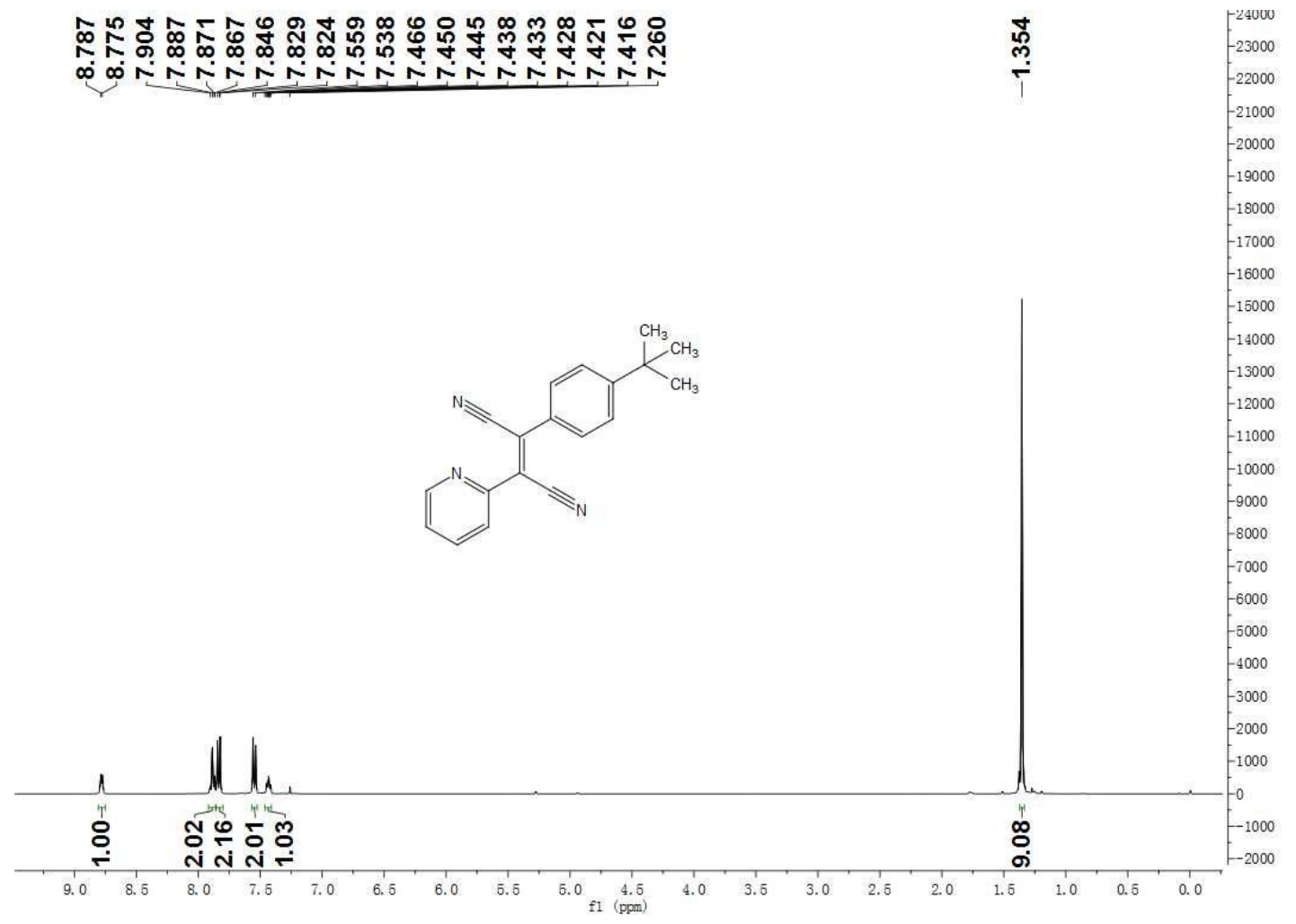

Figure S9. ${ }^{1} \mathrm{H}$ NMR spectrum of $3 \mathrm{e}\left(400 \mathrm{MHz}, \mathrm{CDCl}_{3}\right)$

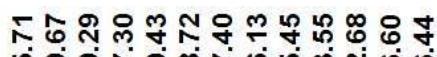

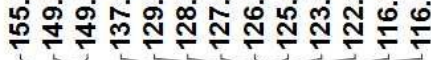
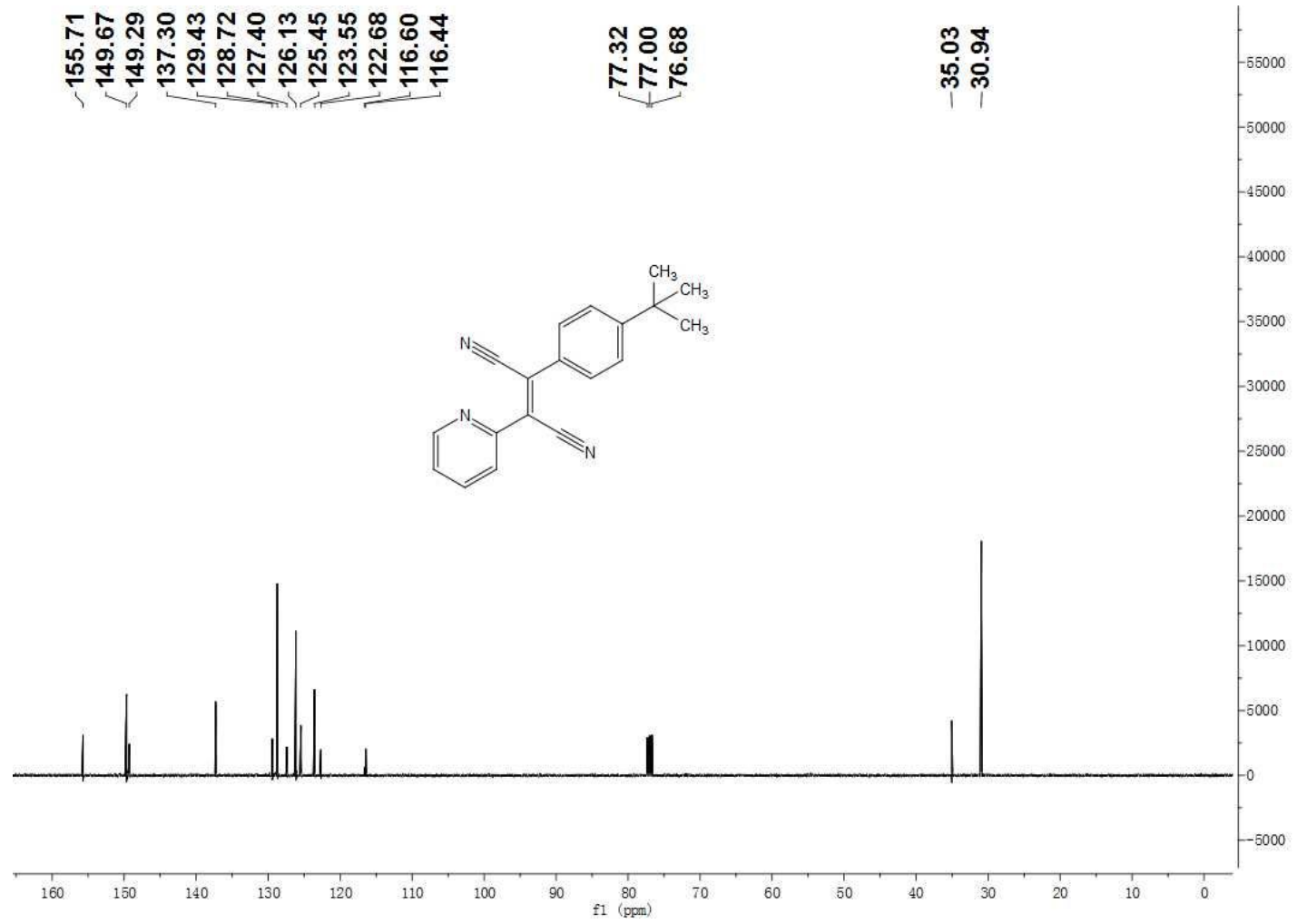

Figure S10. ${ }^{13} \mathrm{C}\left\{{ }^{1} \mathrm{H}\right\}$ NMR spectrum of $3 \mathrm{e}\left(100 \mathrm{MHz}, \mathrm{CDCl}_{3}\right)$ 


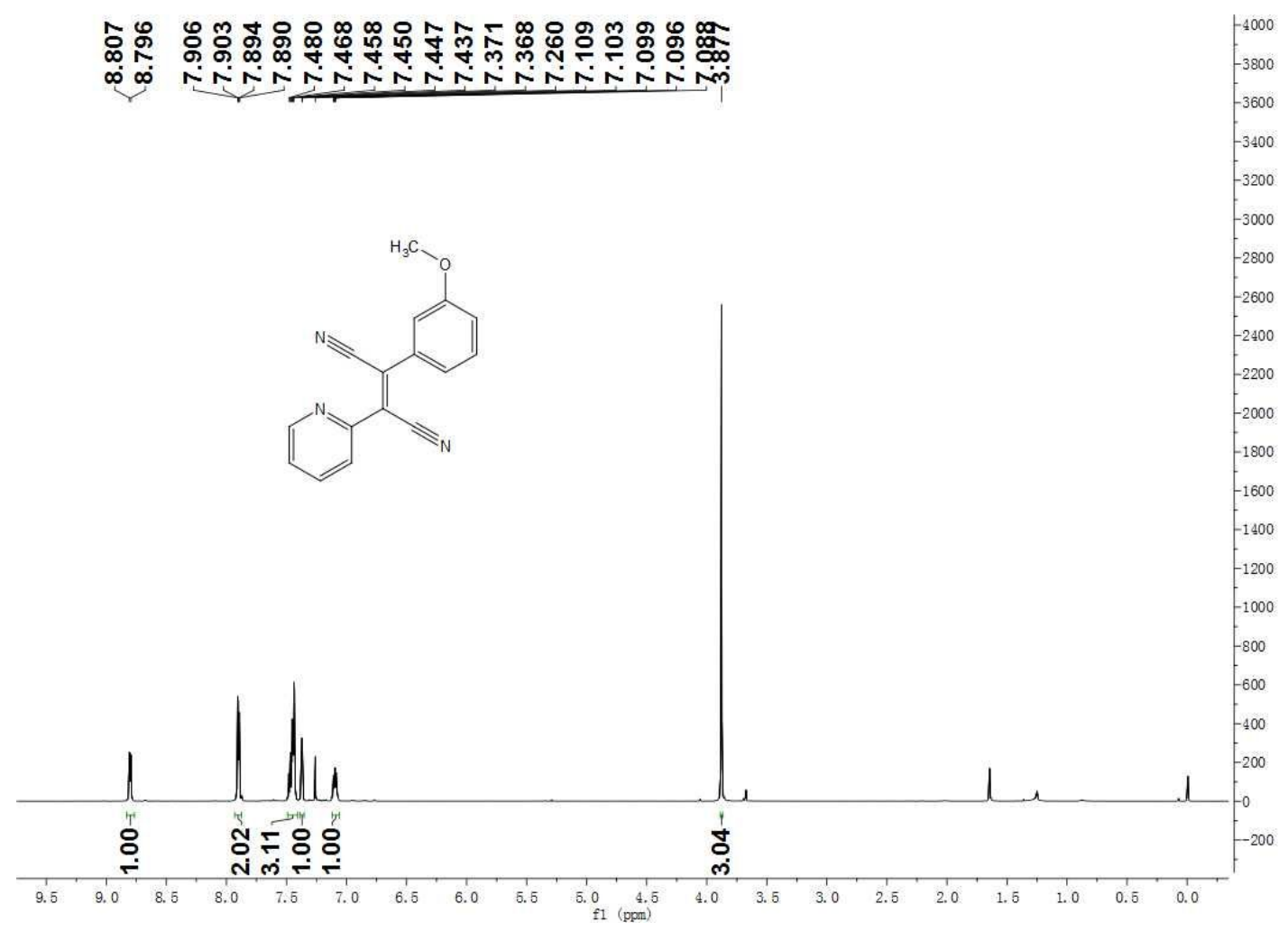

Figure S11. ${ }^{1} \mathrm{H}$ NMR spectrum of $3 f\left(400 \mathrm{MHz} \mathrm{CDCl}_{3}\right)$

๓

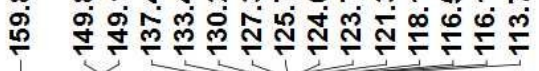

กำ:

사요

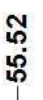

$-20000$

$-19000$

$-18000$

$-17000$

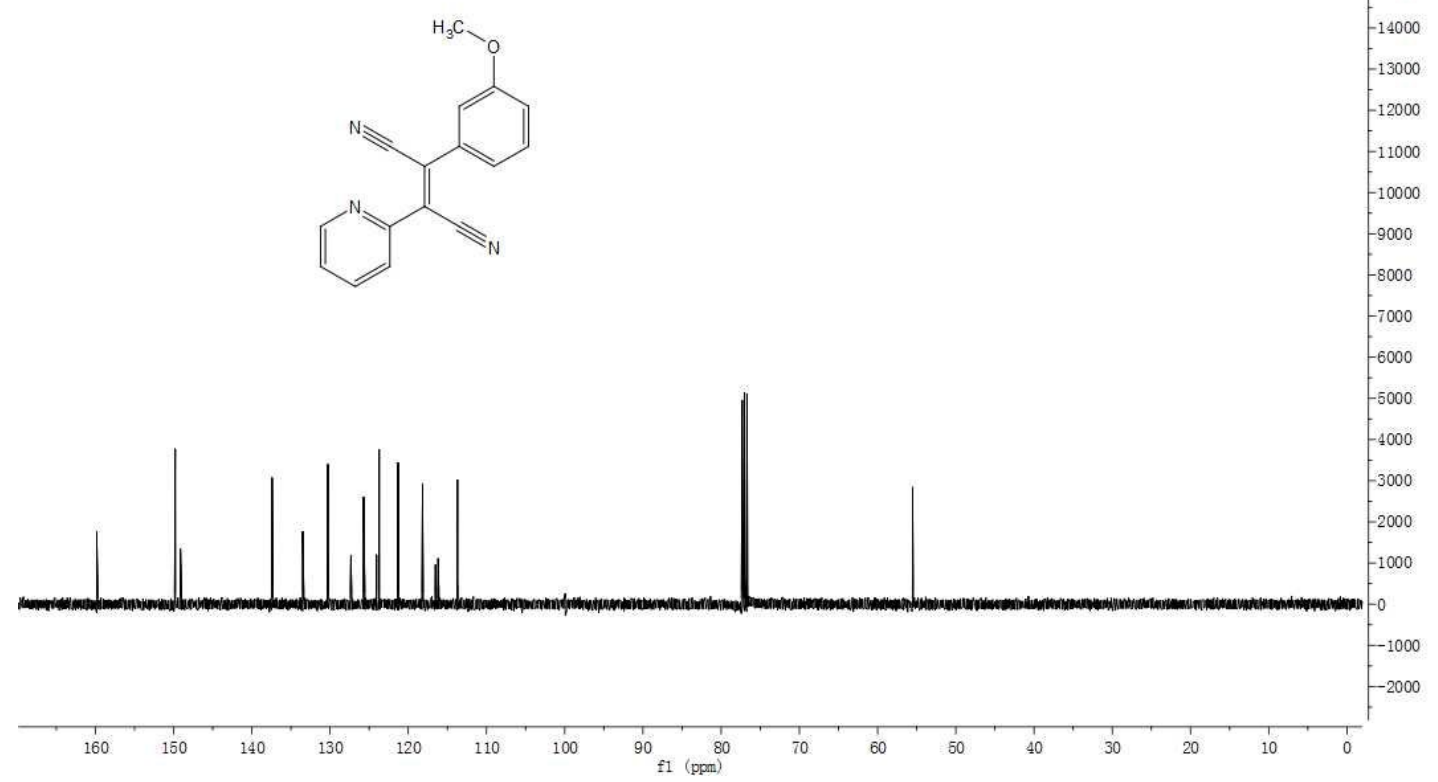

$-15000$

Figure S12. ${ }^{13} \mathrm{C}\left\{{ }^{1} \mathrm{H}\right\}$ NMR spectrum of $3 f\left(100 \mathrm{MHz}, \mathrm{CDCl}_{3}\right)$ 


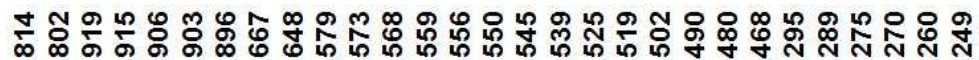

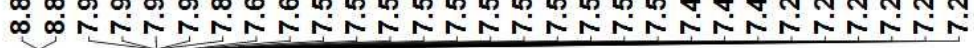
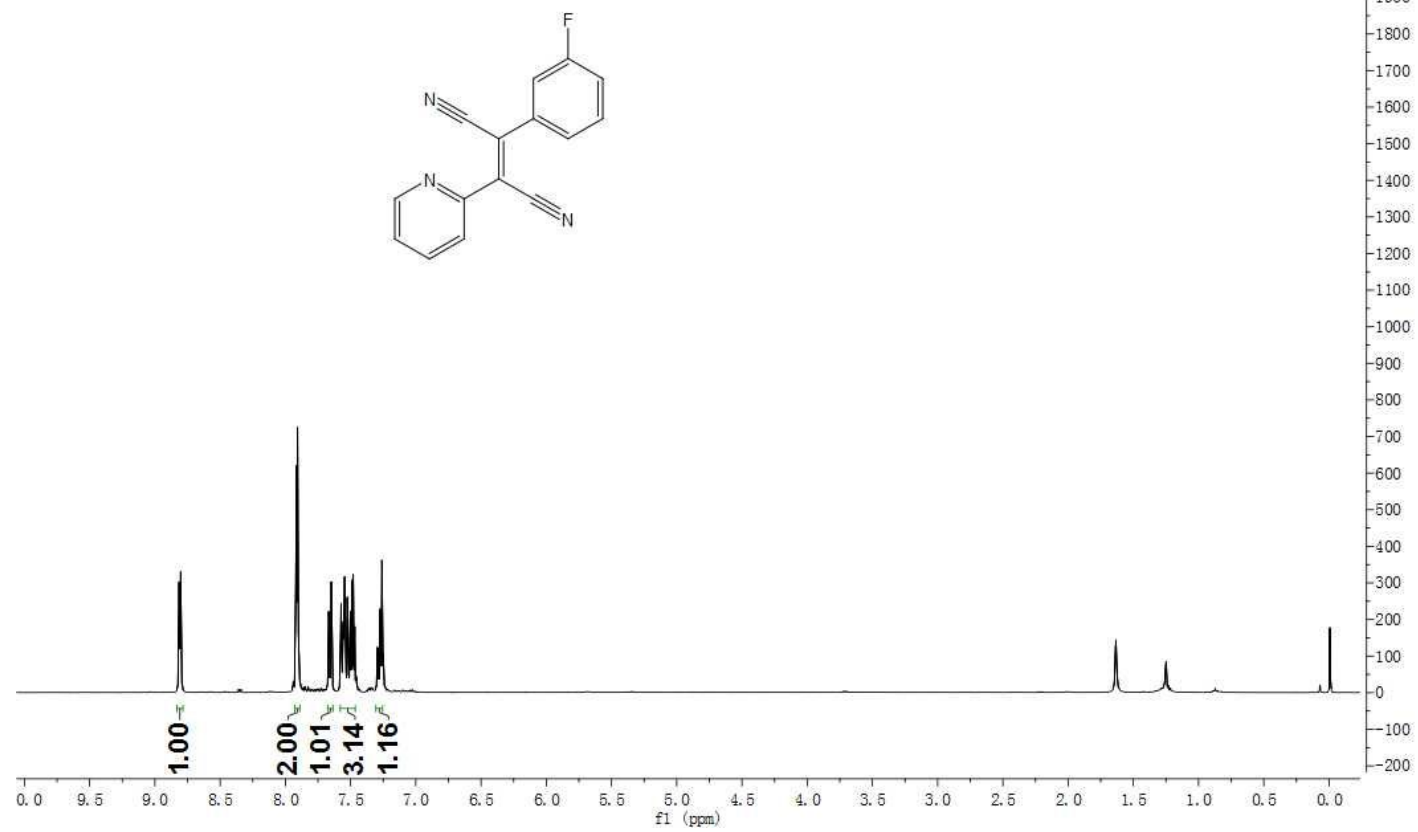

Figure S13. ${ }^{1} \mathrm{H}$ NMR spectrum of $3 \mathrm{~g}\left(400 \mathrm{MHz}, \mathrm{CDCl}_{3}\right)$

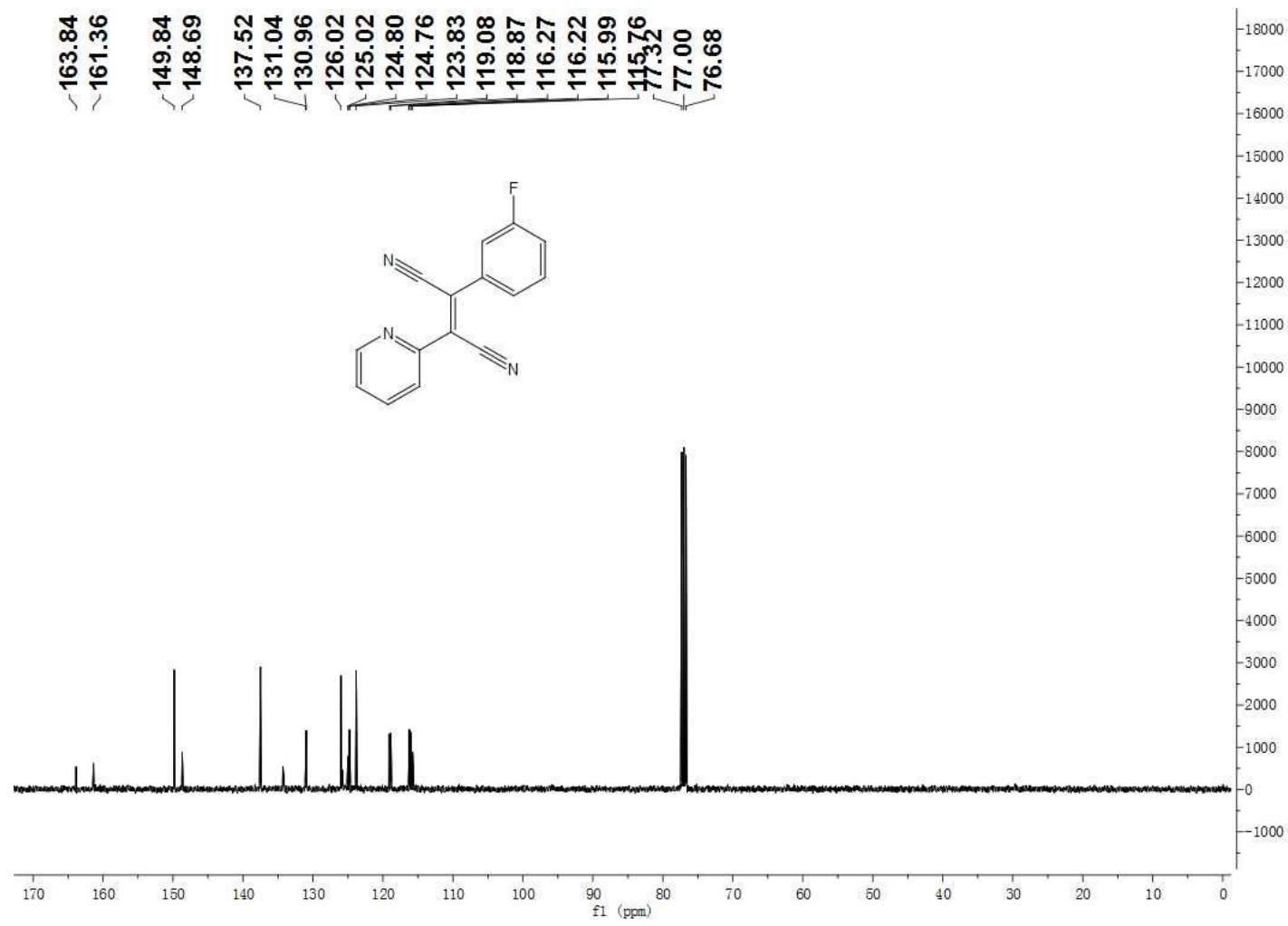

Figure S14. ${ }^{13} \mathrm{C}\left\{{ }^{1} \mathrm{H}\right\}$ NMR spectrum of $3 \mathrm{~g}\left(100 \mathrm{MHz}, \mathrm{CDCl}_{3}\right)$ 


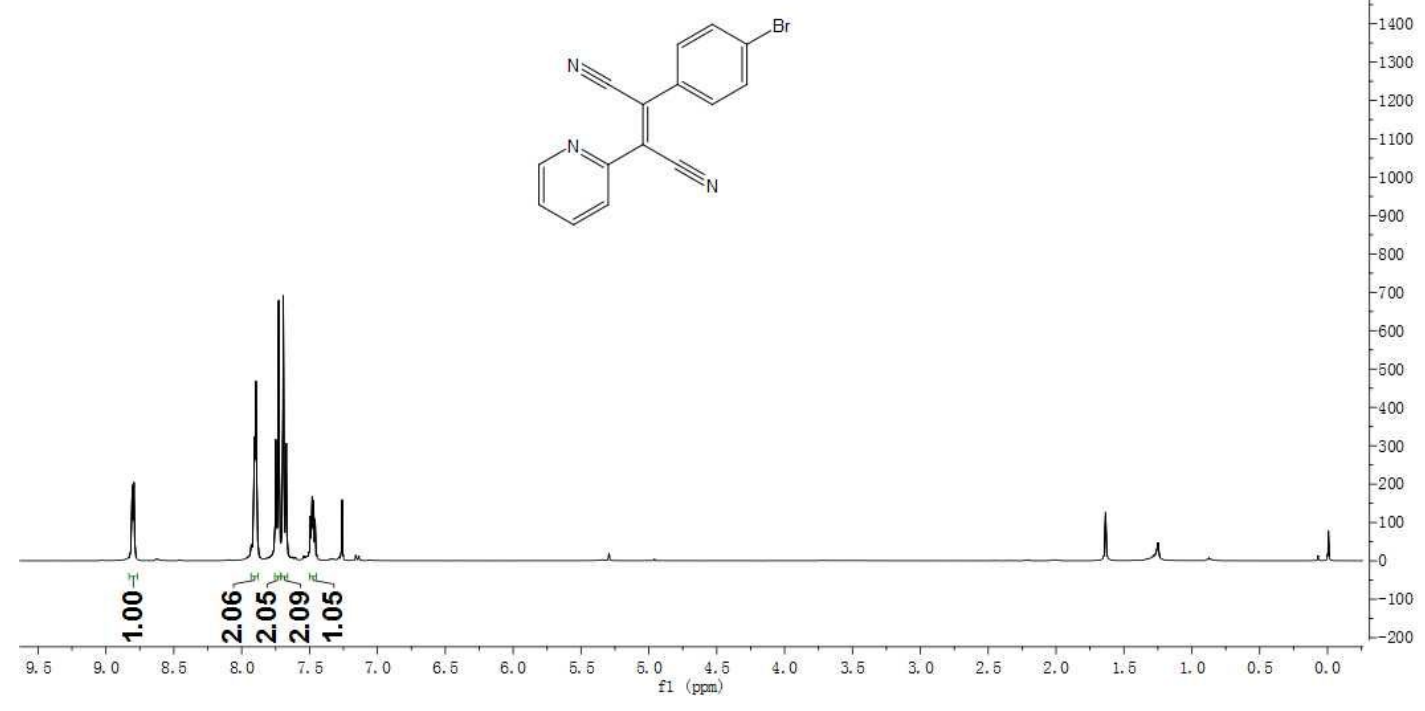

Figure S15. ${ }^{1} \mathrm{H}$ NMR spectrum of $3 \mathrm{~h}\left(400 \mathrm{MHz}, \mathrm{CDCl}_{3}\right)$

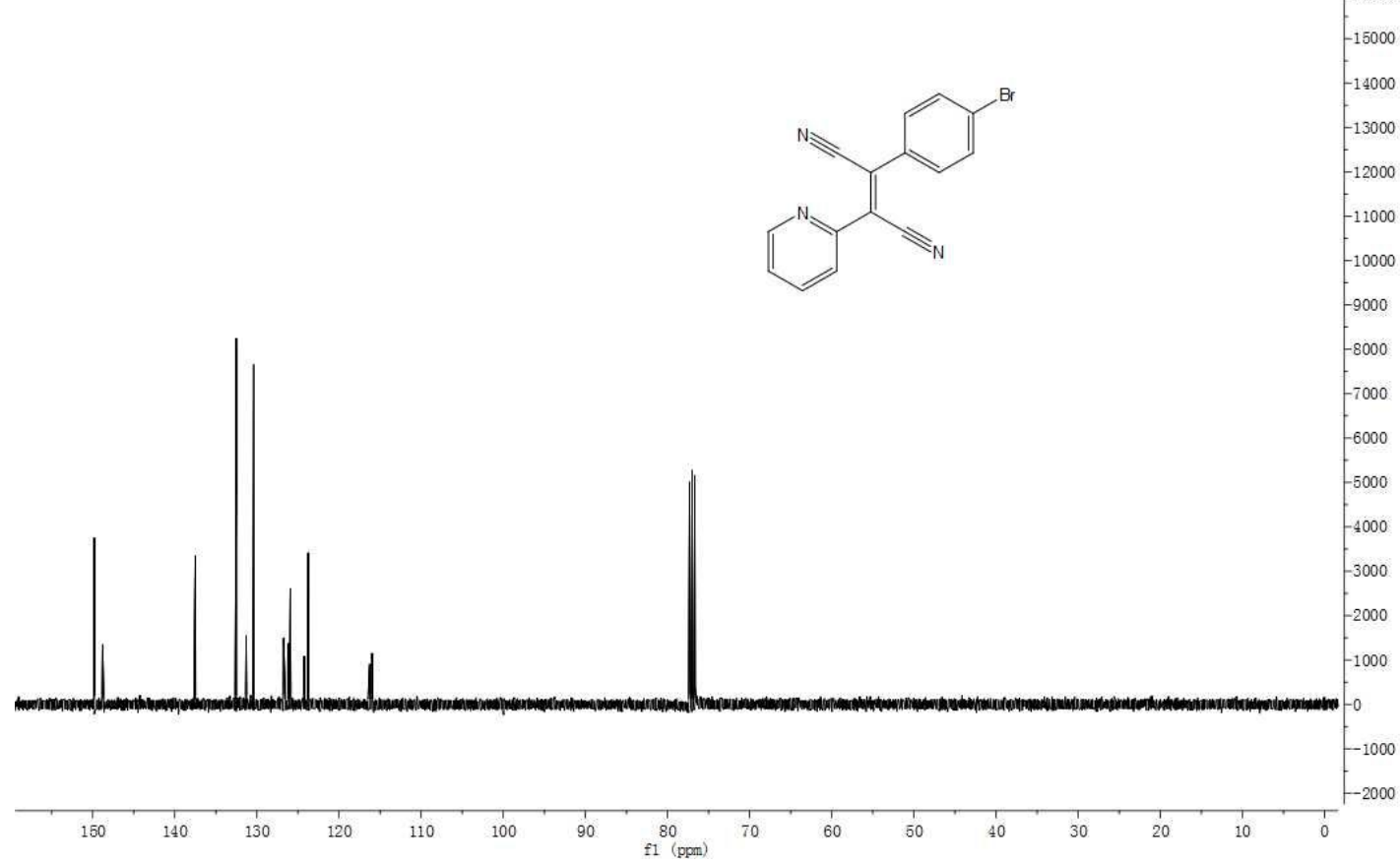

Figure S16. ${ }^{13} \mathrm{C}\left\{{ }^{1} \mathrm{H}\right\}$ NMR spectrum of $3 \mathrm{~h}\left(100 \mathrm{MHz}, \mathrm{CDCl}_{3}\right)$ 


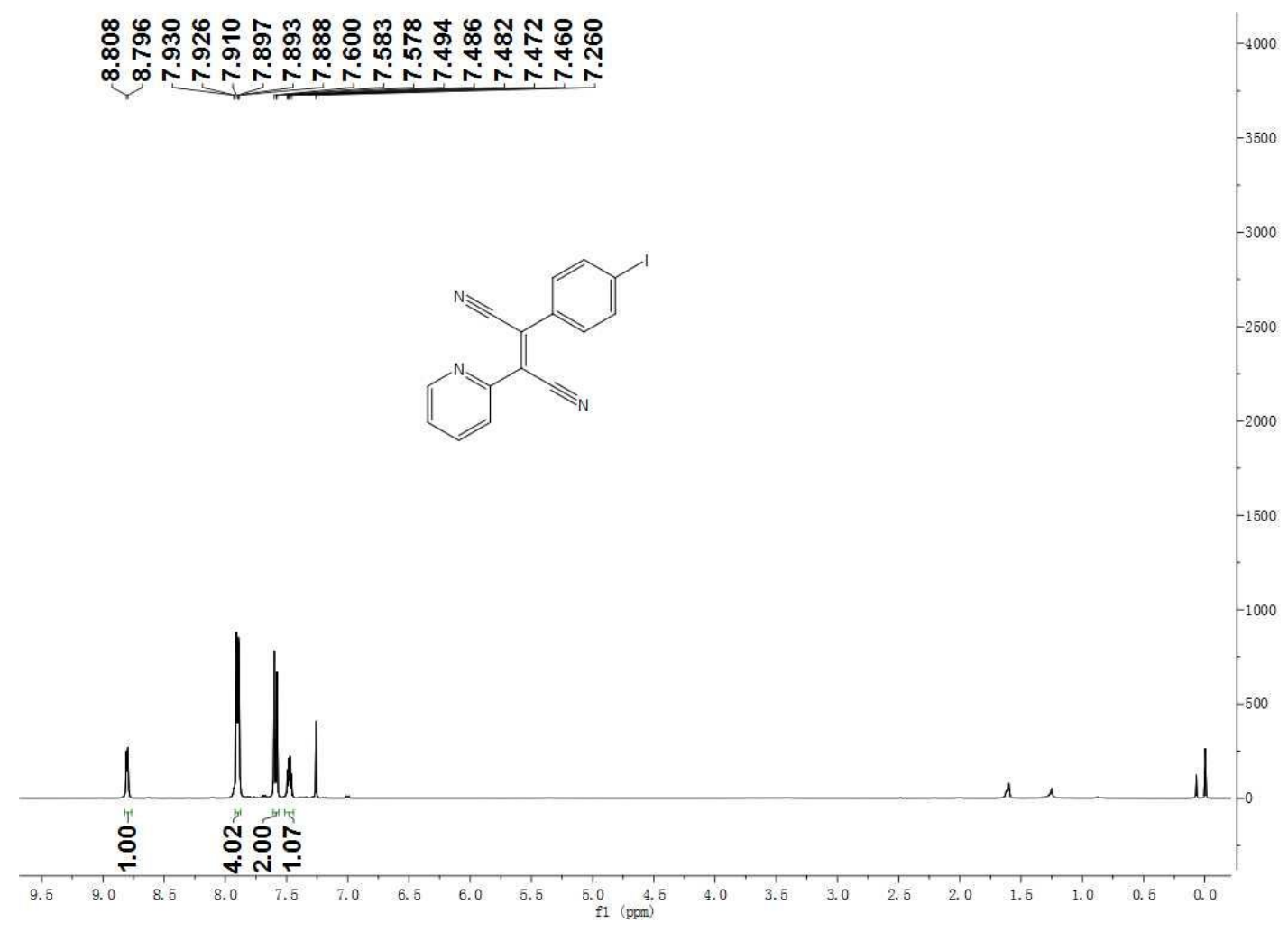

Figure S17. ${ }^{1} \mathrm{H}$ NMR spectrum of $3 \mathrm{i}\left(400 \mathrm{MHz}, \mathrm{CDCl}_{3}\right)$

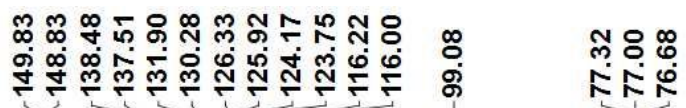

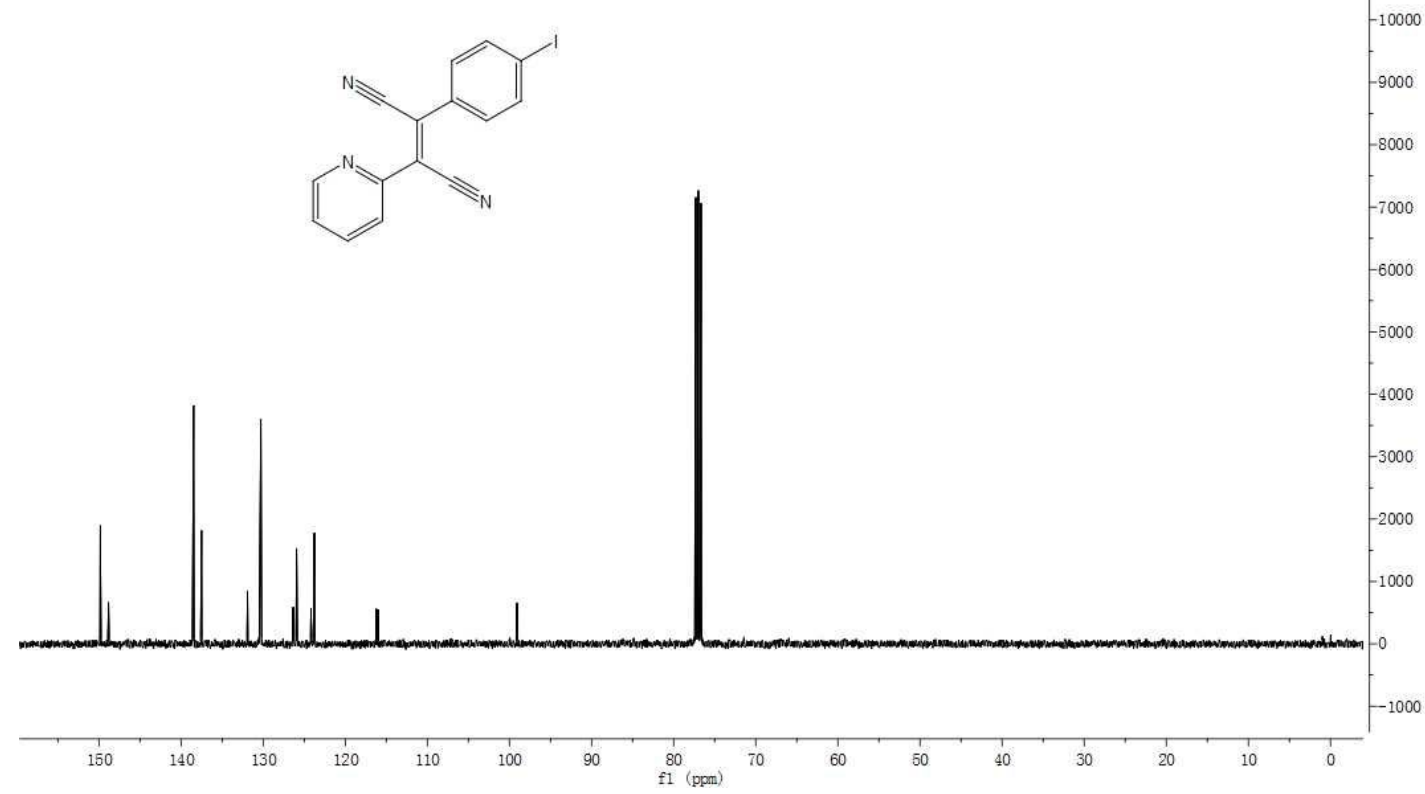

Figure S18. ${ }^{13} \mathrm{C}\left\{{ }^{1} \mathrm{H}\right\}$ NMR spectrum of $3 \mathrm{i}\left(100 \mathrm{MHz}, \mathrm{CDCl}_{3}\right)$ 


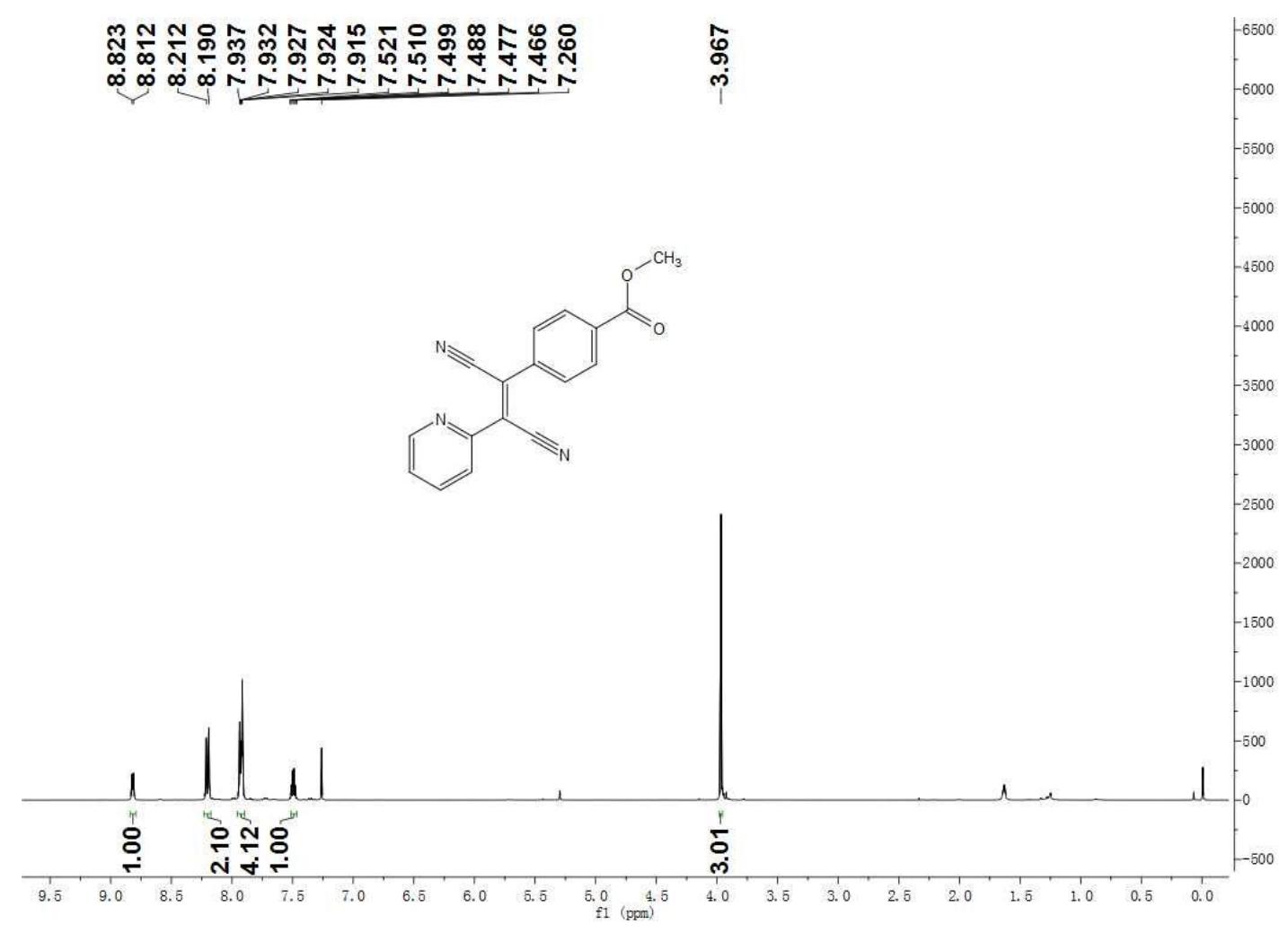

Figure S19. ${ }^{1} \mathrm{H}$ NMR spectrum of $3 \mathrm{j}\left(400 \mathrm{MHz}, \mathrm{CDCl}_{3}\right)$

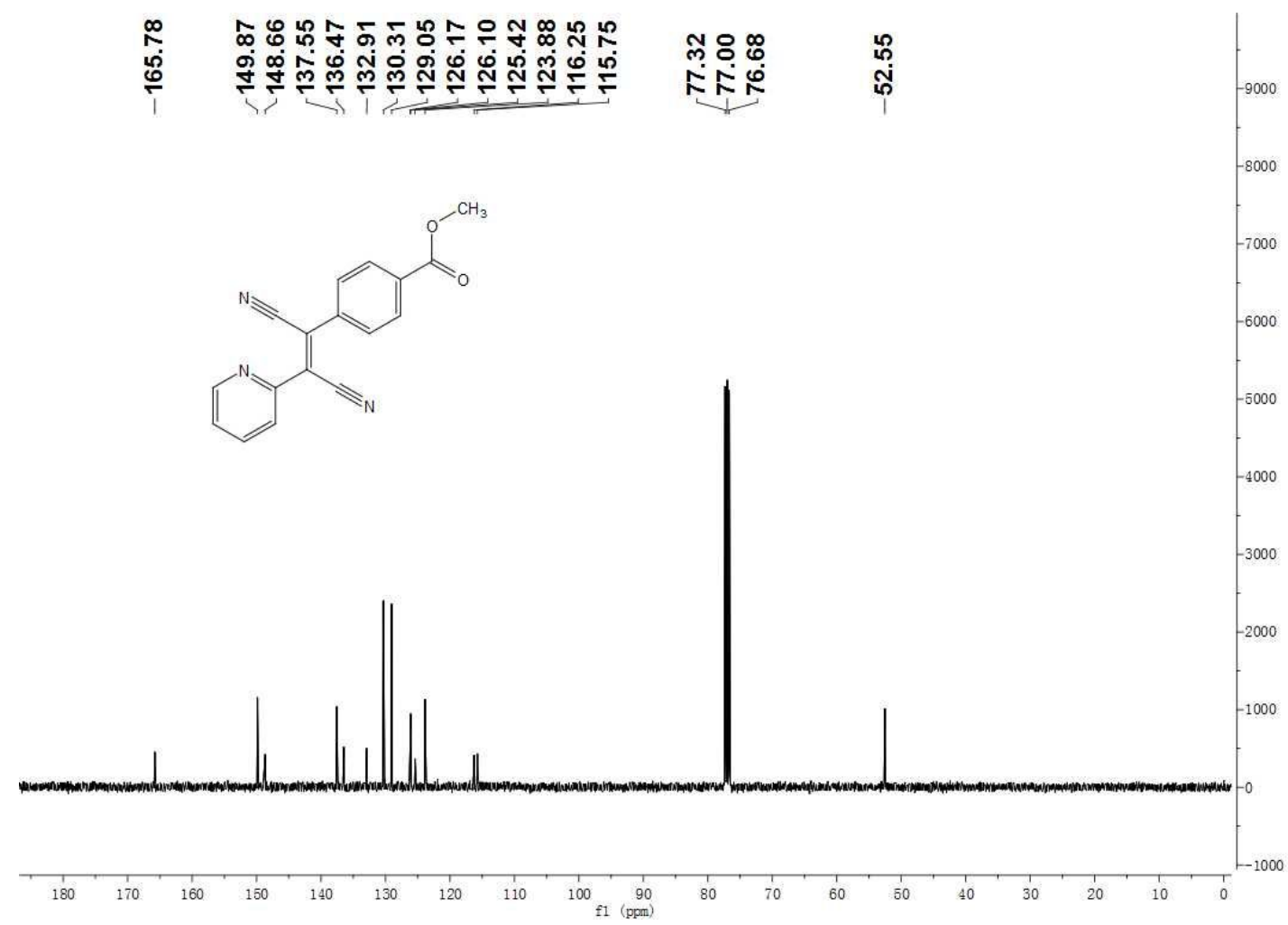

Figure S20. ${ }^{13} \mathrm{C}\left\{{ }^{1} \mathrm{H}\right\}$ NMR spectrum of $3 \mathrm{j}\left(100 \mathrm{MHz}, \mathrm{CDCl}_{3}\right)$ 


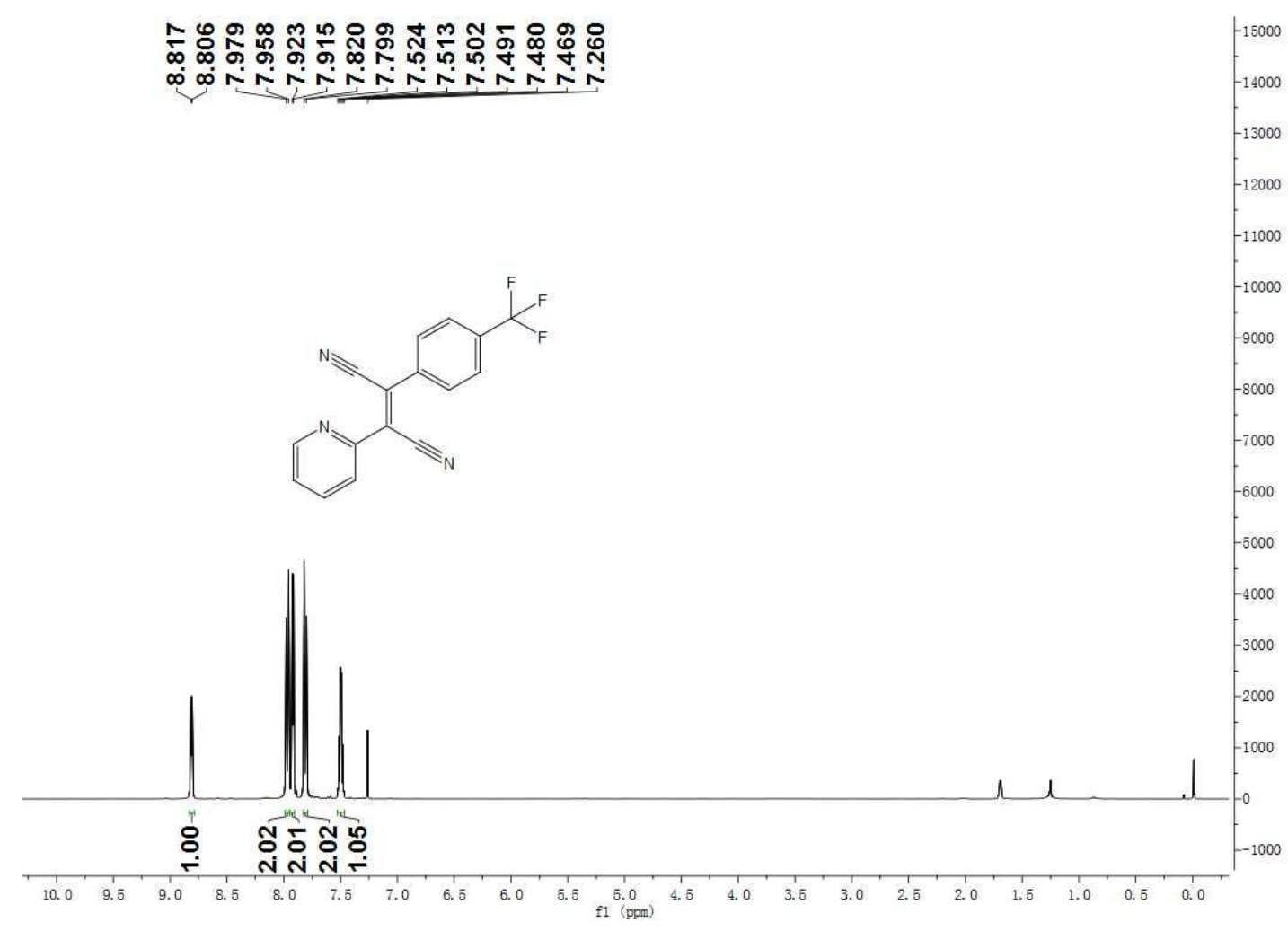

Figure S21. ${ }^{1} \mathrm{H}$ NMR spectrum of $3 \mathrm{k}\left(400 \mathrm{MHz}, \mathrm{CDCl}_{3}\right)$

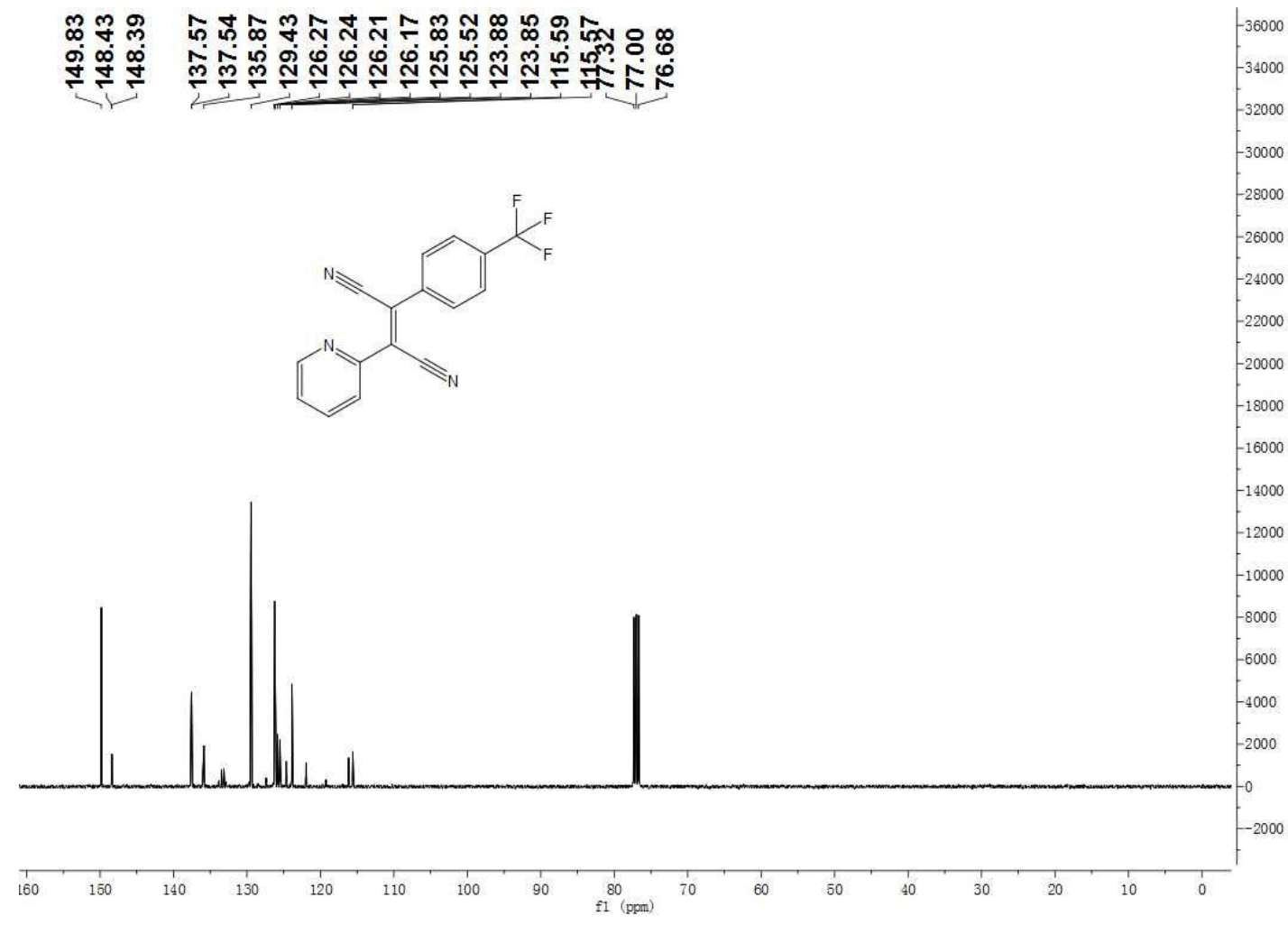

Figure S22. ${ }^{13} \mathrm{C}\left\{{ }^{1} \mathrm{H}\right\}$ NMR spectrum of $3 \mathrm{k}\left(100 \mathrm{MHz}, \mathrm{CDCl}_{3}\right)$ 


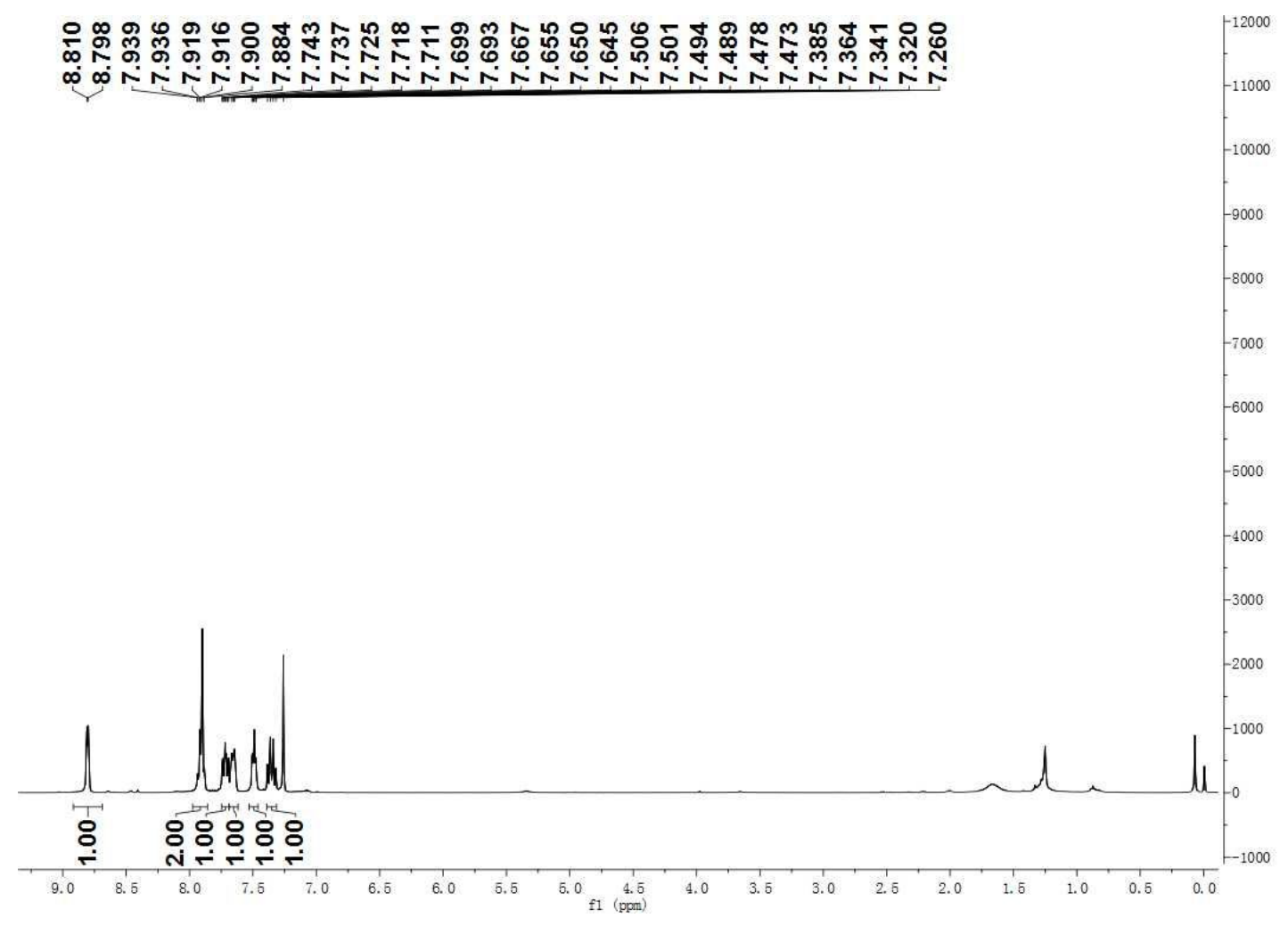

Figure S23. ${ }^{1} \mathrm{H}$ NMR spectrum of 31 (400 $\left.\mathrm{MHz}, \mathrm{CDCl}_{3}\right)$

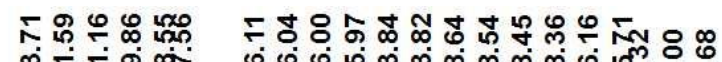

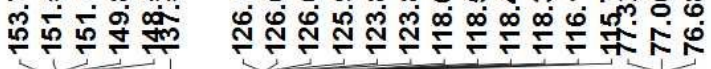

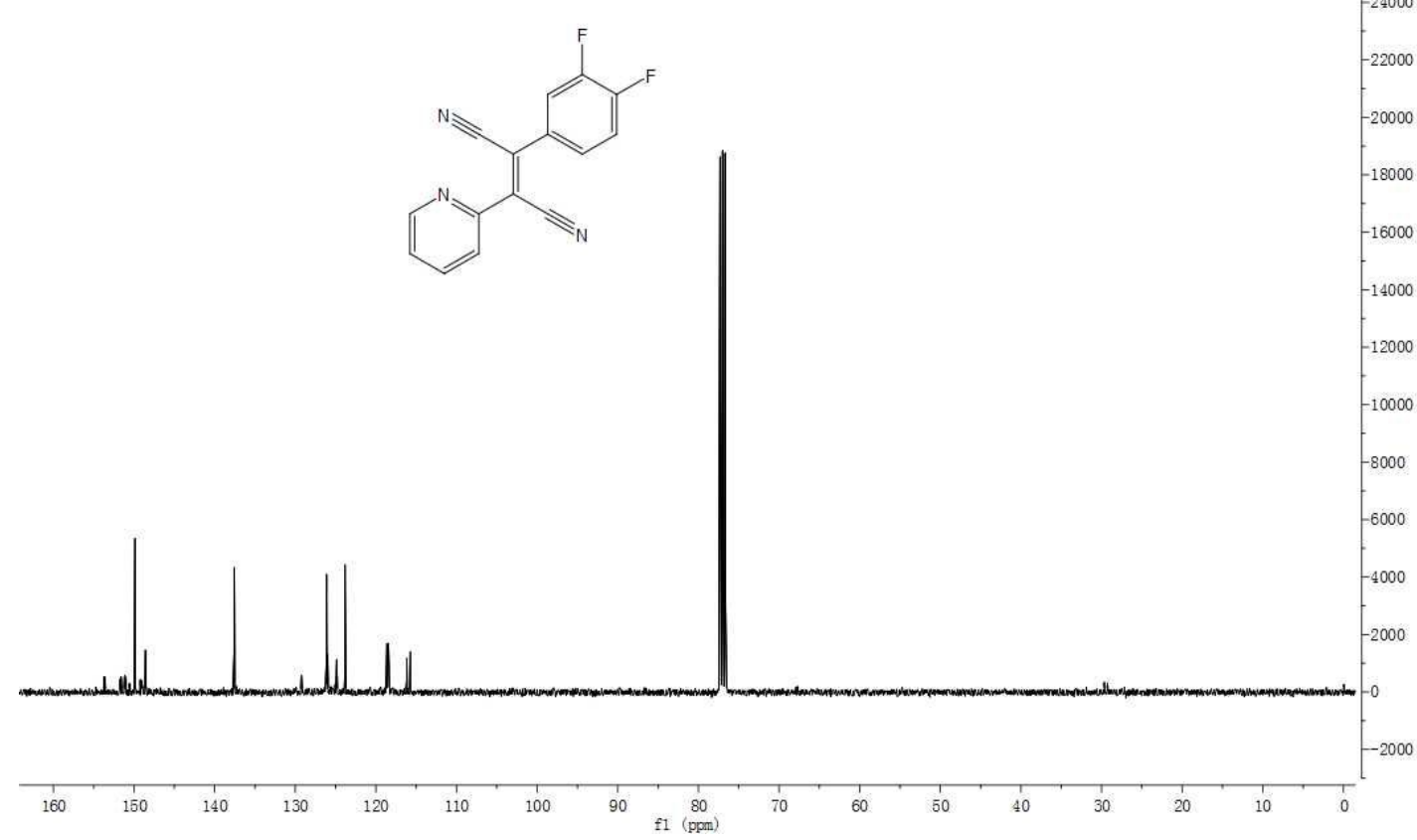

Figure S24. ${ }^{13} \mathrm{C}\left\{{ }^{1} \mathrm{H}\right\}$ NMR spectrum of $31\left(100 \mathrm{MHz}, \mathrm{CDCl}_{3}\right)$ 


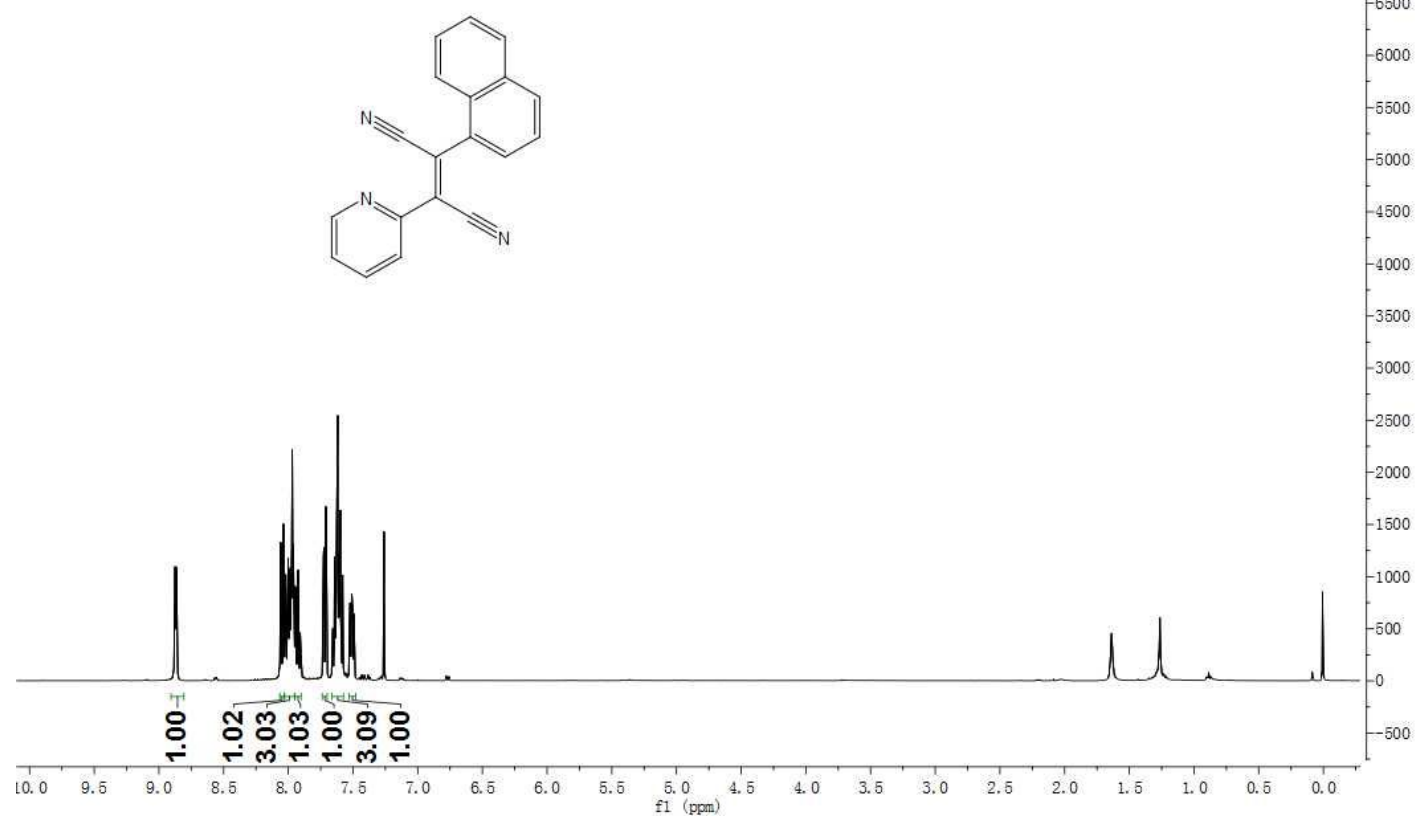

Figure S25. ${ }^{1} \mathrm{H}$ NMR spectrum of $3 \mathrm{~m}\left(400 \mathrm{MHz} \mathrm{CDCl}_{3}\right)$

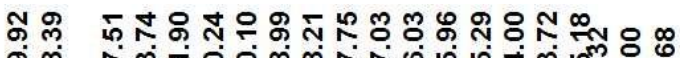

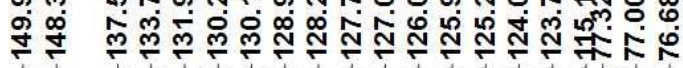

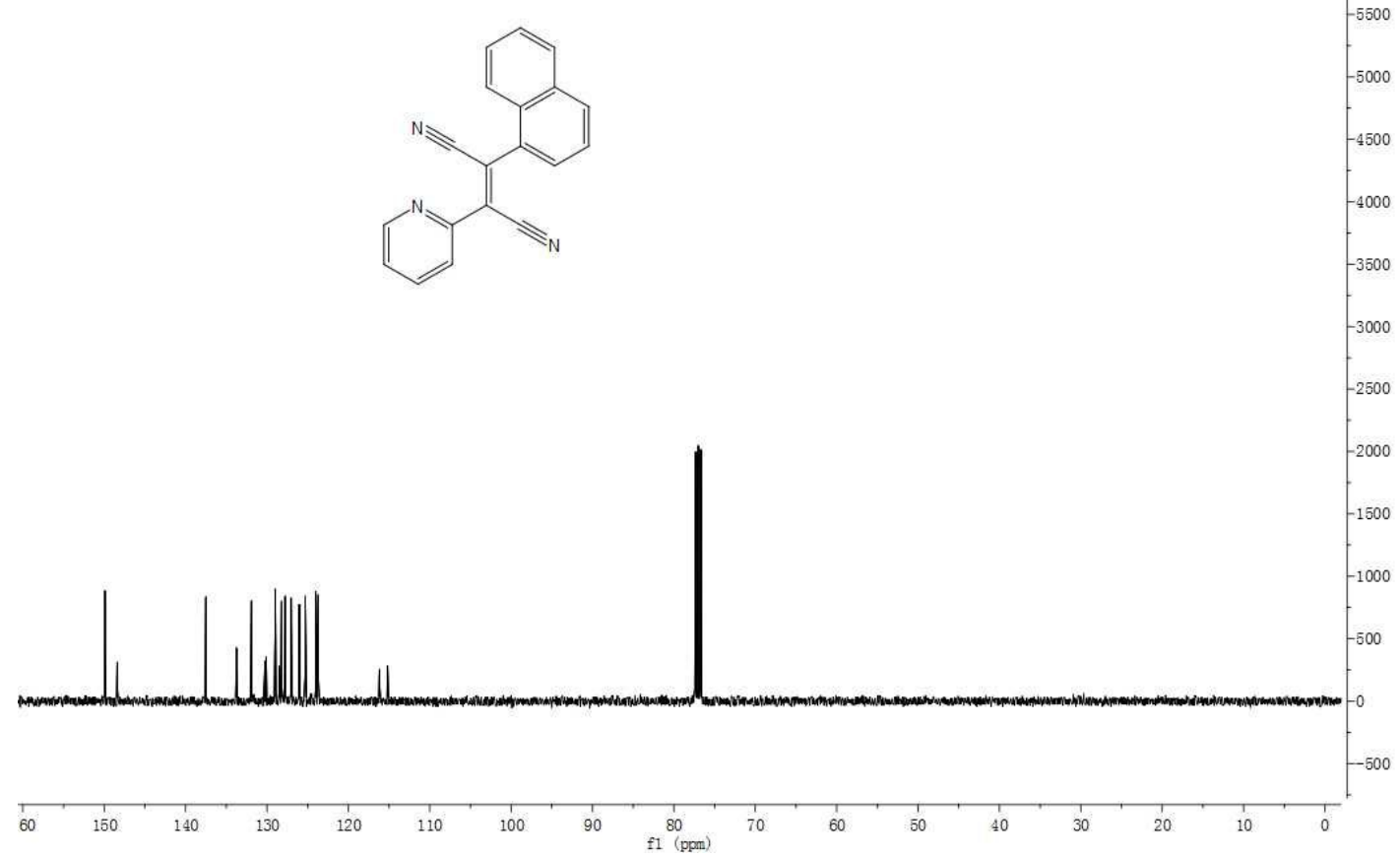

Figure S26. ${ }^{13} \mathrm{C}\left\{{ }^{1} \mathrm{H}\right\}$ NMR spectrum of $3 \mathrm{~m}\left(100 \mathrm{MHz}, \mathrm{CDCl}_{3}\right)$ 


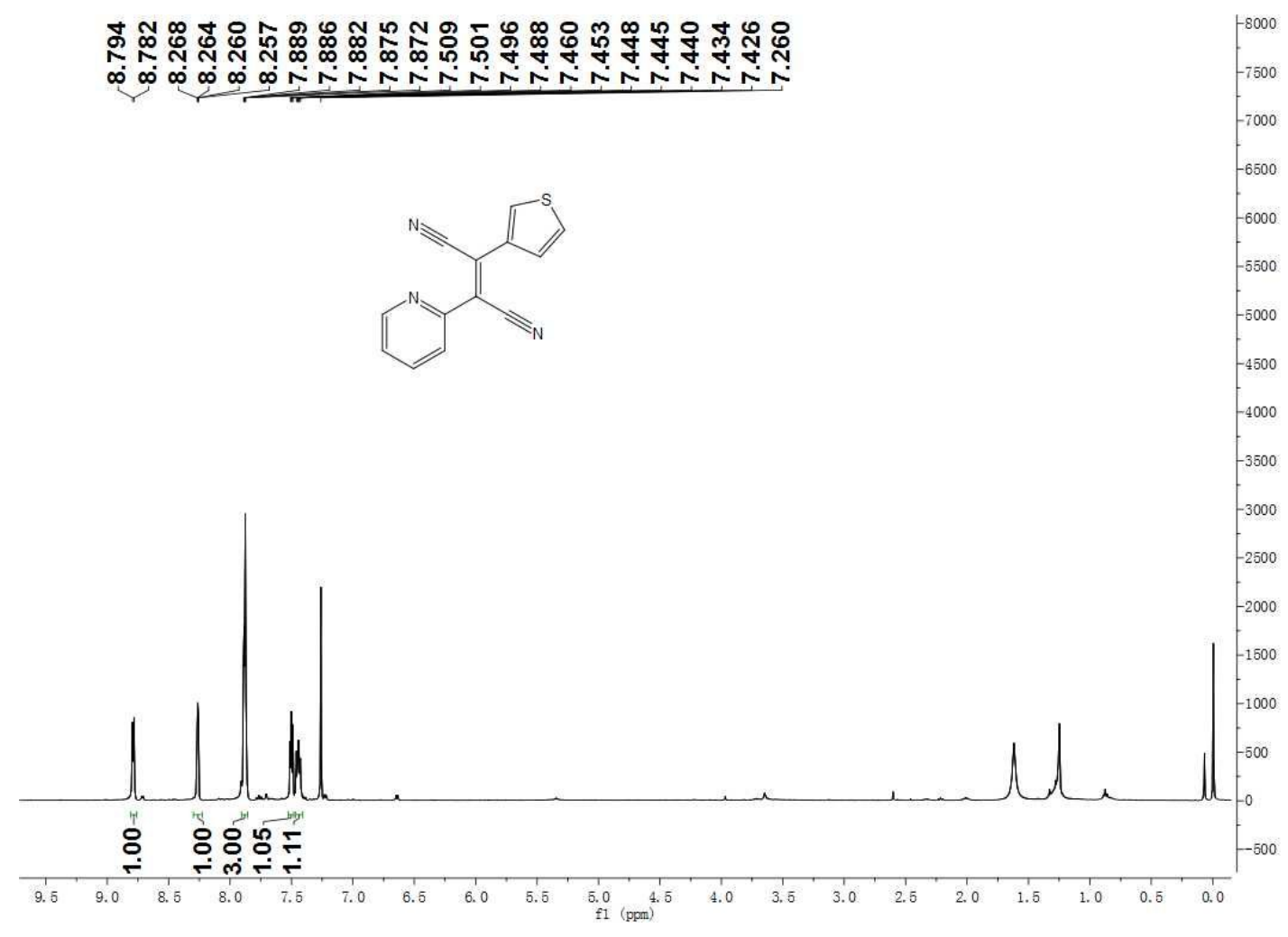

Figure S27. ${ }^{1} \mathrm{H}$ NMR spectrum of $3 \mathrm{n}\left(400 \mathrm{MHz}, \mathrm{CDCl}_{3}\right)$

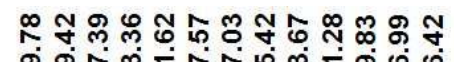

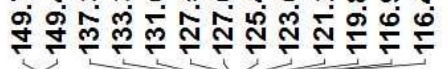

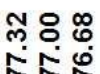

实实
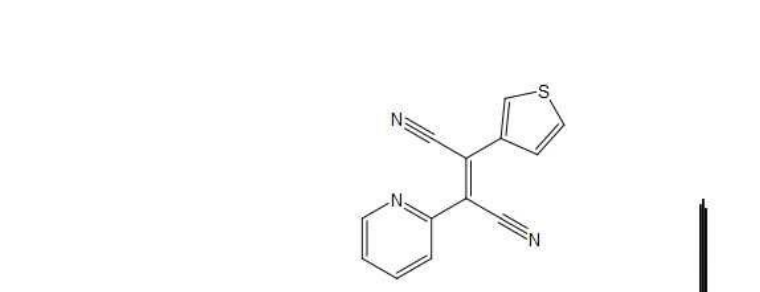


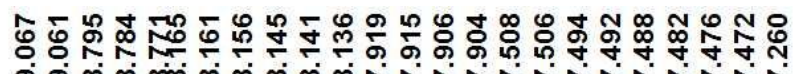

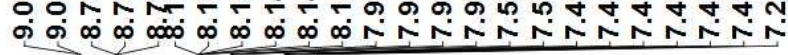

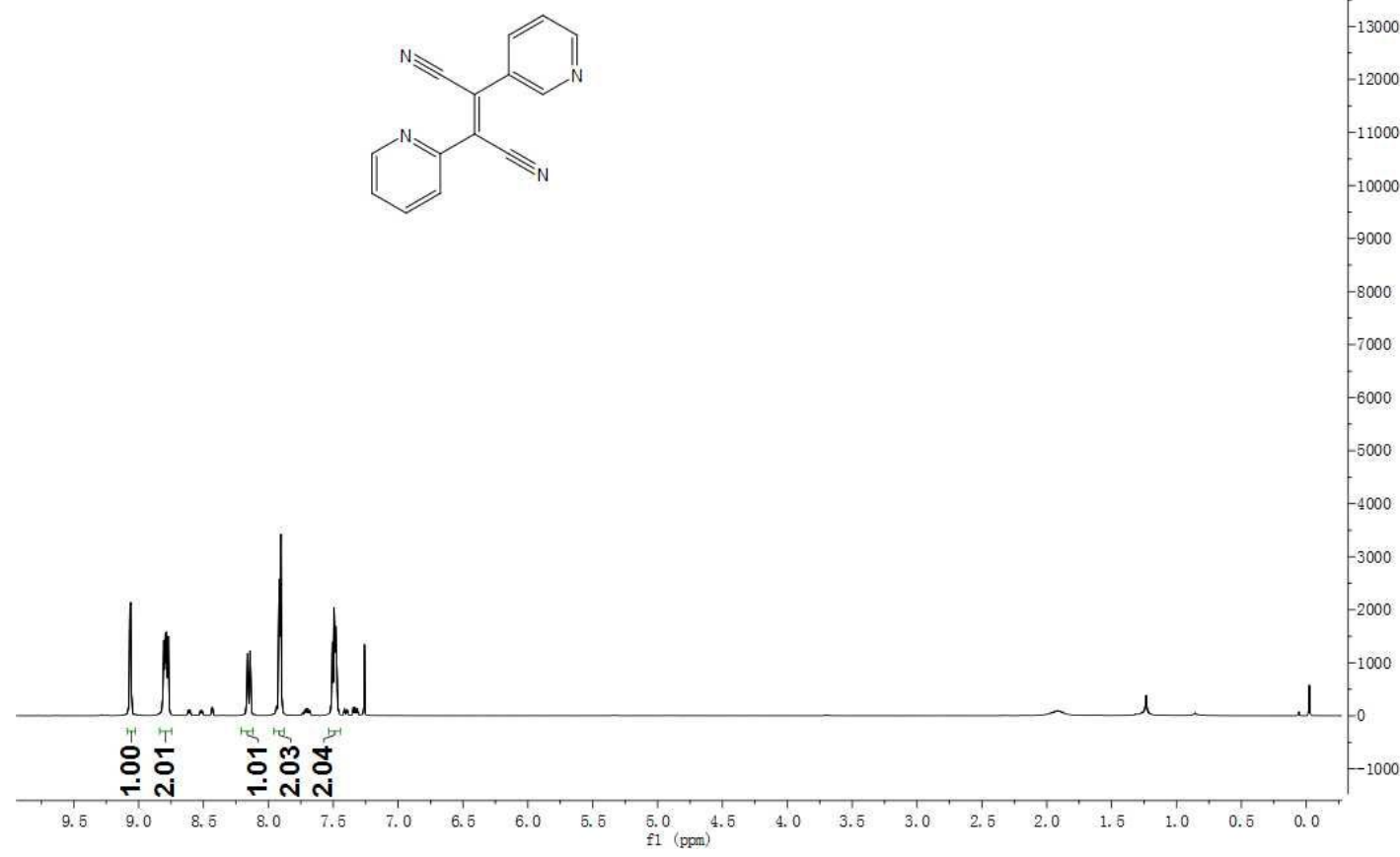

Figure S29. ${ }^{1} \mathrm{H}$ NMR spectrum of $30\left(400 \mathrm{MHz}, \mathrm{CDCl}_{3}\right)$

ஸุ

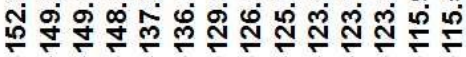

ก้ 8 \%

FN

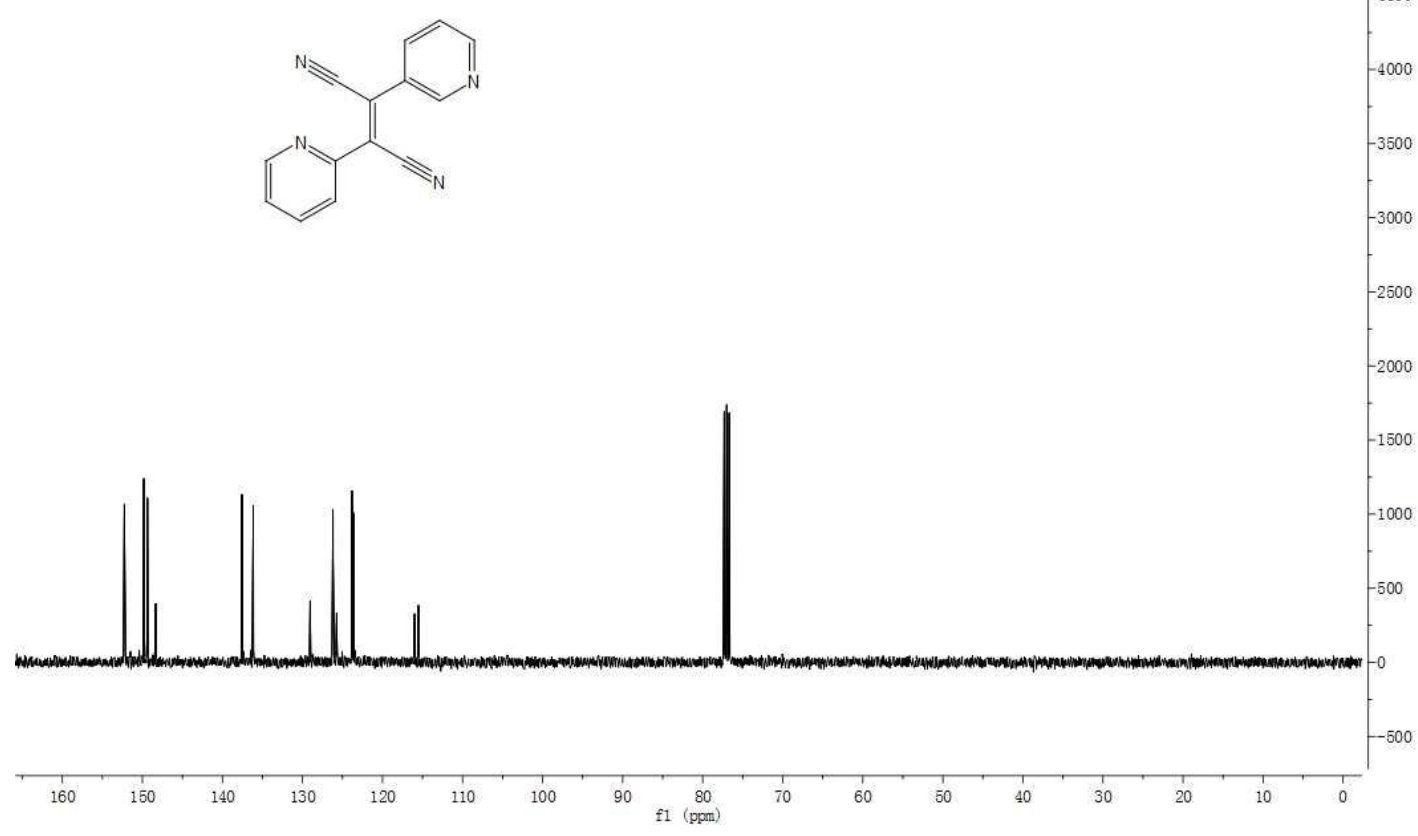

Figure S30. ${ }^{13} \mathrm{C}\left\{{ }^{1} \mathrm{H}\right\}$ NMR spectrum of $30\left(100 \mathrm{MHz}, \mathrm{CDCl}_{3}\right)$ 
获

$\infty \infty N N N N N N N N$

$\stackrel{\text { 守 }}{\text { i }}$

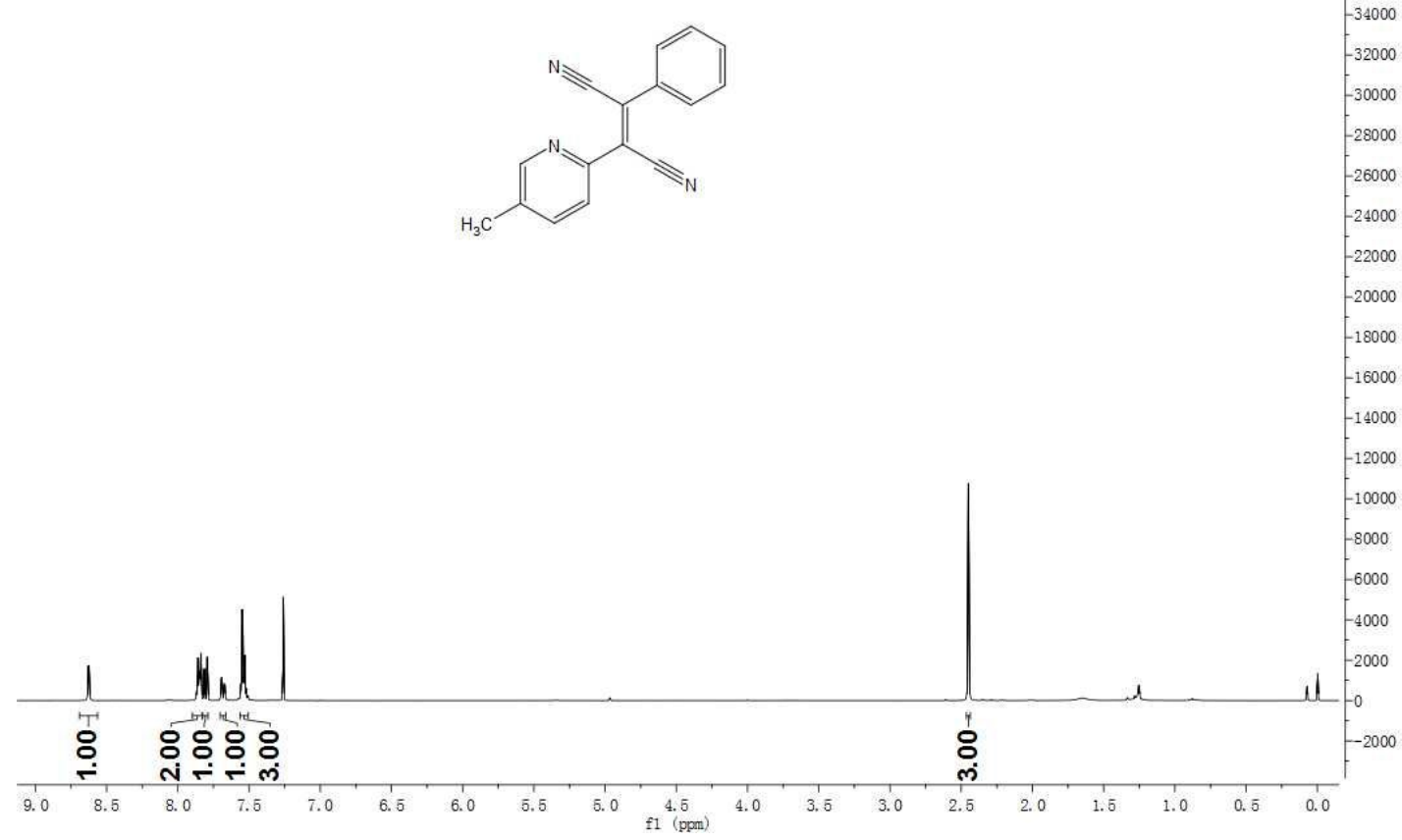

Figure S31. ${ }^{1} \mathrm{H}$ NMR spectrum of $3 p\left(400 \mathrm{MHz}, \mathrm{CDCl}_{3}\right)$

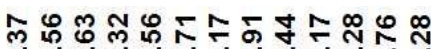

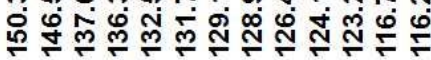
กัలి

송

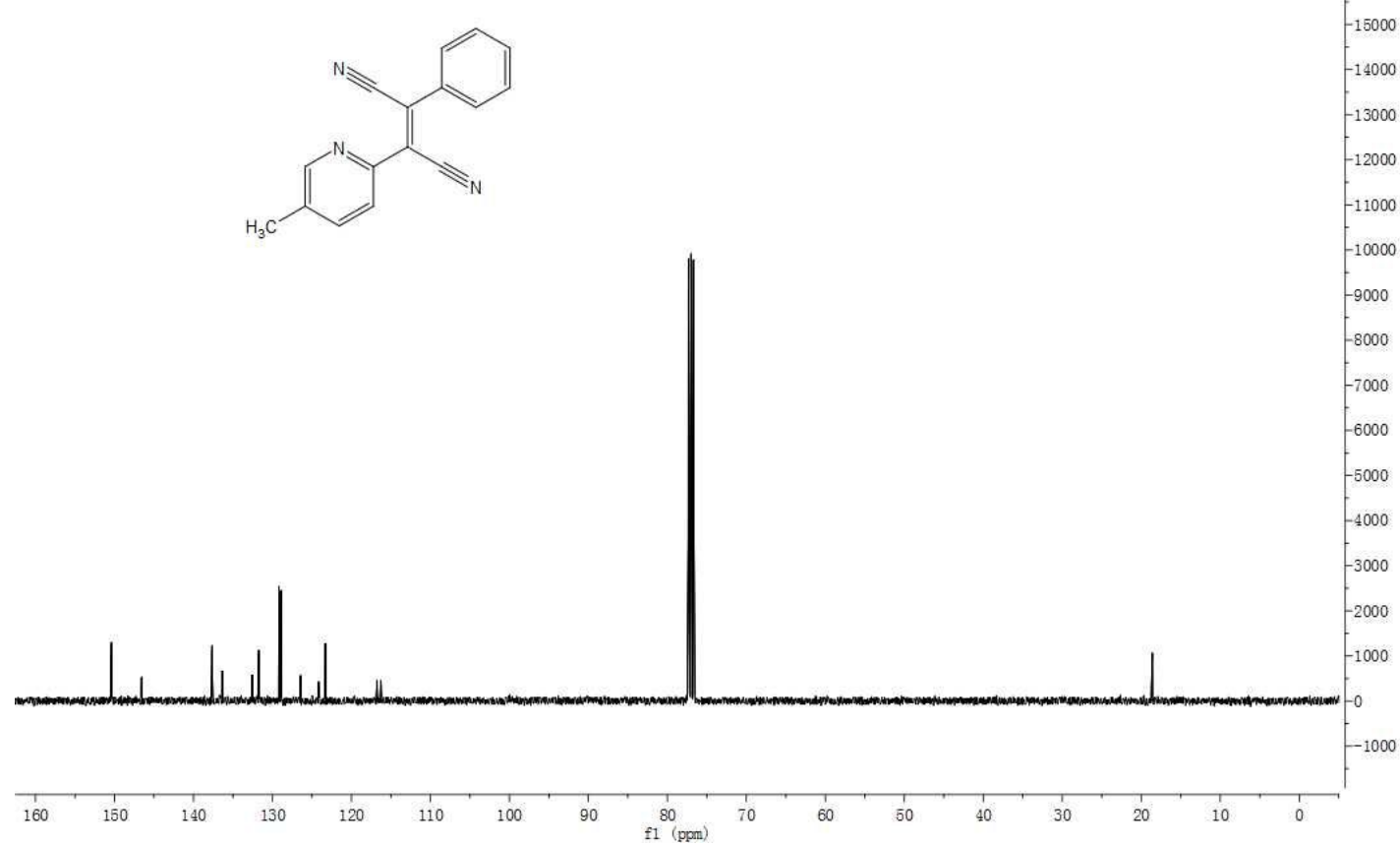

Figure S32. ${ }^{13} \mathrm{C}\left\{{ }^{1} \mathrm{H}\right\}$ NMR spectrum of $3 p\left(100 \mathrm{MHz}, \mathrm{CDCl}_{3}\right)$ 


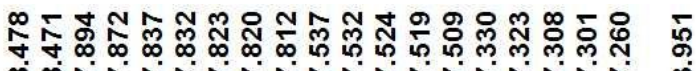

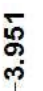

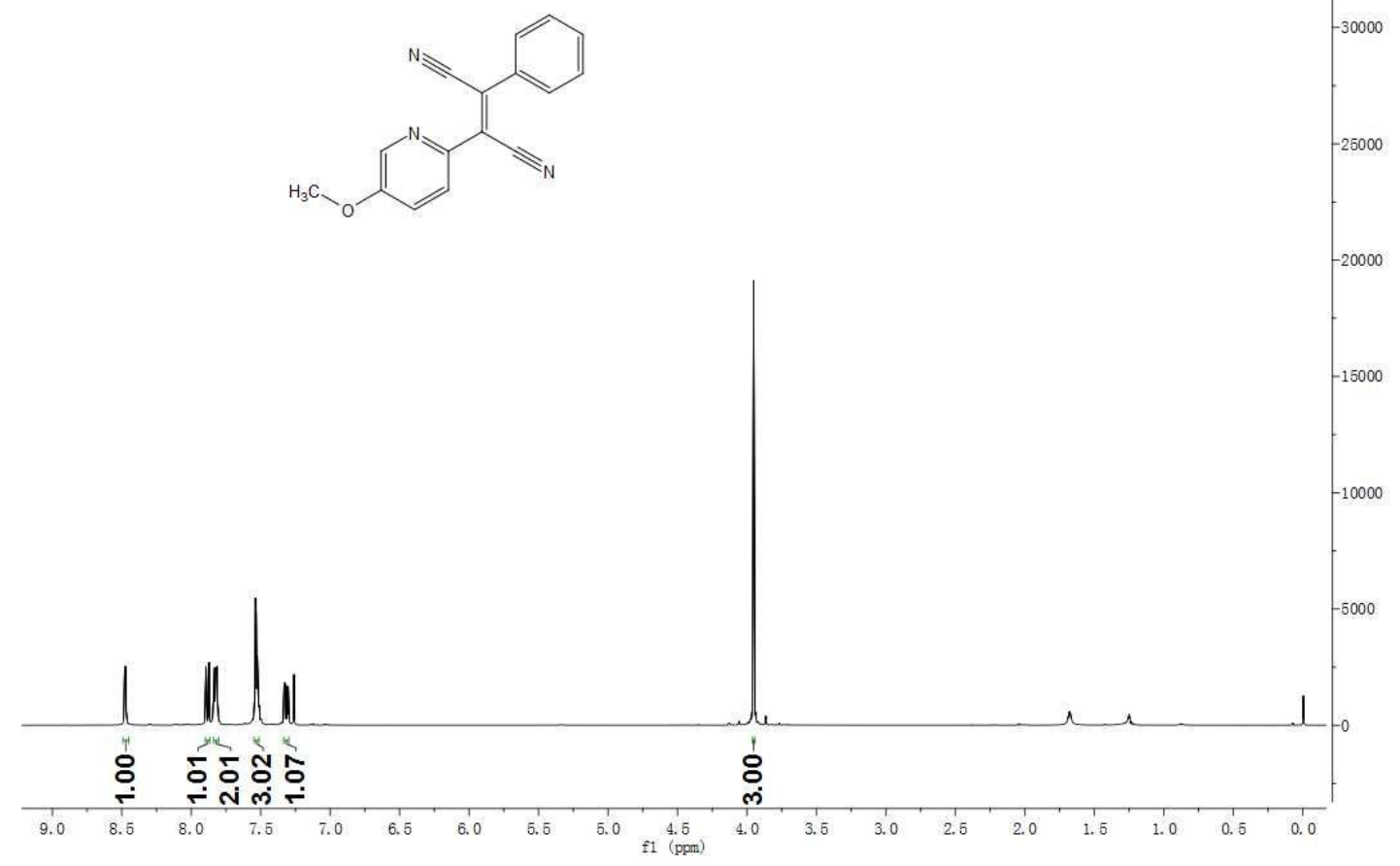

Figure S33. ${ }^{1} \mathrm{H}$ NMR spectrum of $3 q\left(400 \mathrm{MHz}, \mathrm{CDCl}_{3}\right)$

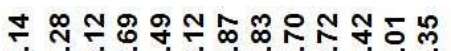

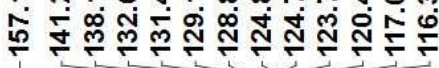

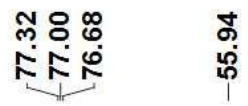

$-7000$

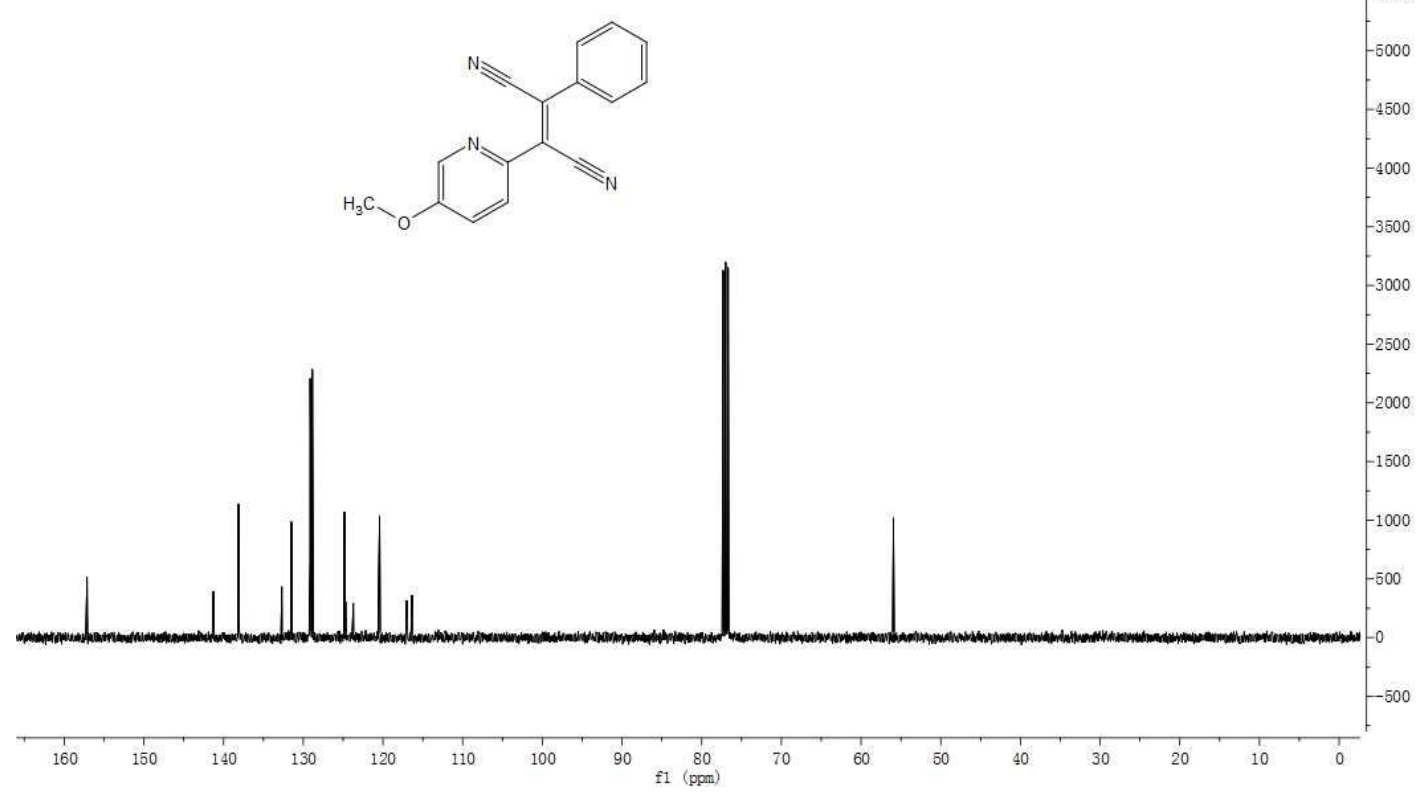

$-5500$

Figure S34. ${ }^{13} \mathrm{C}\left\{{ }^{1} \mathrm{H}\right\}$ NMR spectrum of $3 q\left(100 \mathrm{MHz}, \mathrm{CDCl}_{3}\right)$ 


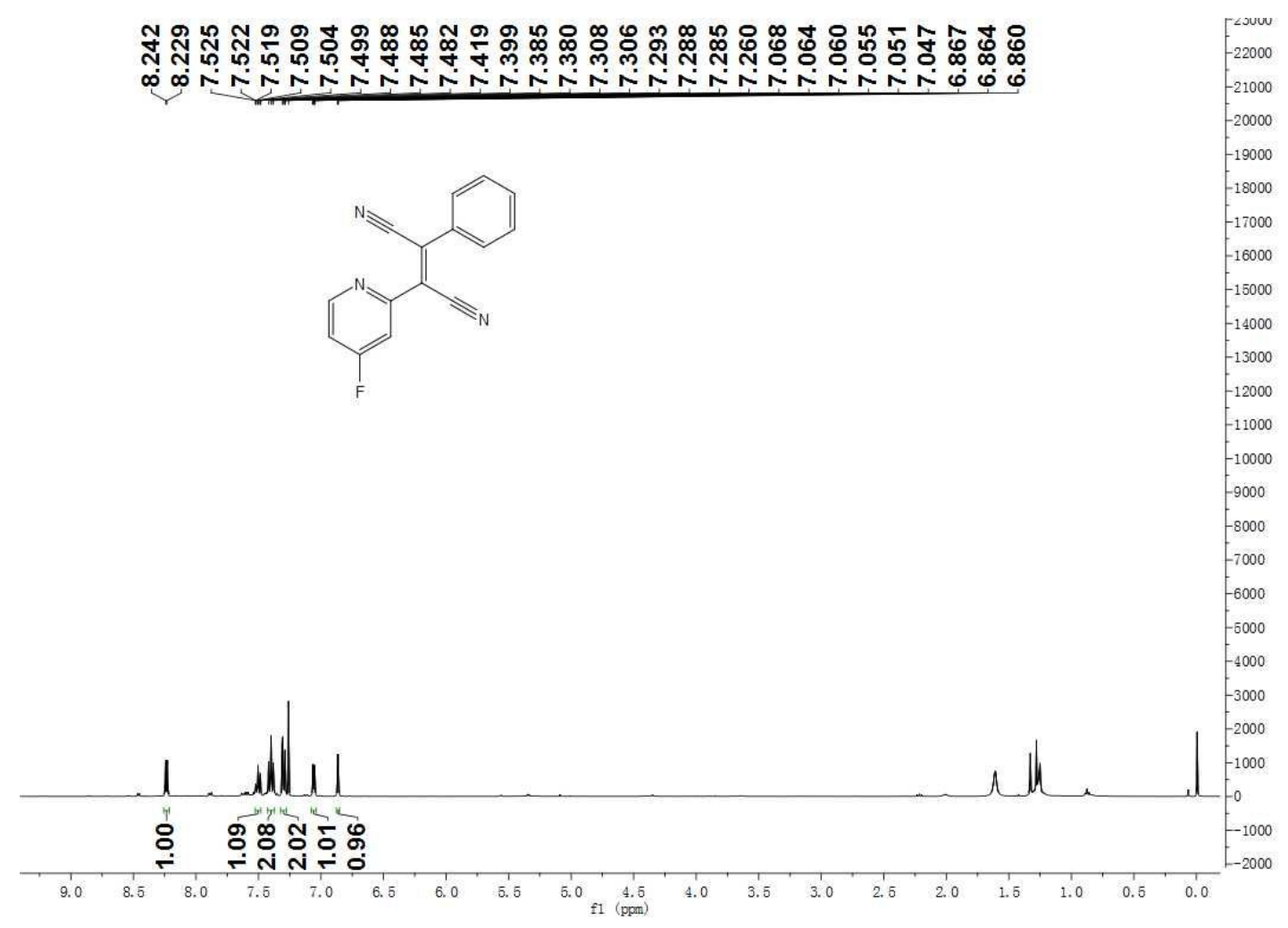

Figure S35. ${ }^{1} \mathrm{H}$ NMR spectrum of $3 \mathrm{r}\left(400 \mathrm{MHz}, \mathrm{CDCl}_{3}\right)$

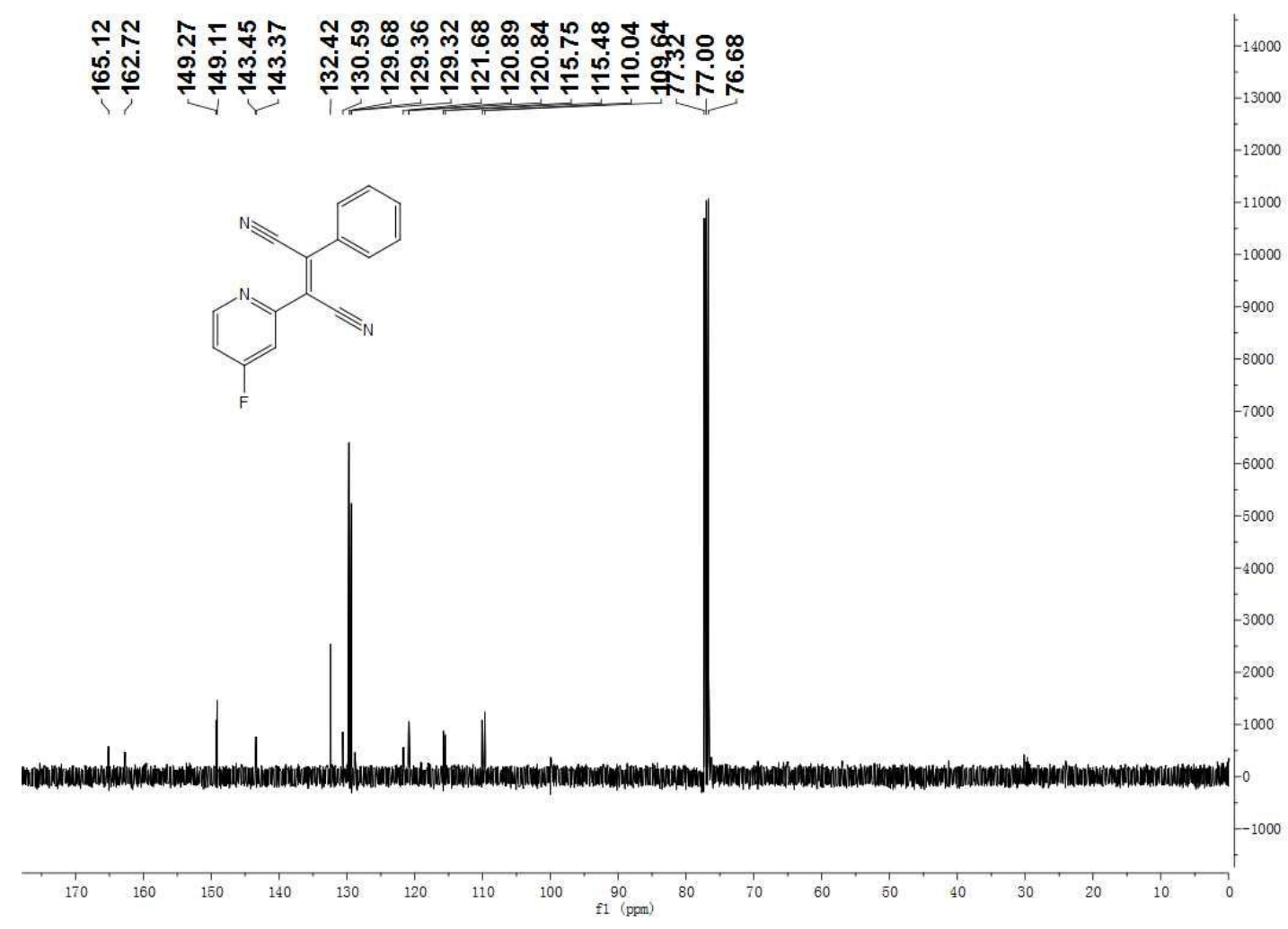

Figure S36. ${ }^{13} \mathrm{C}\left\{{ }^{1} \mathrm{H}\right\}$ NMR spectrum of 3 r (100 $\left.\mathrm{MHz}, \mathrm{CDCl}_{3}\right)$ 


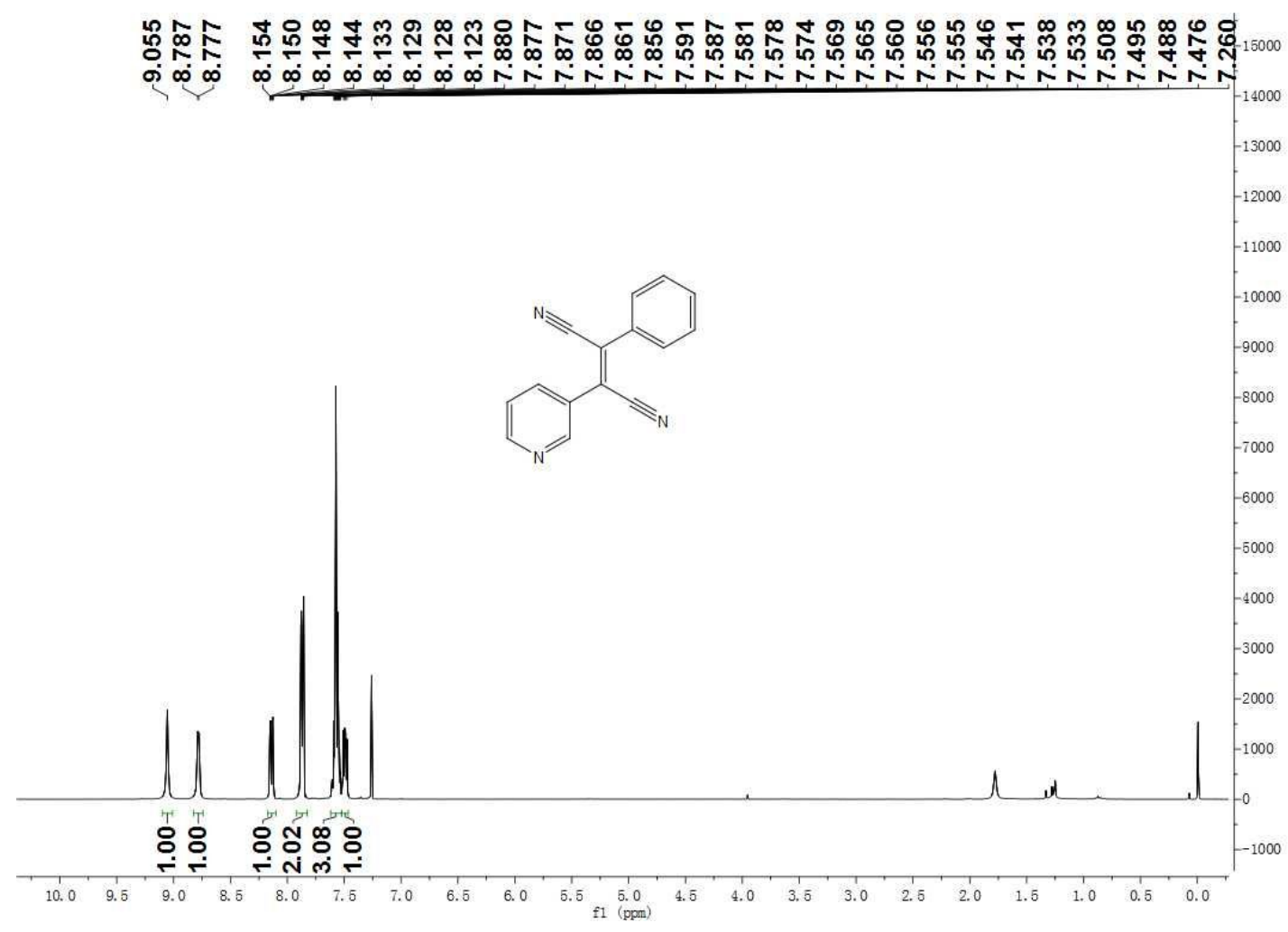

Figure S37. ${ }^{1} \mathrm{H}$ NMR spectrum of $3 s\left(400 \mathrm{MHz}, \mathrm{CDCl}_{3}\right)$

กำ

ํำ 守

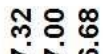

R̂́

30000

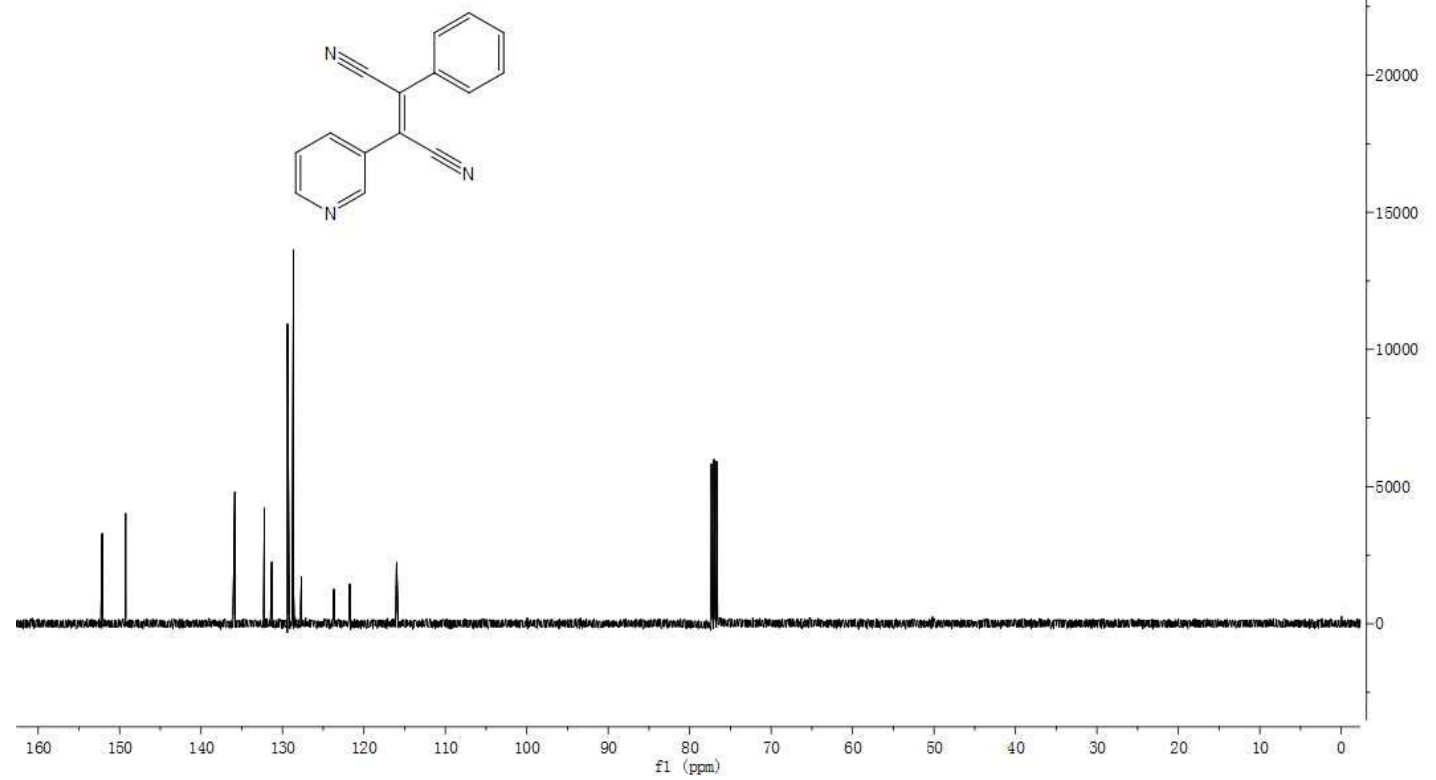

Figure S38. ${ }^{13} \mathrm{C}\left\{{ }^{1} \mathrm{H}\right\}$ NMR spectrum of $3 \mathrm{~s}\left(100 \mathrm{MHz}, \mathrm{CDCl}_{3}\right)$ 


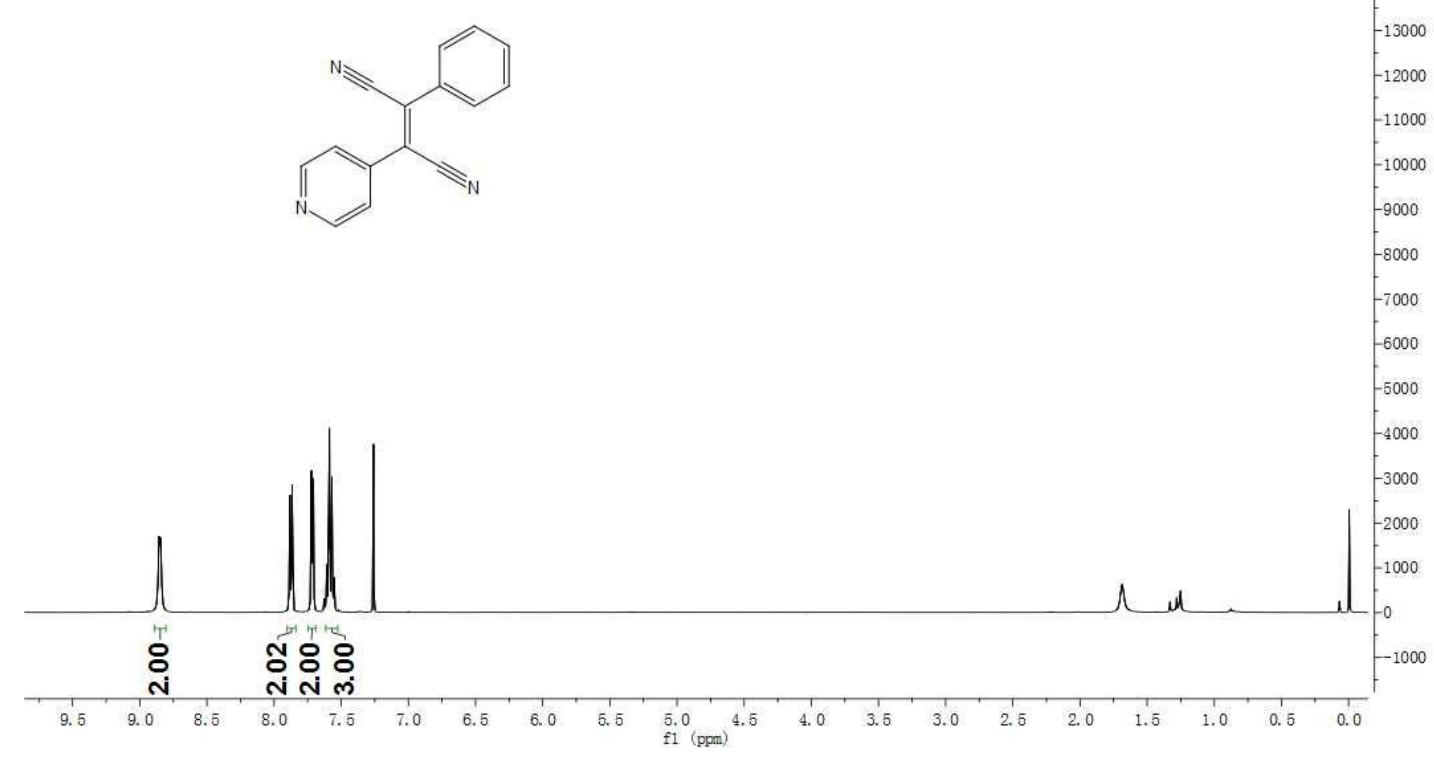

Figure S39. ${ }^{1} \mathrm{H}$ NMR spectrum of $3 \mathrm{t}\left(400 \mathrm{MHz}, \mathrm{CDCl}_{3}\right)$

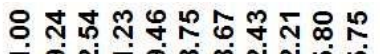

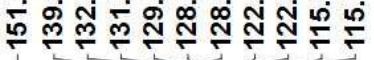

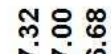

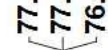

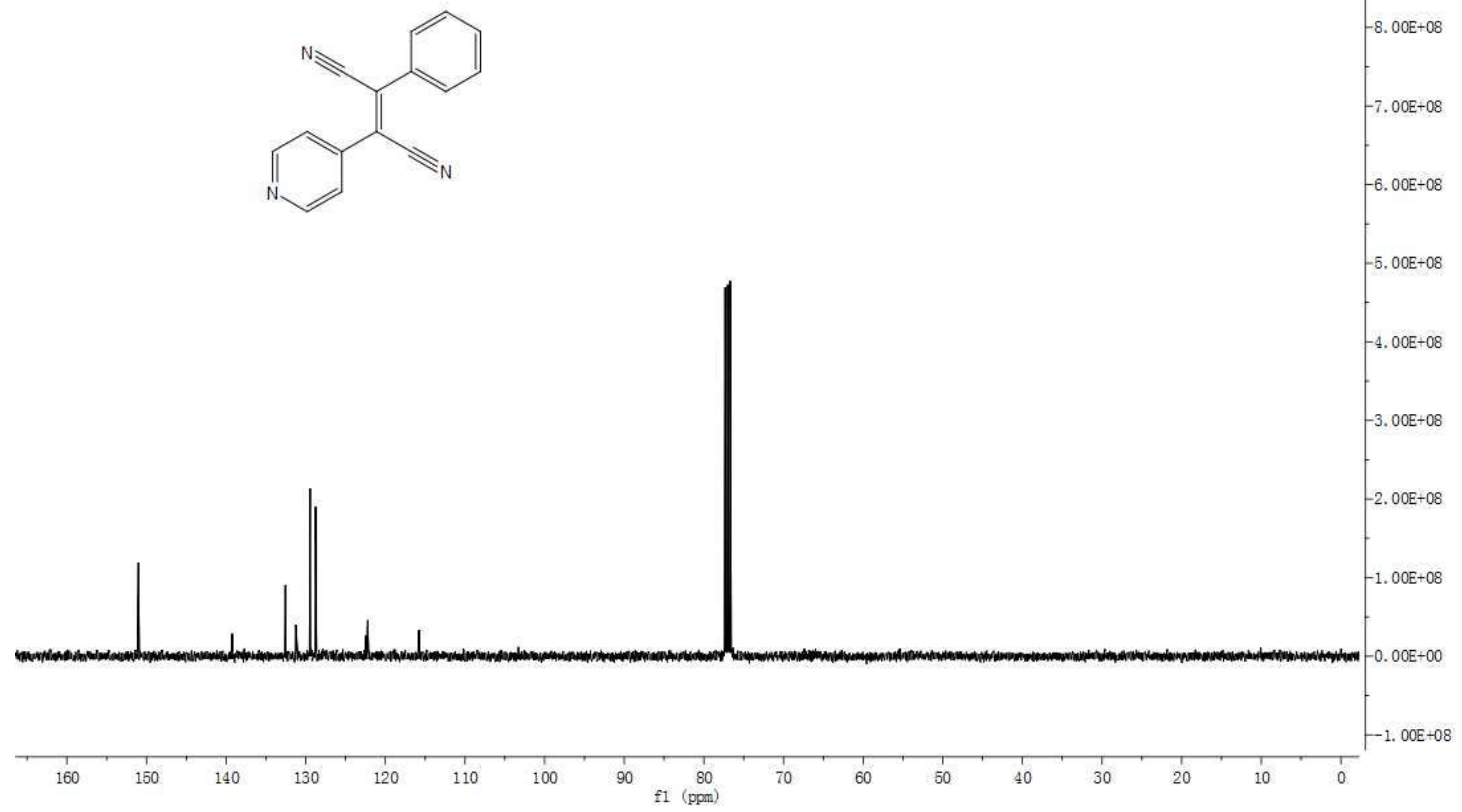

Figure S40. ${ }^{13} \mathrm{C}\left\{{ }^{1} \mathrm{H}\right\}$ NMR spectrum of $3 \mathrm{t}\left(100 \mathrm{MHz}, \mathrm{CDCl}_{3}\right)$ 


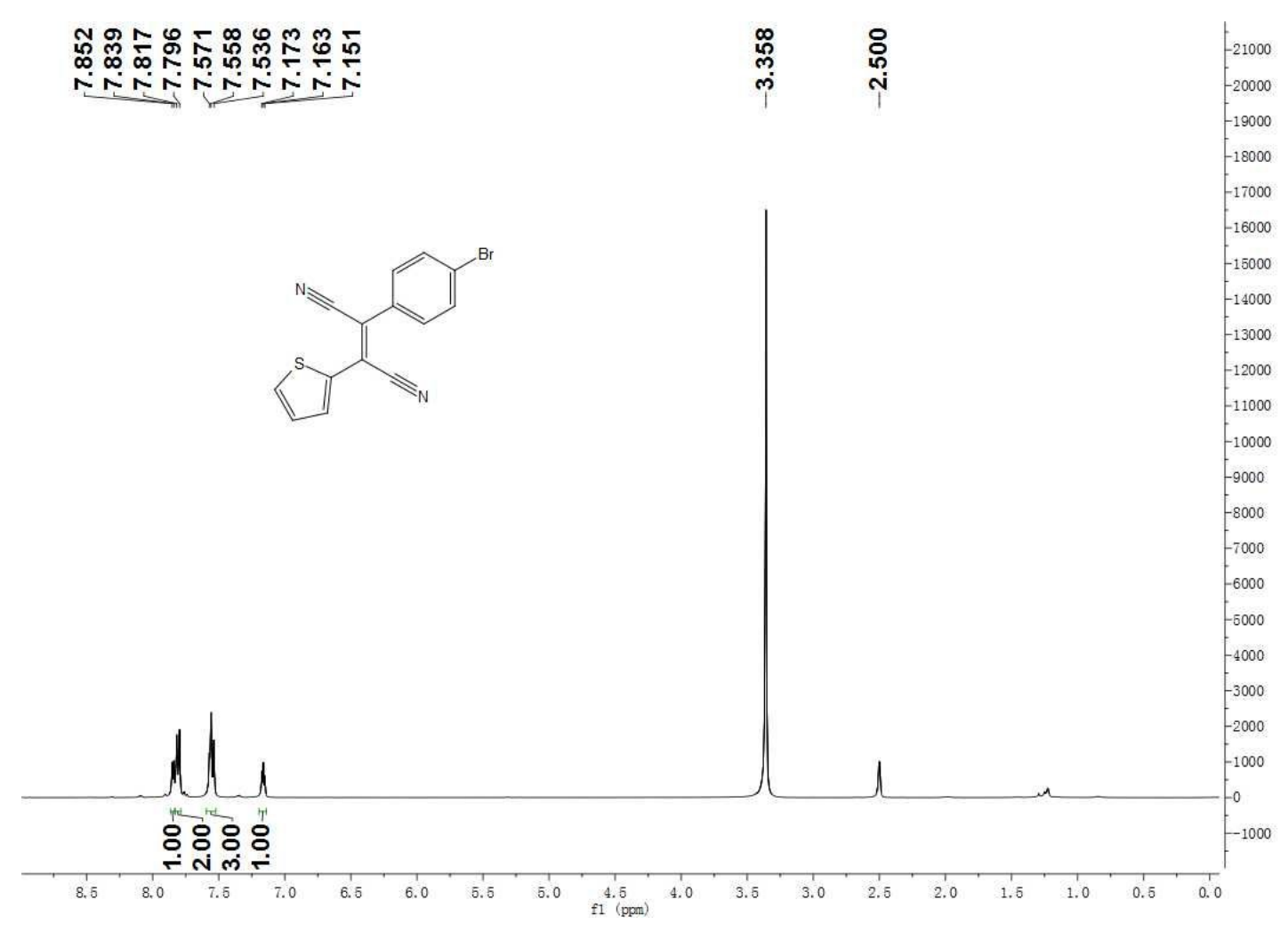

Figure S41. ${ }^{1} \mathrm{H}$ NMR spectrum of $3 \mathrm{u}(400 \mathrm{MHz}$, DMSO-d6)

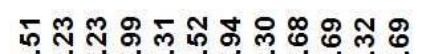

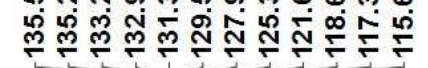

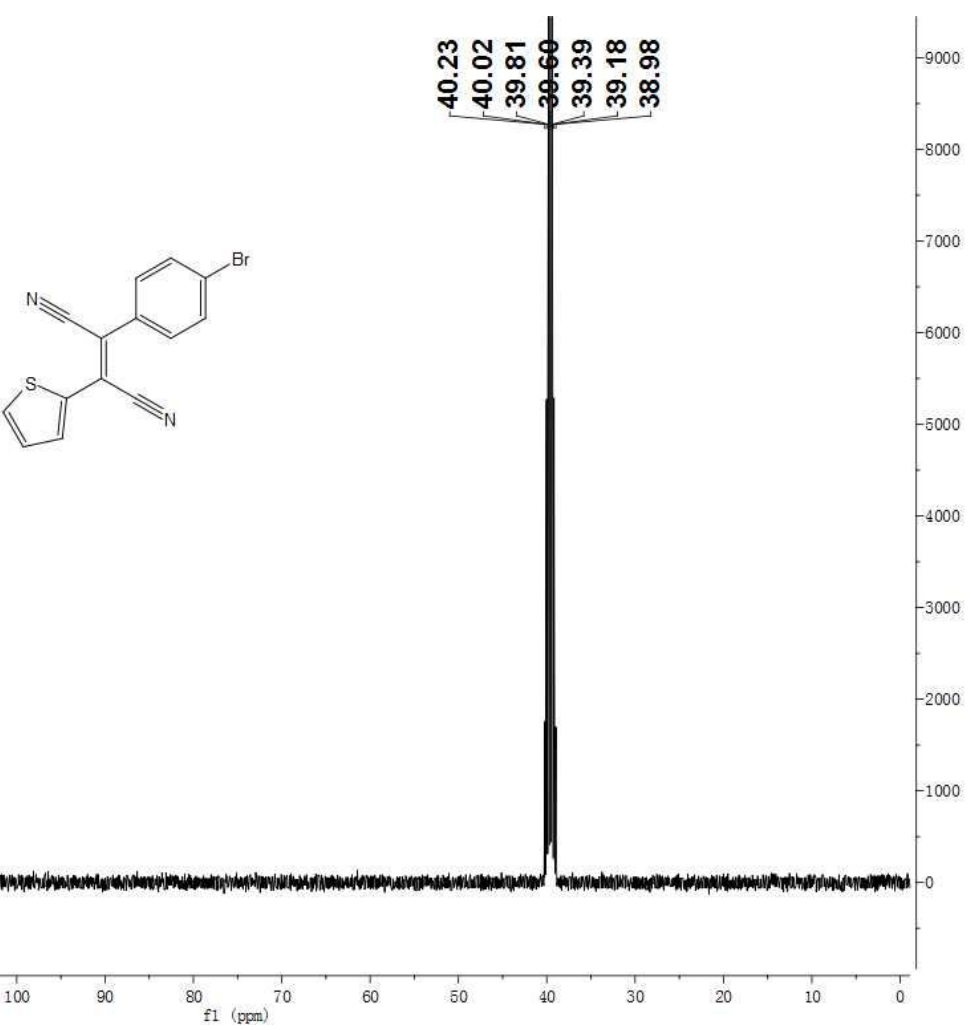

Figure S42. ${ }^{13} \mathrm{C}\left\{{ }^{1} \mathrm{H}\right\}$ NMR spectrum of $3 \mathrm{u}(100 \mathrm{MHz}$, DMSO-d6) 


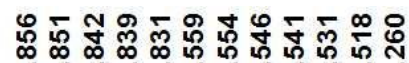

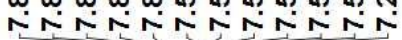

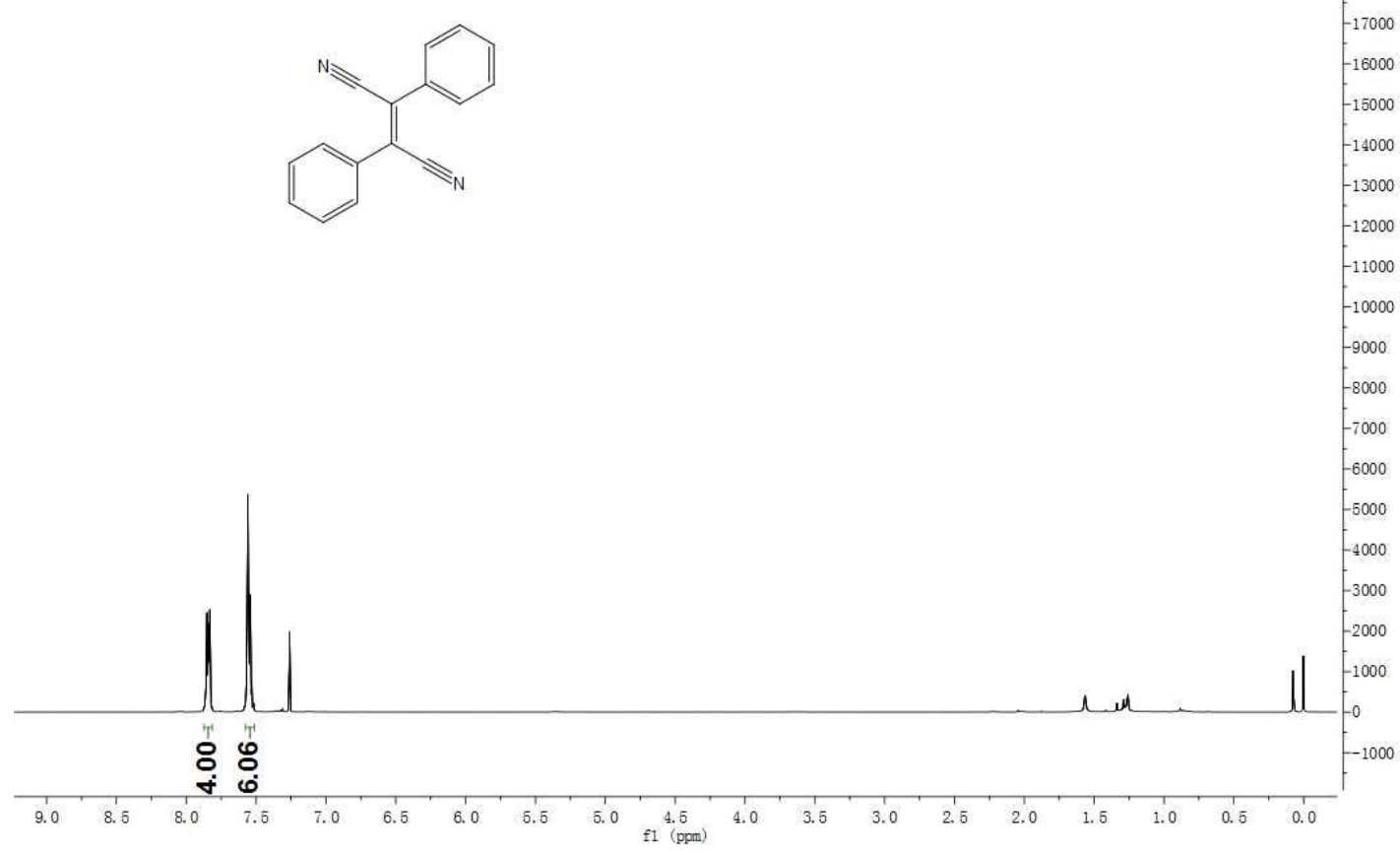

Figure S43. ${ }^{1} \mathrm{H}$ NMR spectrum of 4 a $\left(400 \mathrm{MHz}, \mathrm{CDCl}_{3}\right)$

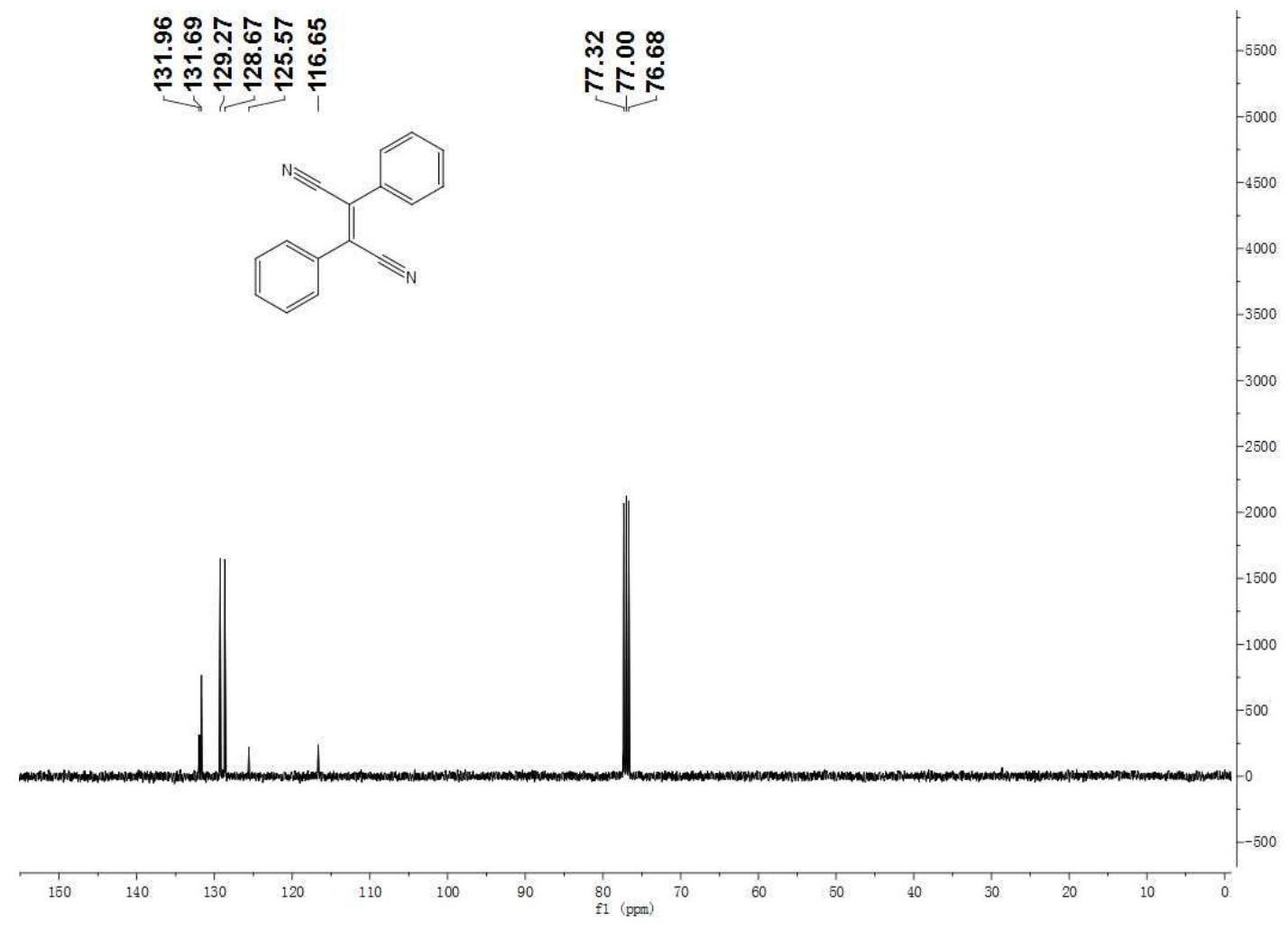

Figure S44. ${ }^{13} \mathrm{C}\left\{{ }^{1} \mathrm{H}\right\}$ NMR spectrum of $4 \mathrm{a}\left(100 \mathrm{MHz}, \mathrm{CDCl}_{3}\right)$ 


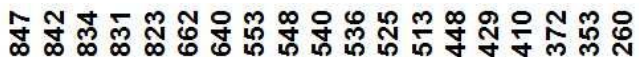

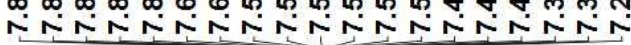

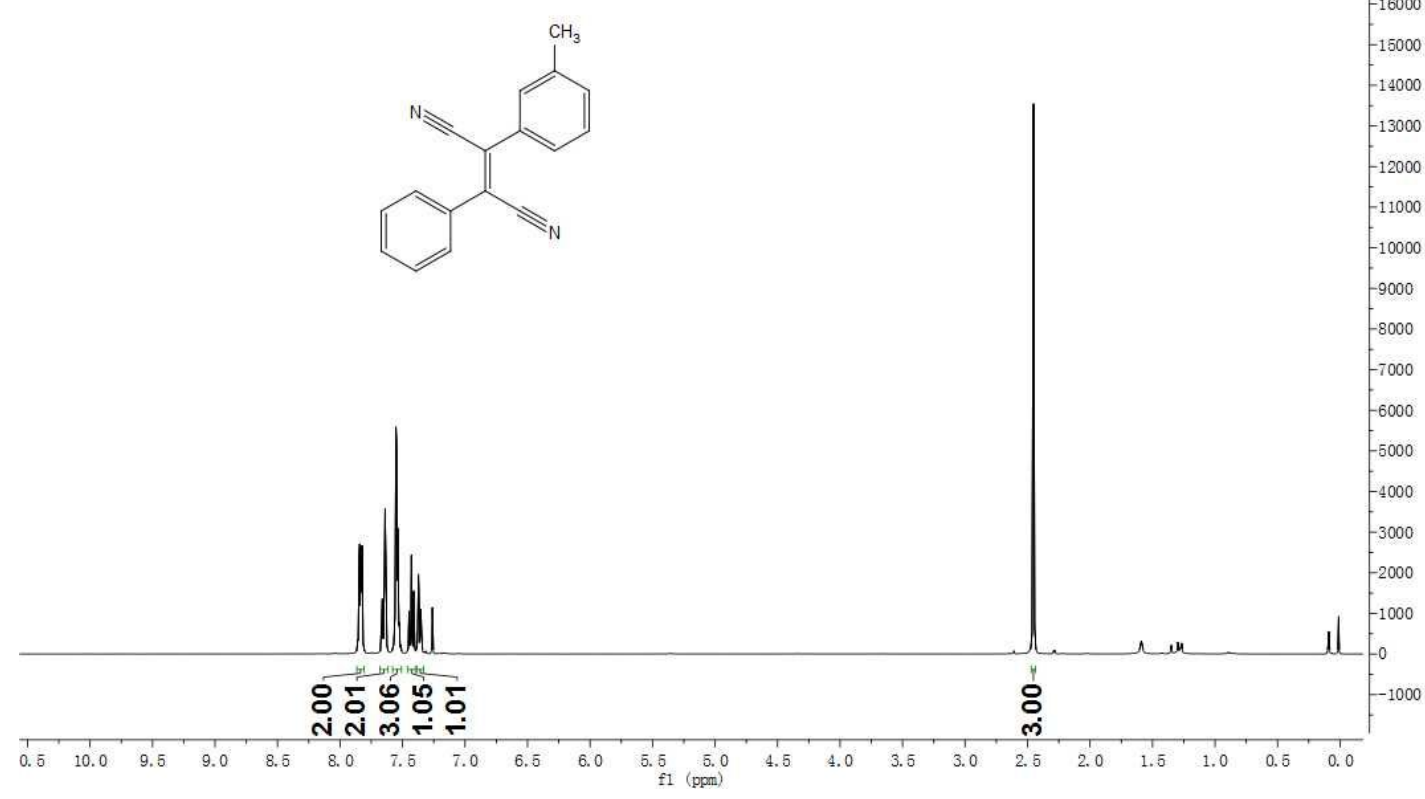

Figure S45. ${ }^{1} \mathrm{H}$ NMR spectrum of $4 \mathrm{~b}\left(400 \mathrm{MHz}, \mathrm{CDCl}_{3}\right)$

Tุ 穴

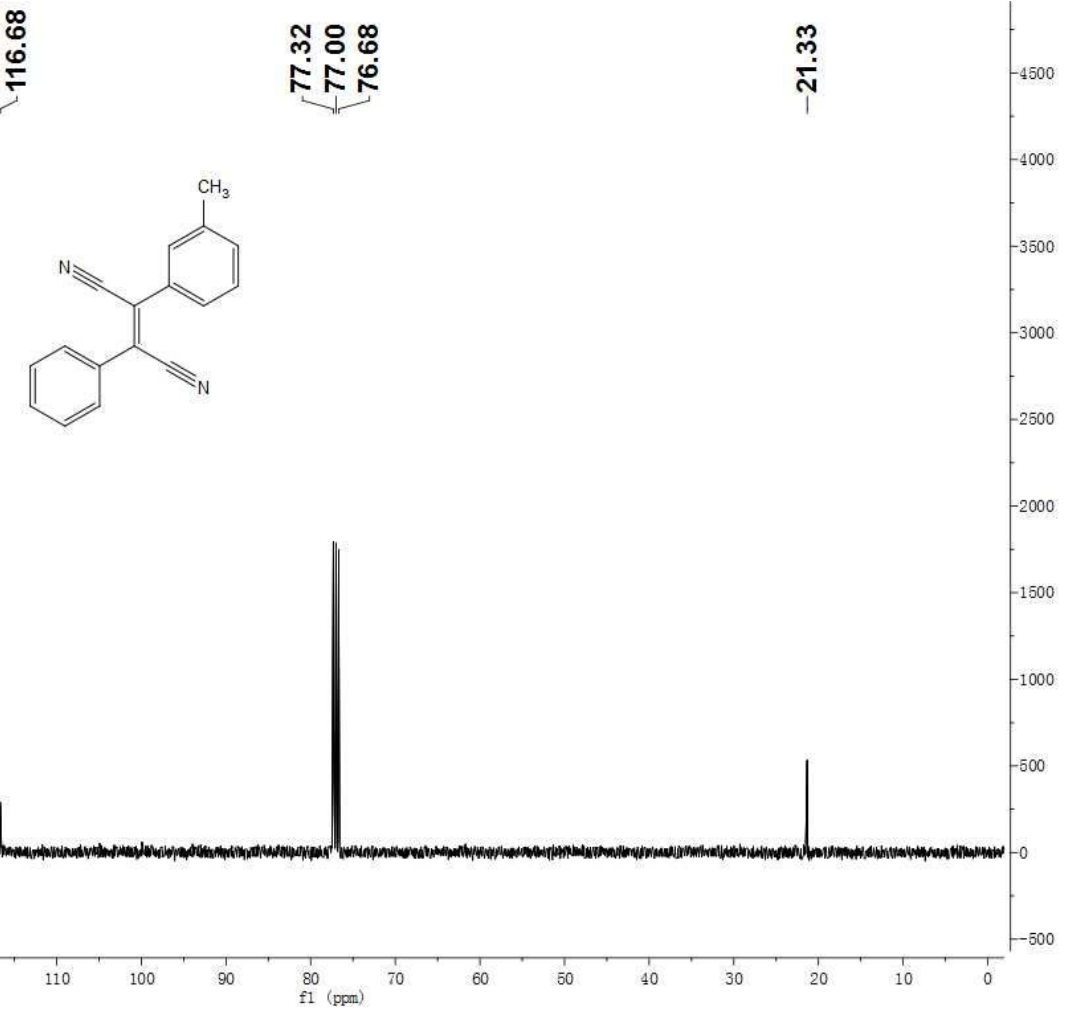

Figure S46. ${ }^{13} \mathrm{C}\left\{{ }^{1} \mathrm{H}\right\}$ NMR spectrum of $4 \mathrm{~b}\left(100 \mathrm{MHz}, \mathrm{CDCl}_{3}\right)$ 


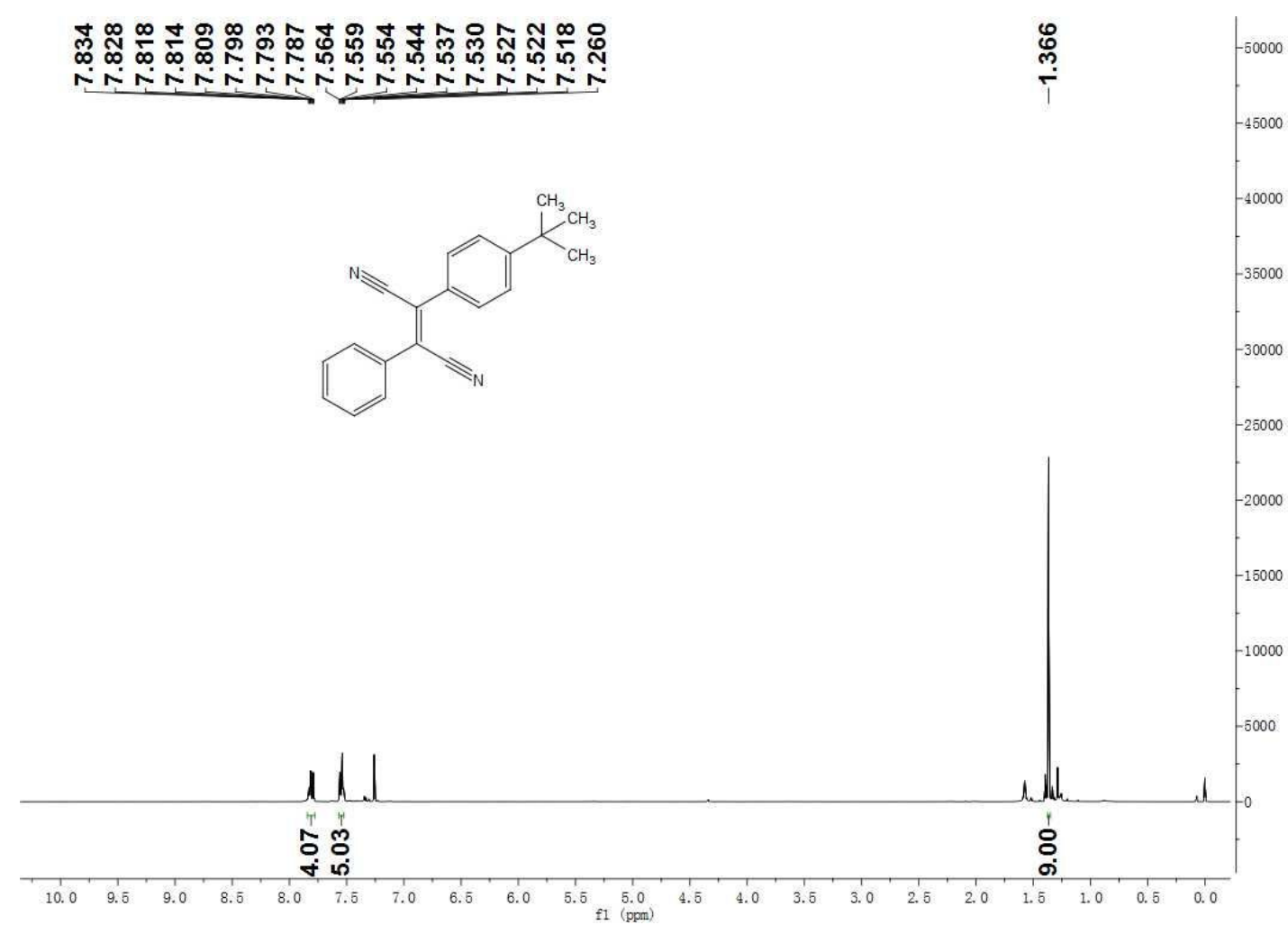

Figure S47. ${ }^{1} \mathrm{H}$ NMR spectrum of $4 \mathrm{c}\left(400 \mathrm{MHz}, \mathrm{CDCl}_{3}\right)$

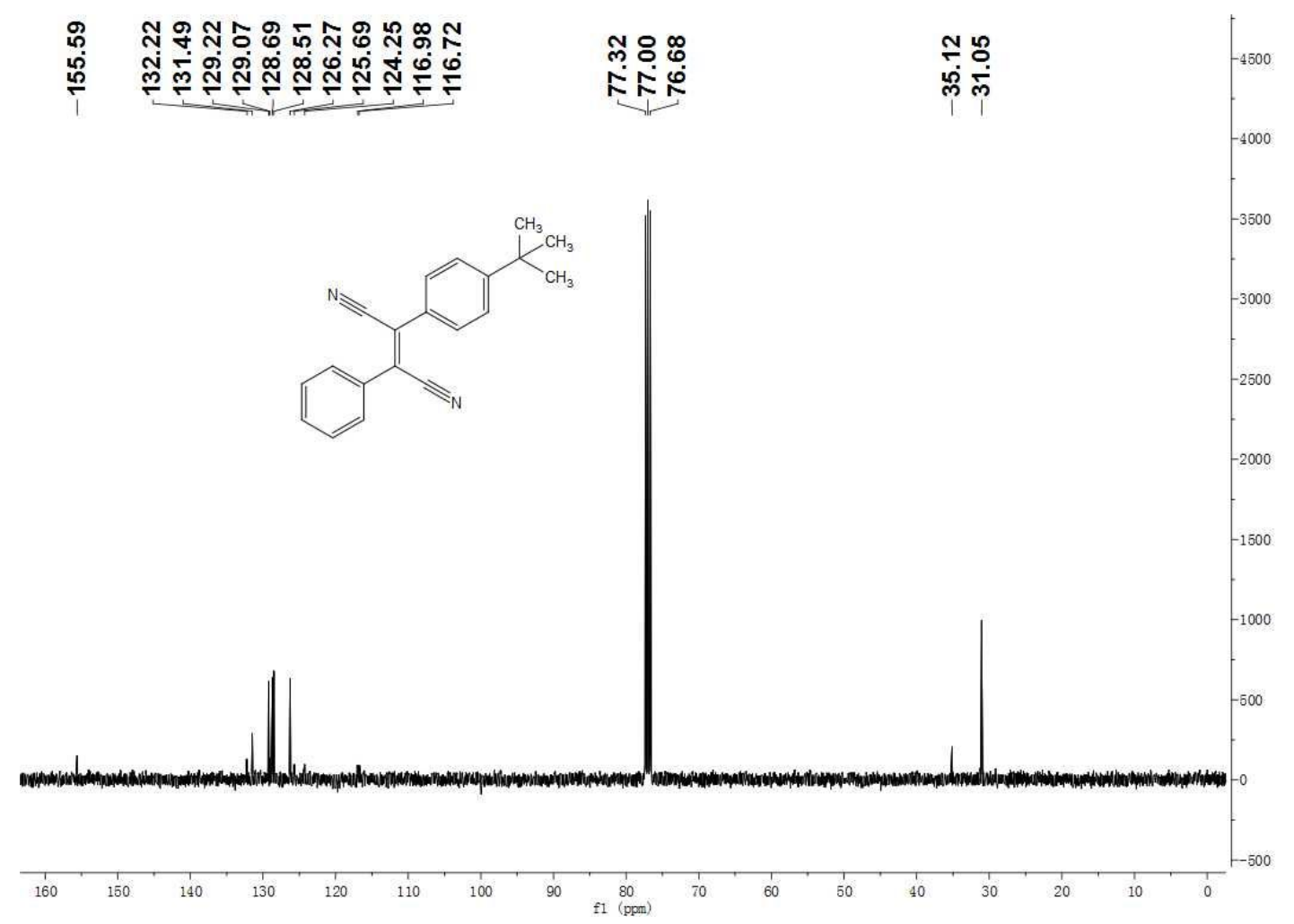

Figure S48. ${ }^{13} \mathrm{C}\left\{{ }^{1} \mathrm{H}\right\}$ NMR spectrum of $4 \mathrm{c}\left(100 \mathrm{MHz}, \mathrm{CDCl}_{3}\right)$ 


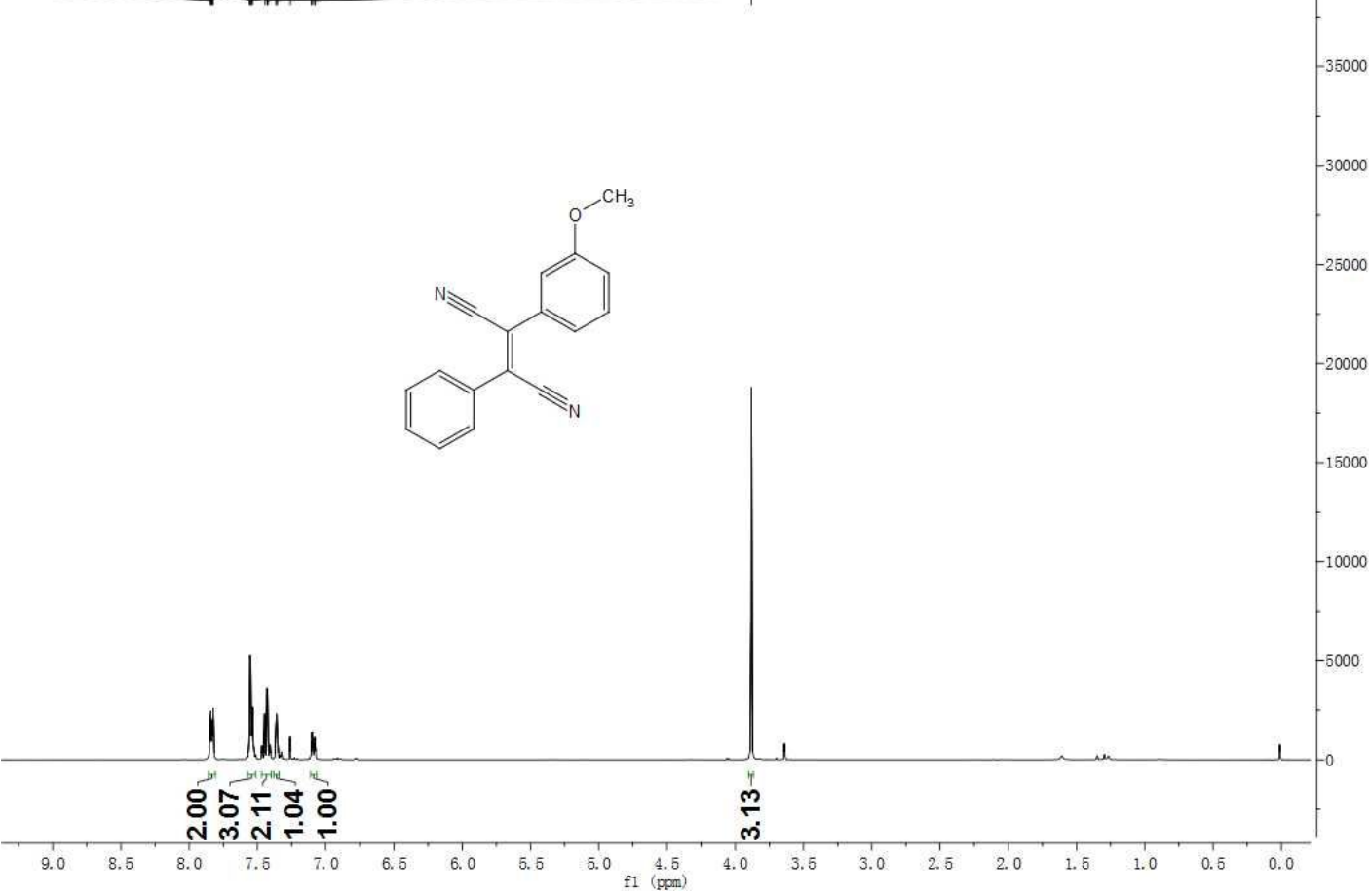

Figure S49. ${ }^{1} \mathrm{H}$ NMR spectrum of $4 \mathrm{~d}\left(400 \mathrm{MHz}, \mathrm{CDCl}_{3}\right)$

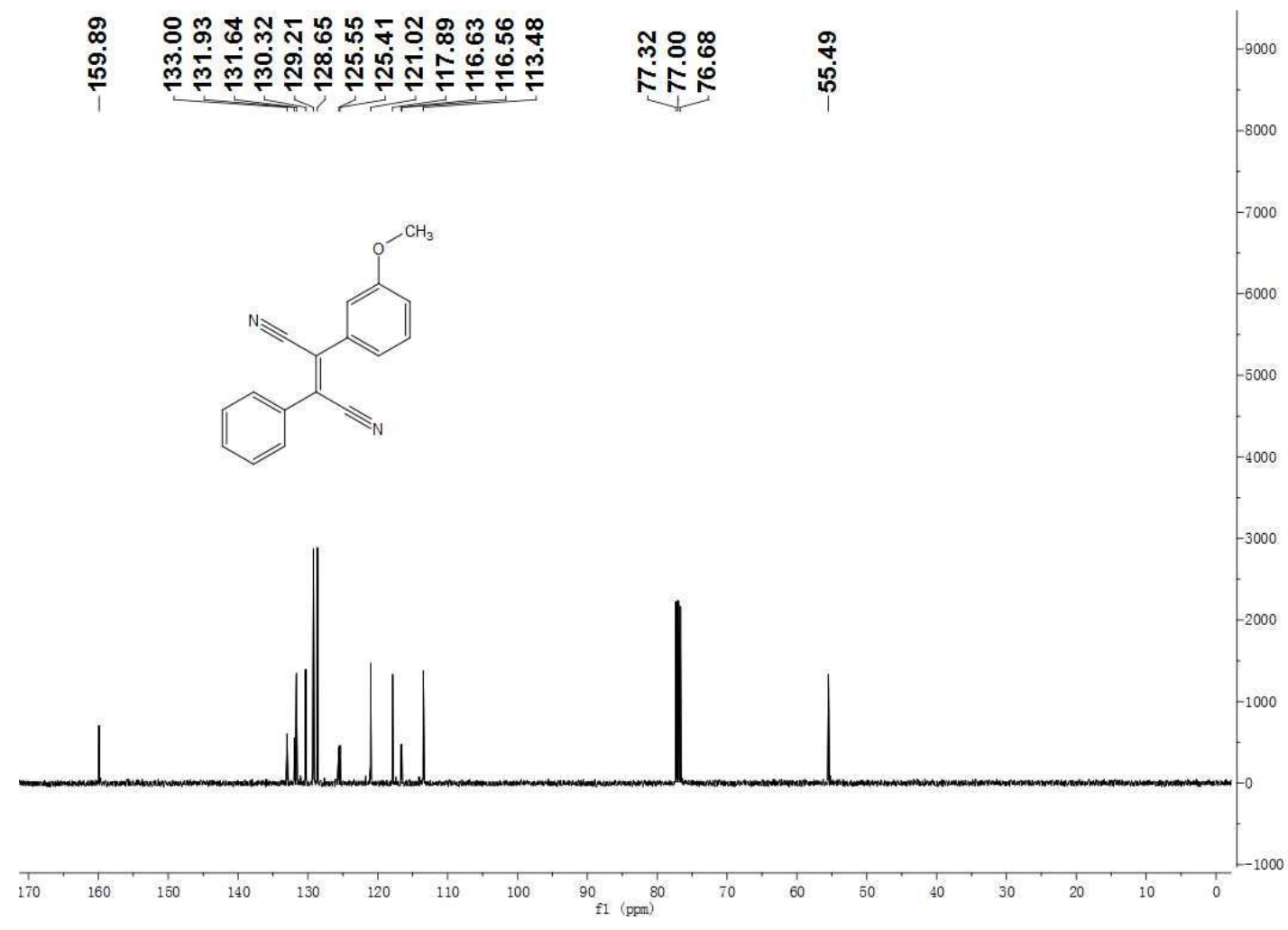

Figure S50. ${ }^{13} \mathrm{C}\left\{{ }^{1} \mathrm{H}\right\}$ NMR spectrum of $4 \mathrm{~d}\left(100 \mathrm{MHz}, \mathrm{CDCl}_{3}\right)$ 


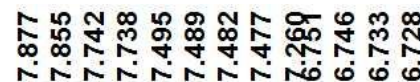

กิ

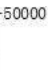

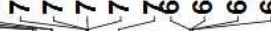
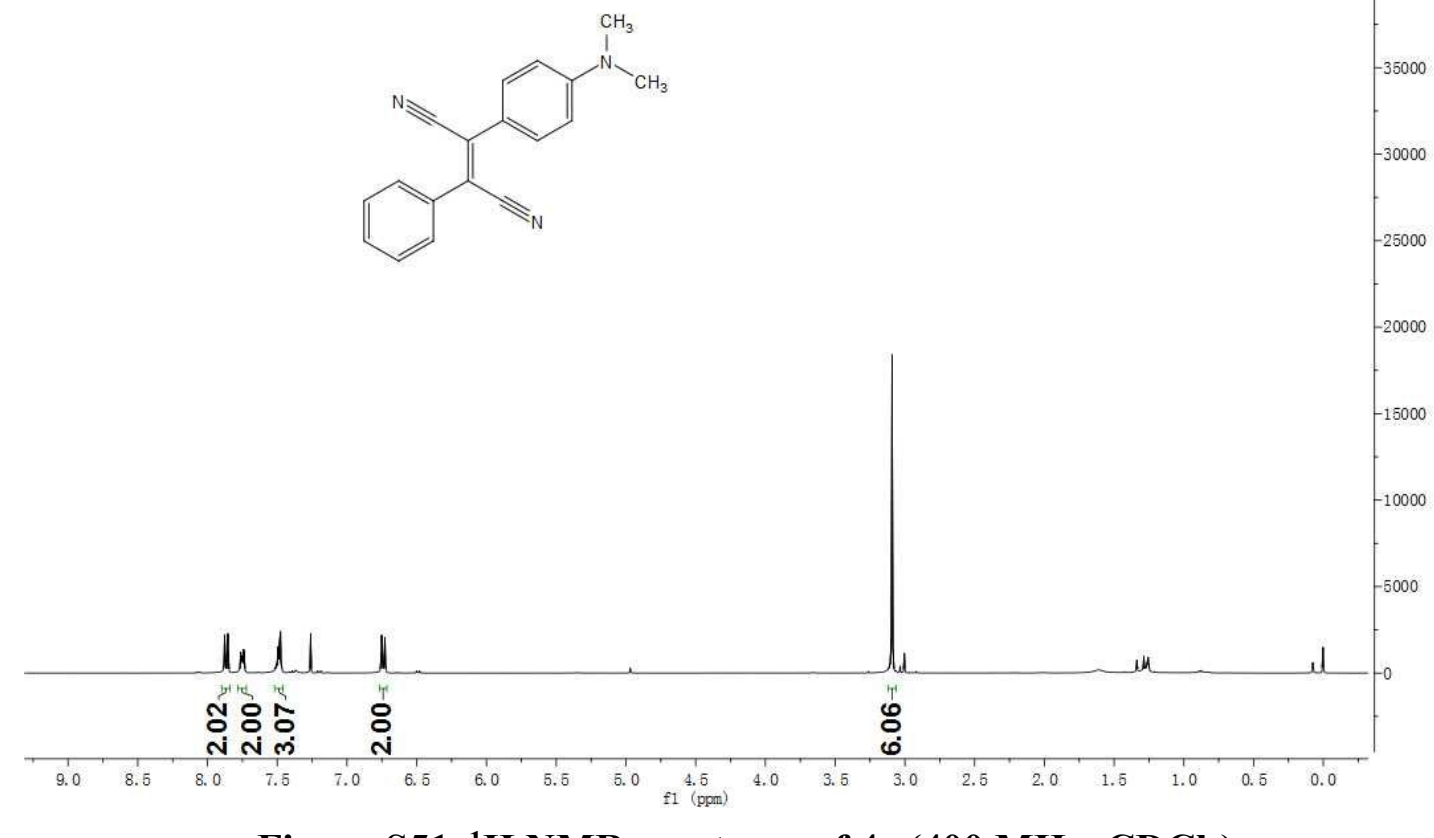

Figure S51. ${ }^{1} \mathrm{H}$ NMR spectrum of $4 \mathrm{e}\left(400 \mathrm{MHz}, \mathrm{CDCl}_{3}\right)$

โ

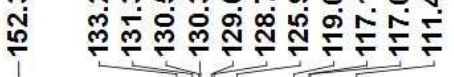

กัలి

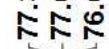

চ̊̀

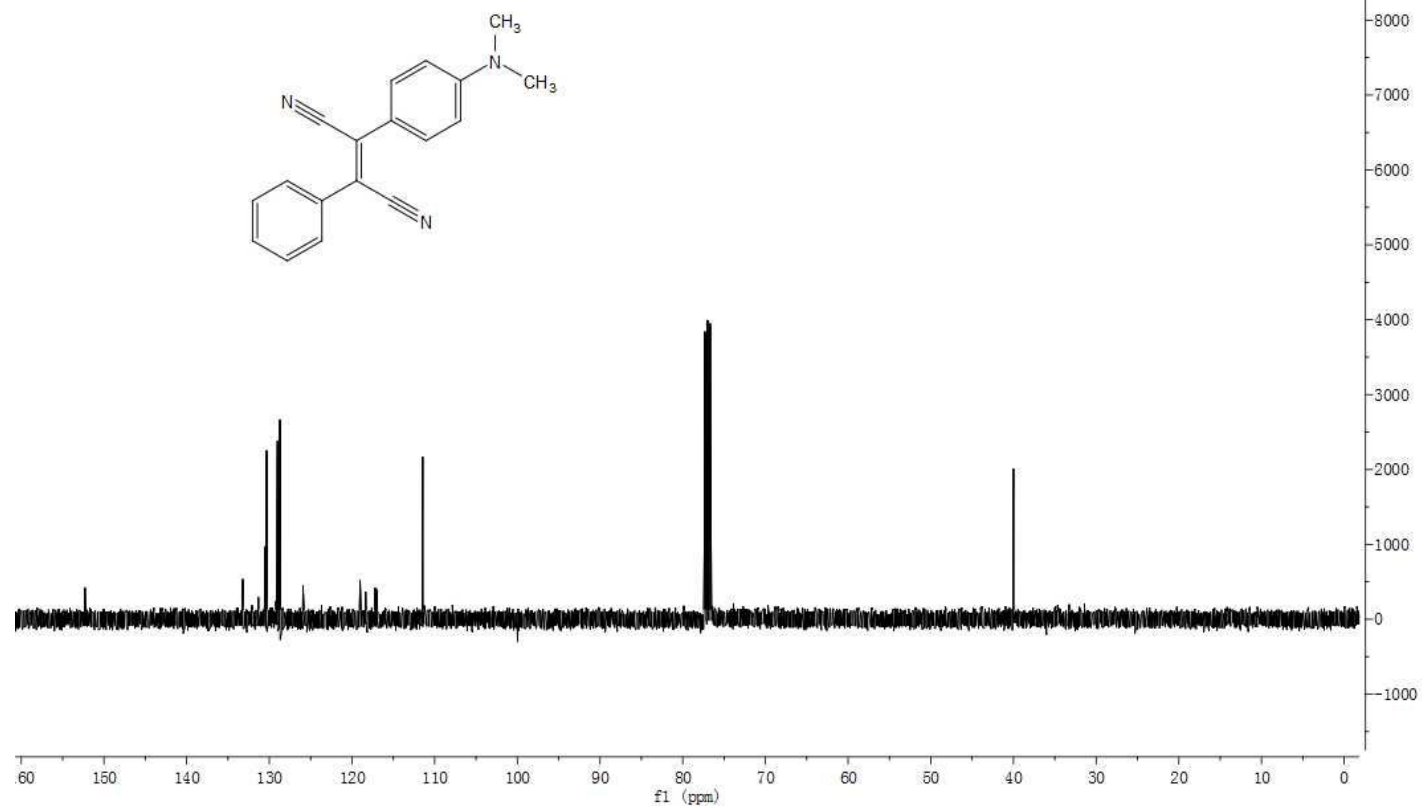

Figure S52. ${ }^{13} \mathrm{C}\left\{{ }^{1} \mathrm{H}\right\}$ NMR spectrum of $4 \mathrm{e}\left(100 \mathrm{MHz}, \mathrm{CDCl}_{3}\right)$ 


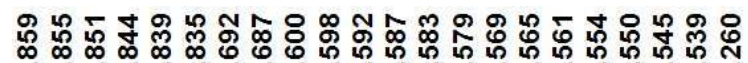

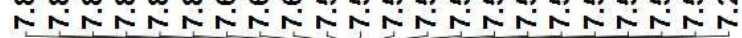

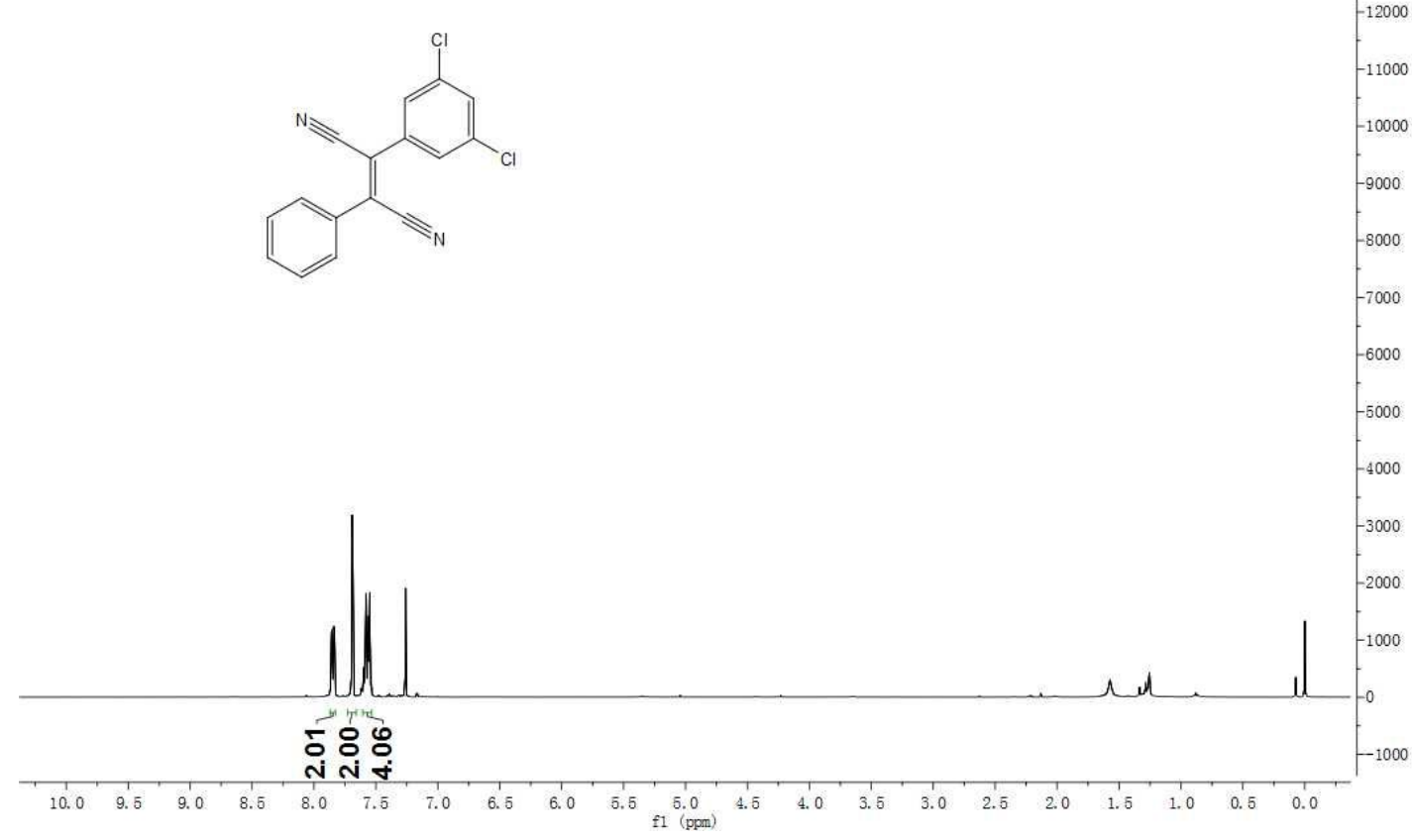

Figure S53. ${ }^{1} \mathrm{H}$ NMR spectrum of $4 \mathrm{f}\left(400 \mathrm{MHz}, \mathrm{CDCl}_{3}\right)$

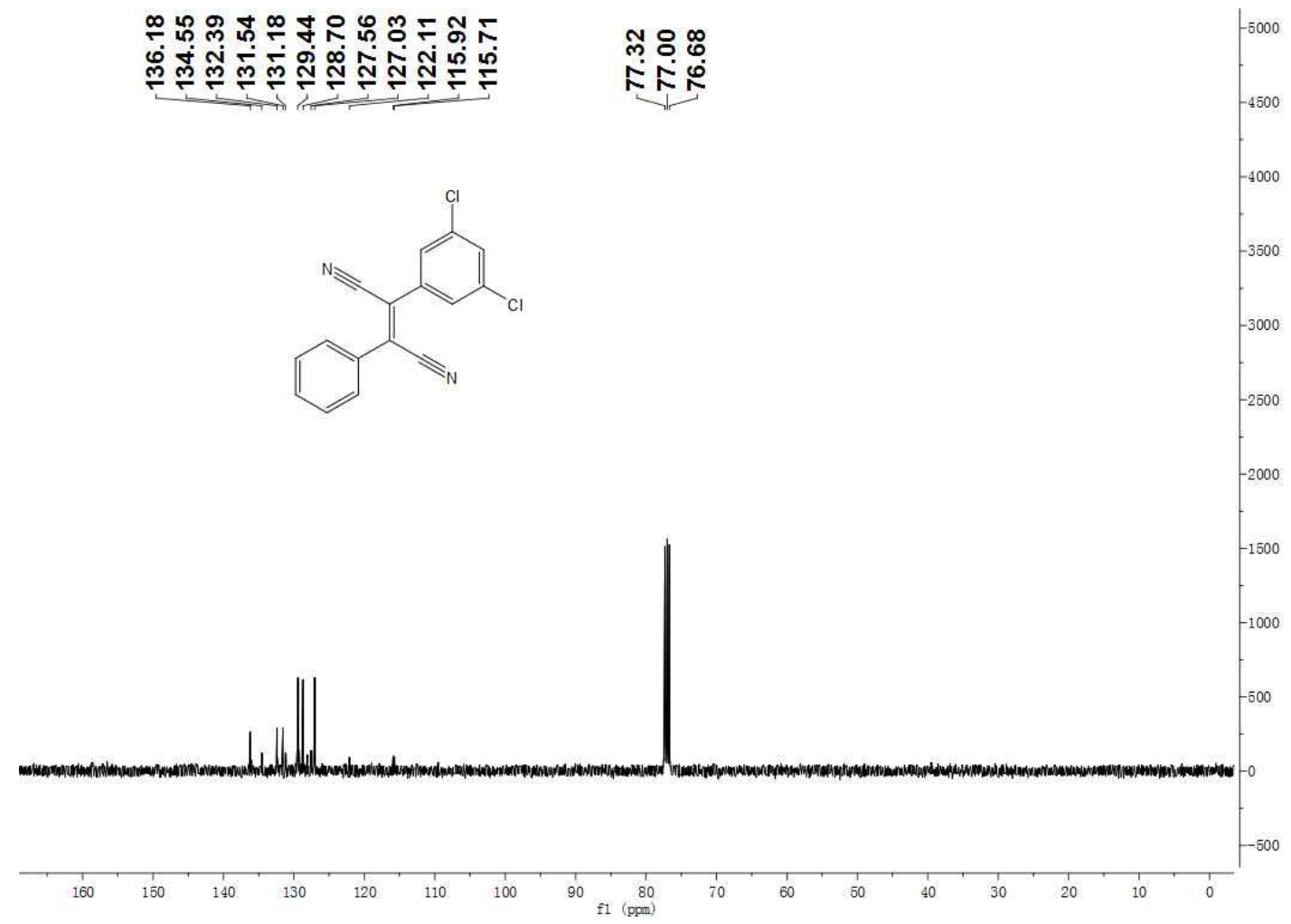

Figure S54. ${ }^{13} \mathrm{C}\left\{{ }^{1} \mathrm{H}\right\}$ NMR spectrum of $4 \mathrm{f}\left(100 \mathrm{MHz}, \mathrm{CDCl}_{3}\right)$ 


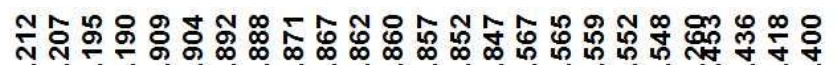

禹市
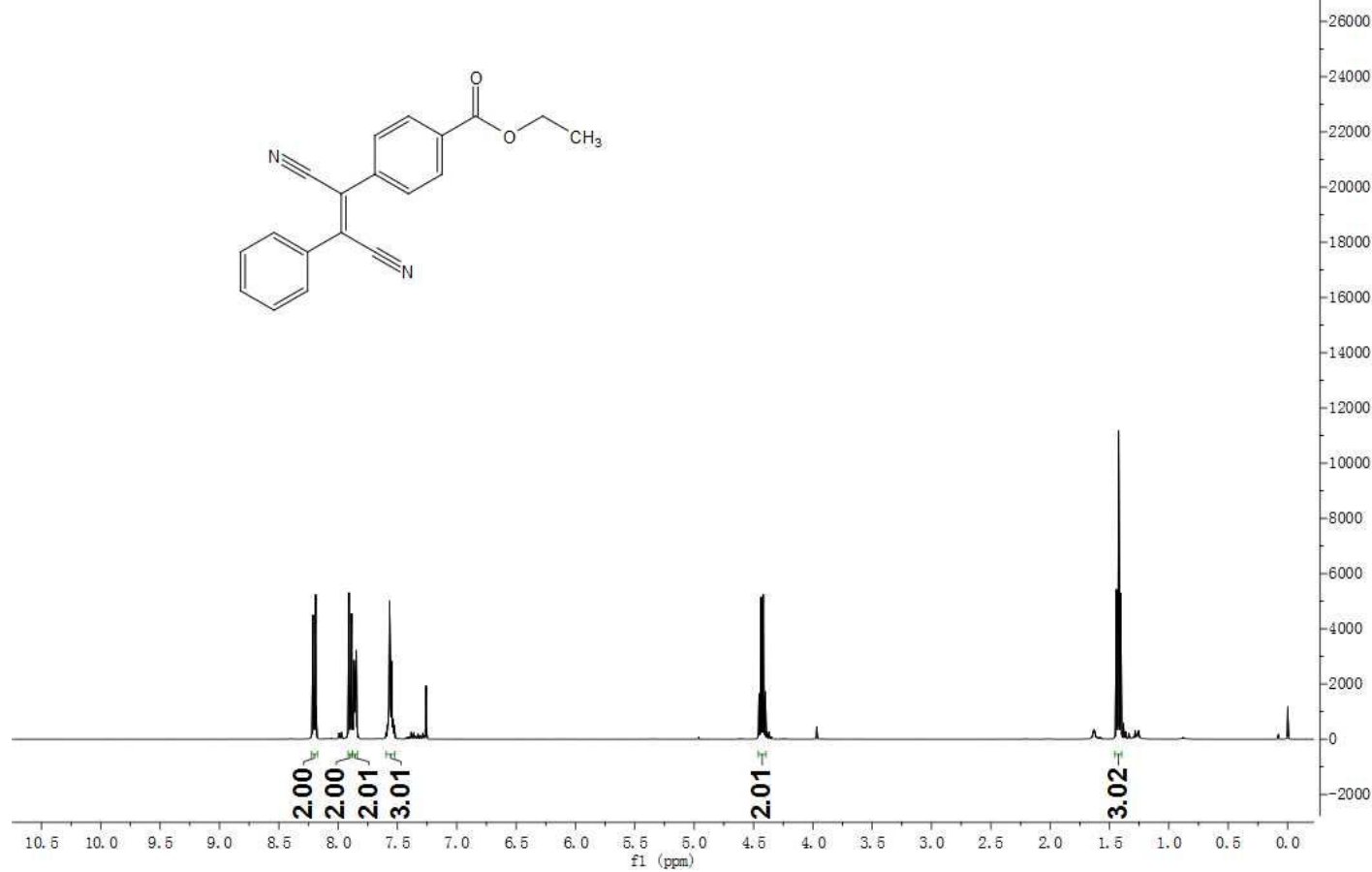

Figure S55. ${ }^{1} \mathrm{H}$ NMR spectrum of $4 \mathrm{~g}\left(400 \mathrm{MHz}, \mathrm{CDCl}_{3}\right)$

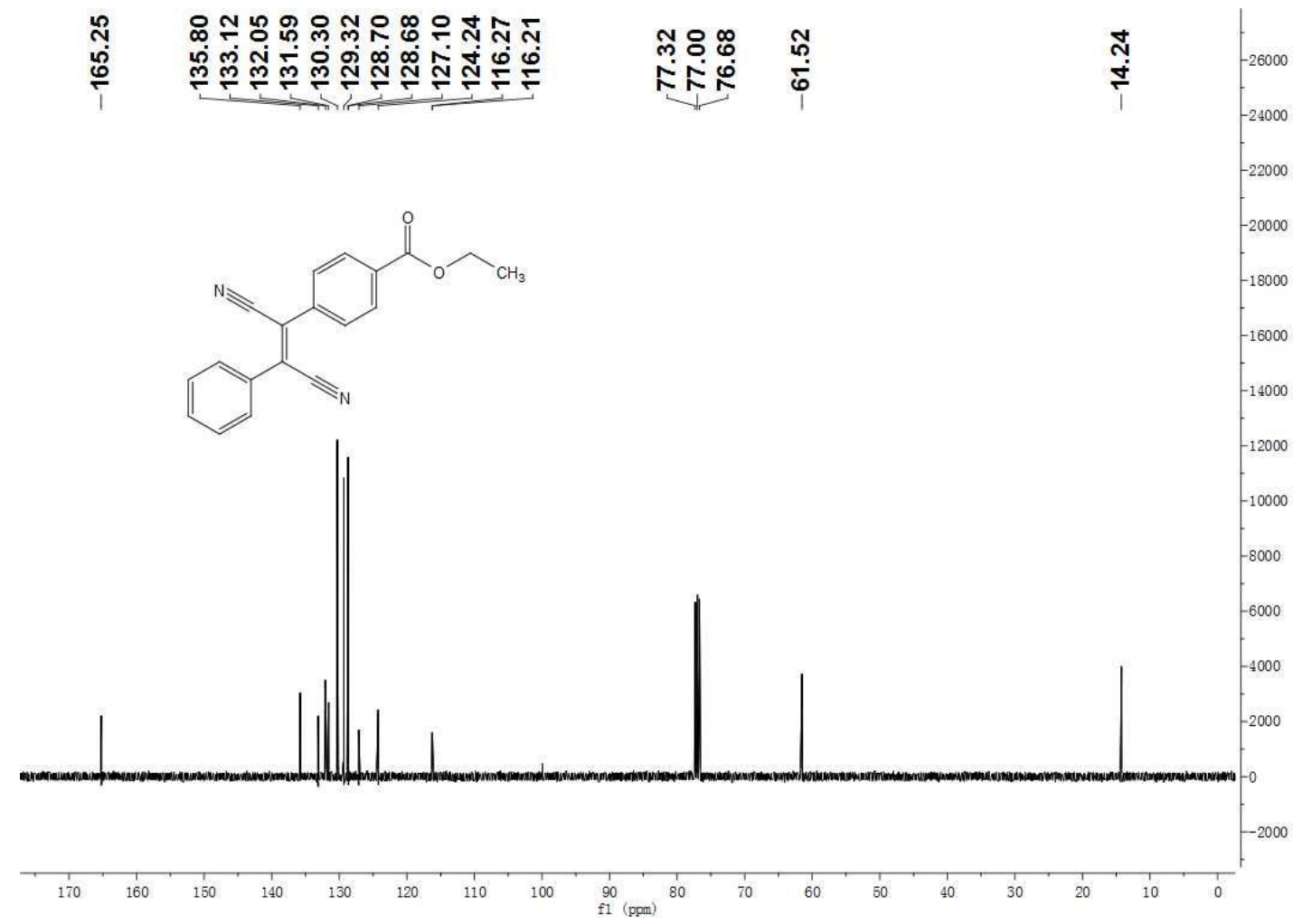

Figure S56. ${ }^{13} \mathrm{C}\left\{{ }^{1} \mathrm{H}\right\}$ NMR spectrum of $4 \mathrm{~g}\left(100 \mathrm{MHz}, \mathrm{CDCl}_{3}\right)$ 


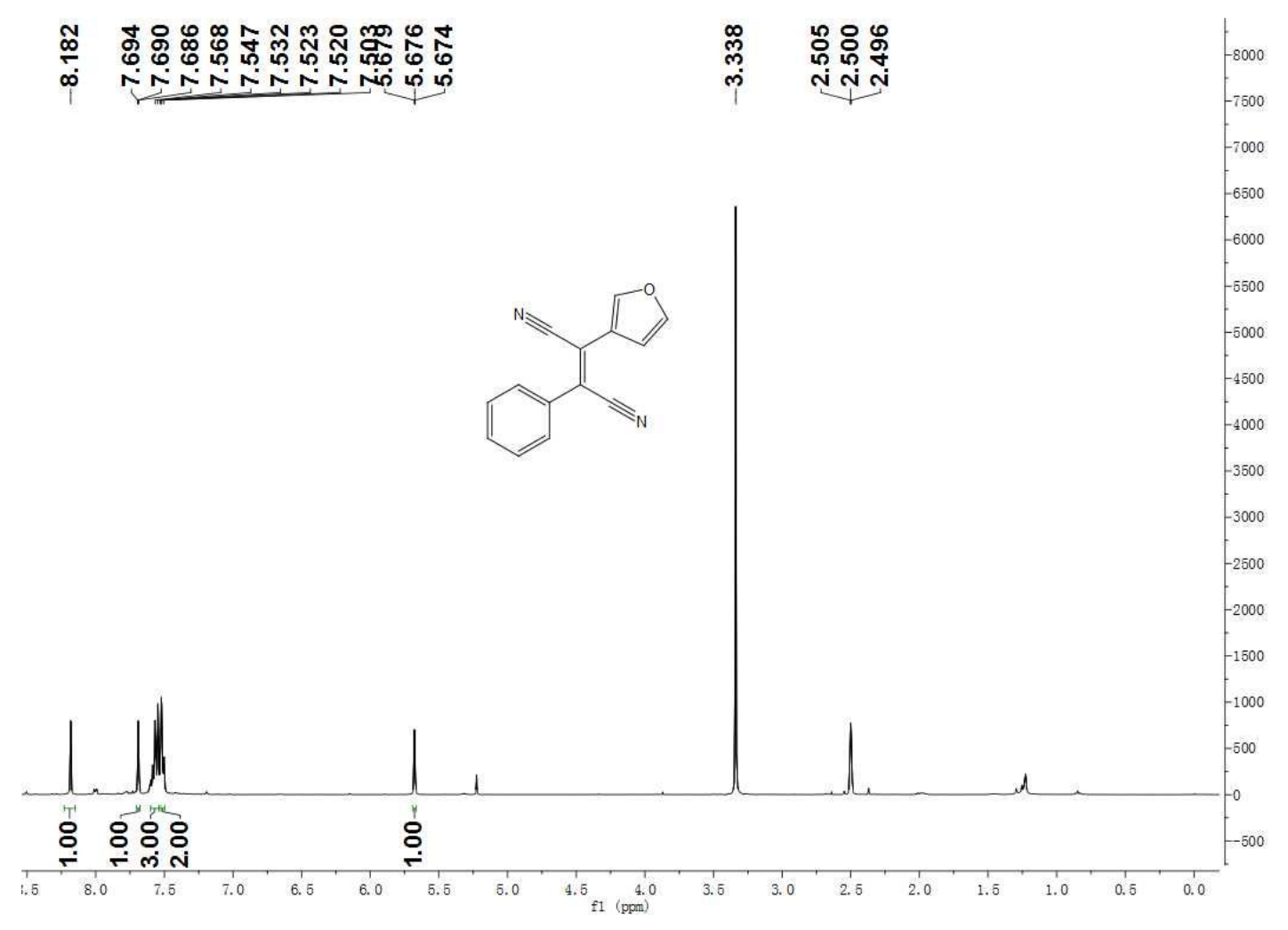

Figure S57. ${ }^{1} \mathrm{H}$ NMR spectrum of $4 \mathrm{~h}(400 \mathrm{MHz}$, DMSO-d6)

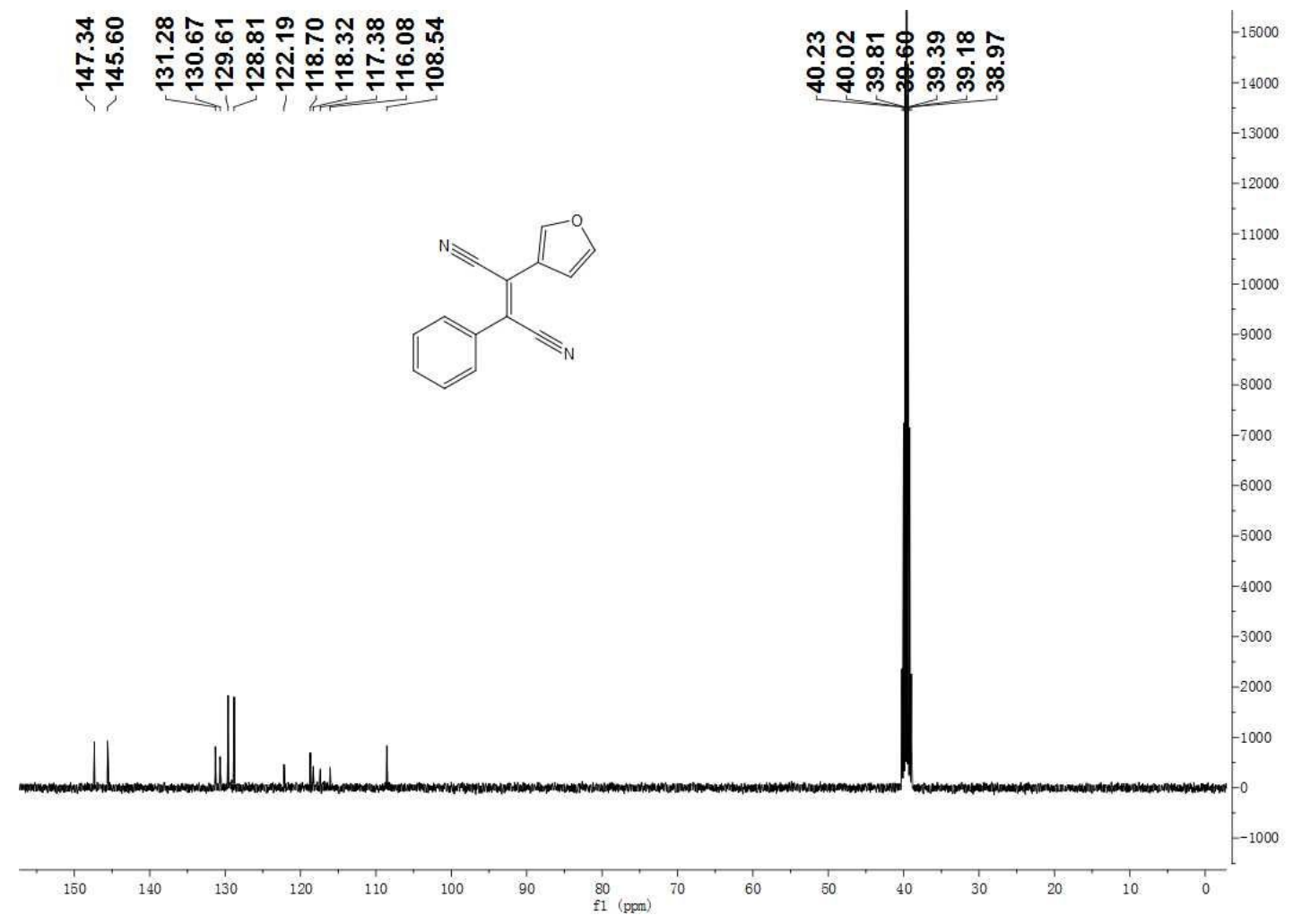

Figure S58. ${ }^{13} \mathrm{C}\left\{{ }^{1} \mathrm{H}\right\}$ NMR spectrum of $4 \mathrm{~h}(100 \mathrm{MHz}, \mathrm{DMSO}-d 6)$ 


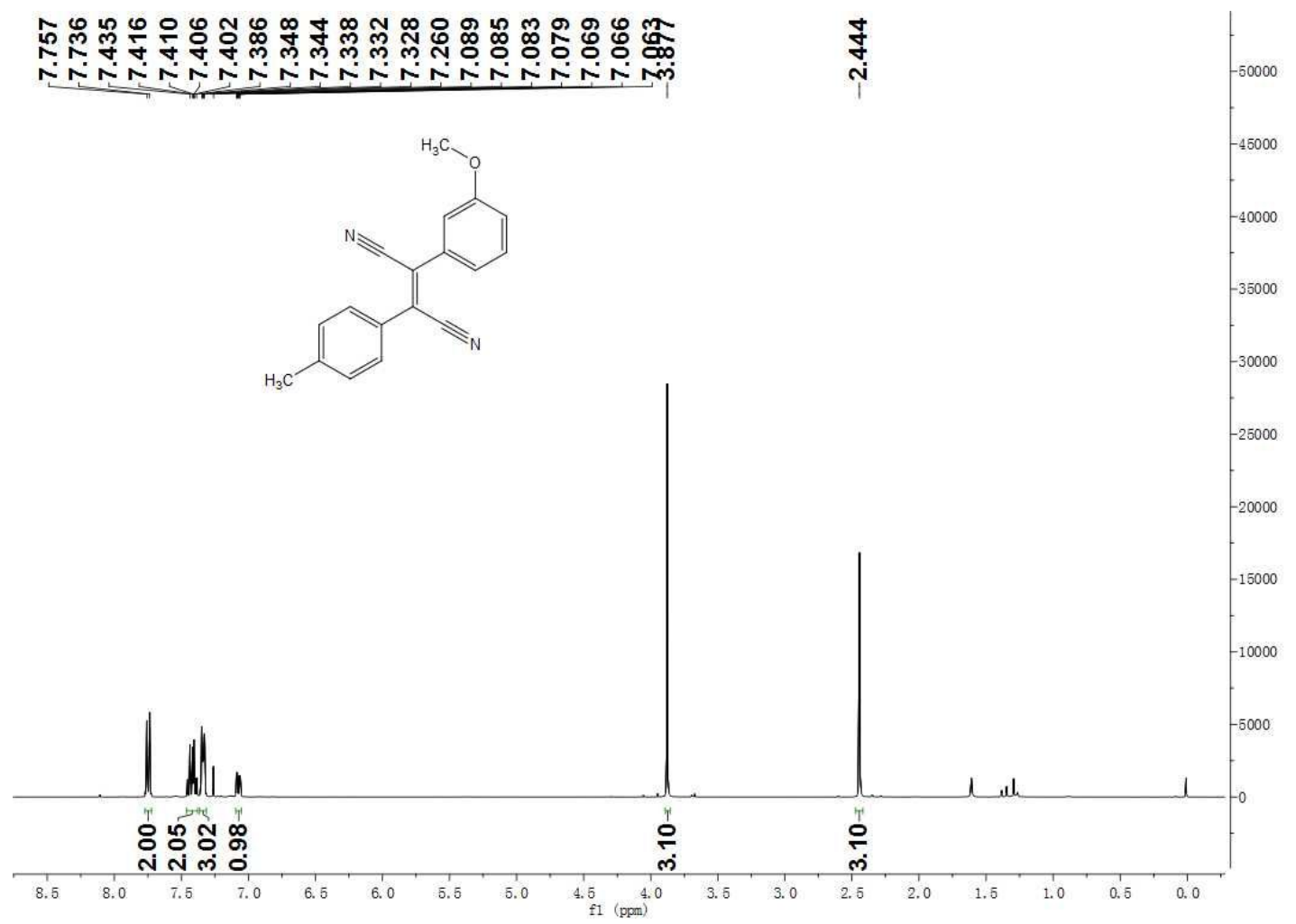

Figure S59. ${ }^{1} \mathrm{H}$ NMR spectrum of $4 \mathrm{i}\left(400 \mathrm{MHz}, \mathrm{CDCl}_{3}\right)$

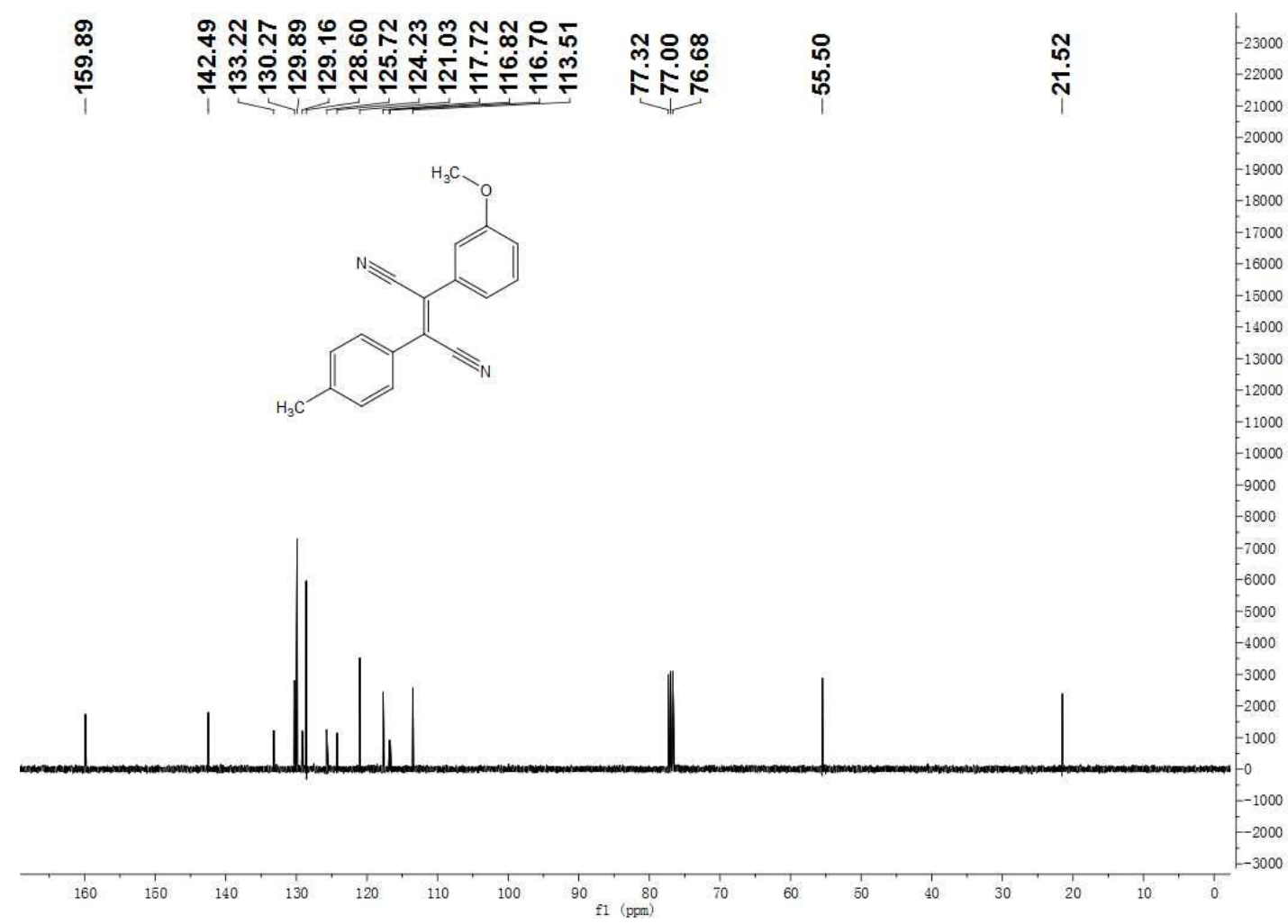

Figure S60. ${ }^{13} \mathrm{C}\left\{{ }^{1} \mathrm{H}\right\}$ NMR spectrum of $4 \mathrm{i}\left(100 \mathrm{MHz}, \mathrm{CDCl}_{3}\right)$ 


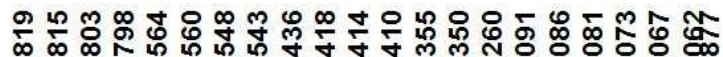

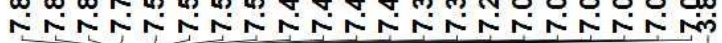
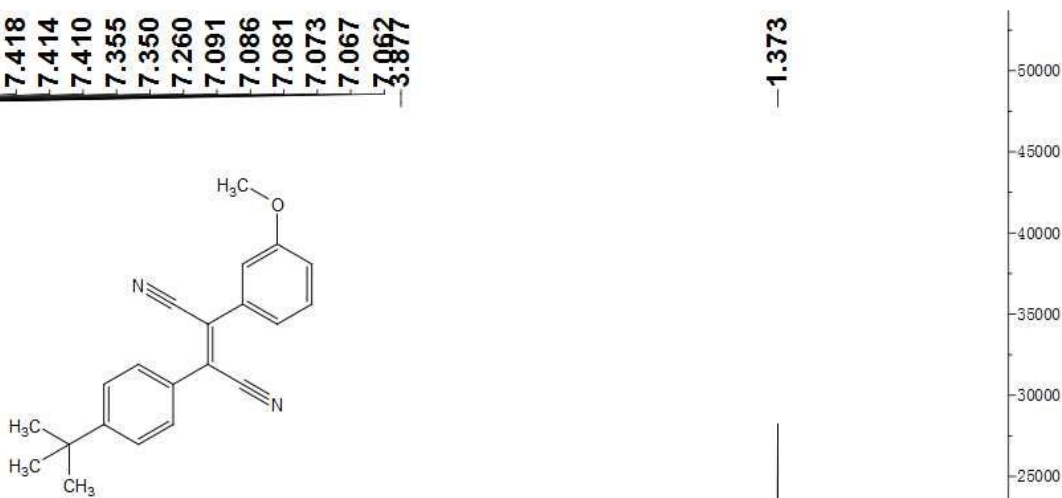

1 Hu.

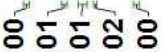

n n N

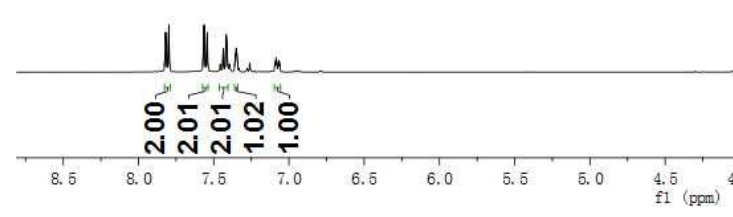

Figure S61. ${ }^{1} \mathrm{H}$ NMR spectrum of $4 \mathrm{j}\left(400 \mathrm{MHz}, \mathrm{CDCl}_{3}\right)$

๘

官 以

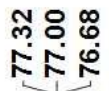

号

\%ั̊ :

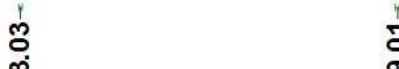

$\delta_{0}^{4}$
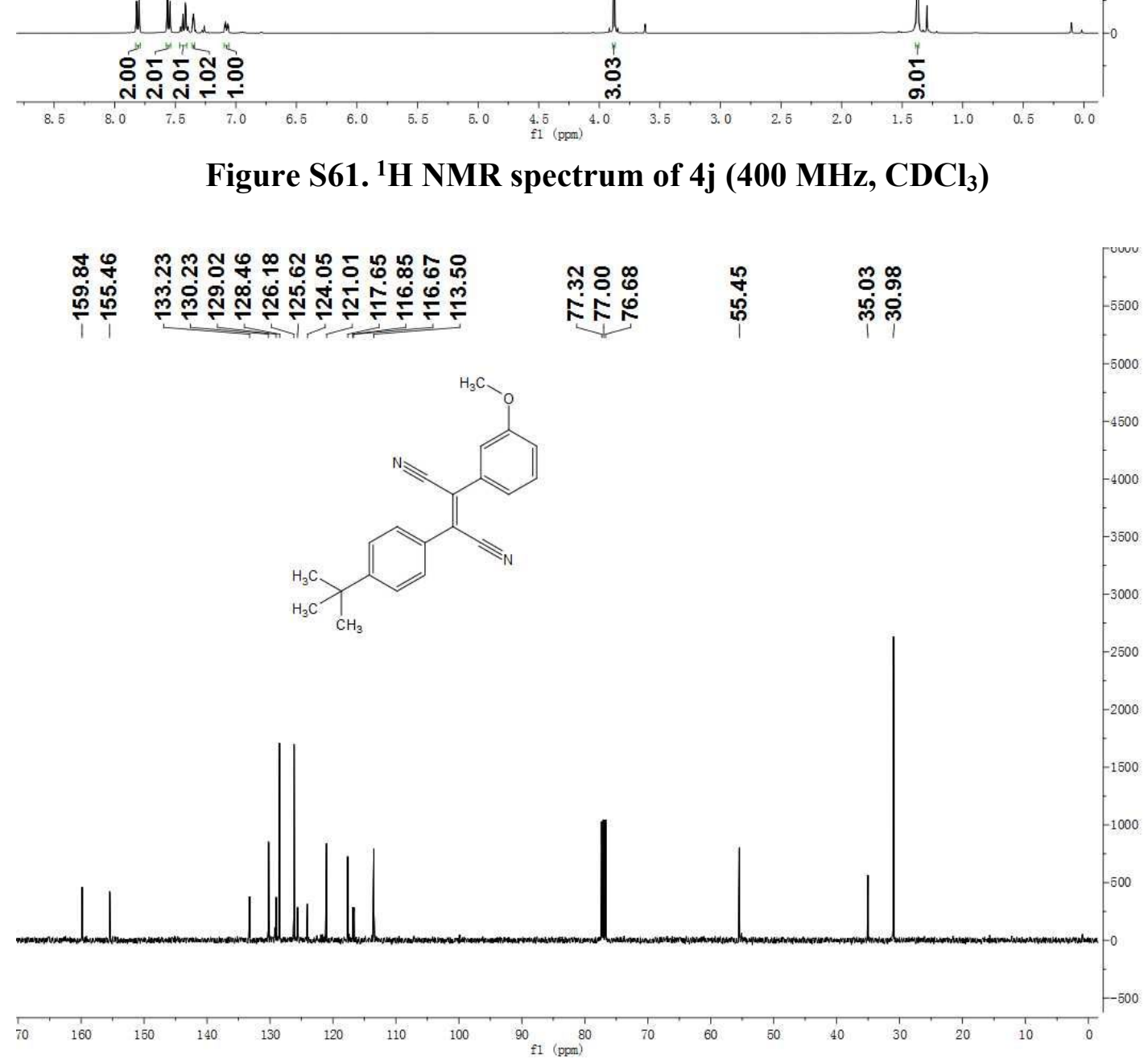

КNए म

Figure S62. ${ }^{13} \mathrm{C}\left\{{ }^{1} \mathrm{H}\right\}$ NMR spectrum of $4 \mathrm{j}\left(100 \mathrm{MHz}, \mathrm{CDCl}_{3}\right)$ 


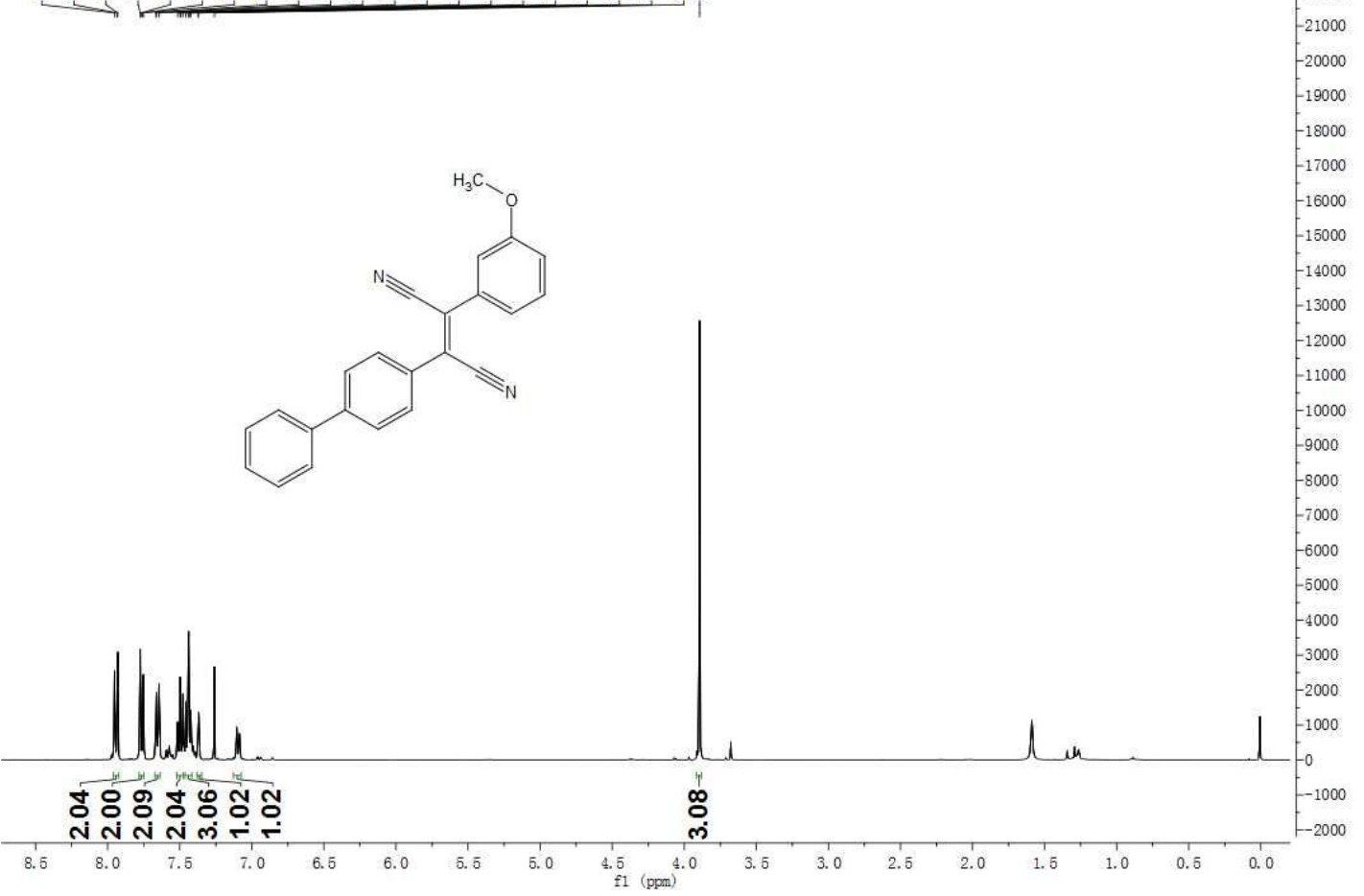

Figure S63. ${ }^{1} \mathrm{H}$ NMR spectrum of $4 \mathrm{k}\left(400 \mathrm{MHz}, \mathrm{CDCl}_{3}\right)$

นำㅇำ

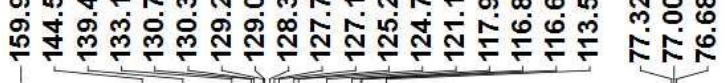

屯़

$-8000$

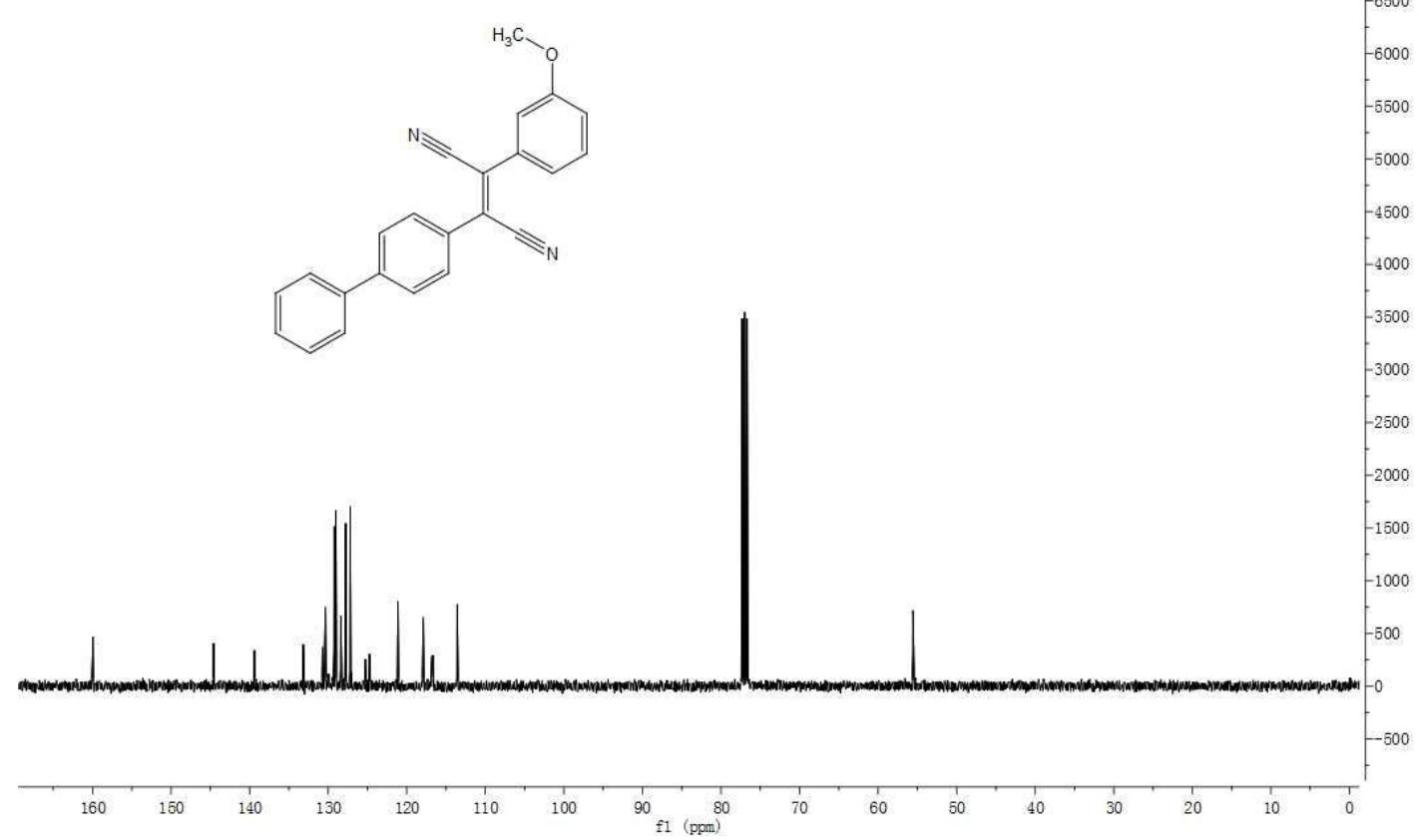

Figure S64. ${ }^{13} \mathrm{C}\left\{{ }^{1} \mathrm{H}\right\}$ NMR spectrum of $4 \mathrm{k}\left(100 \mathrm{MHz}, \mathrm{CDCl}_{3}\right)$ 


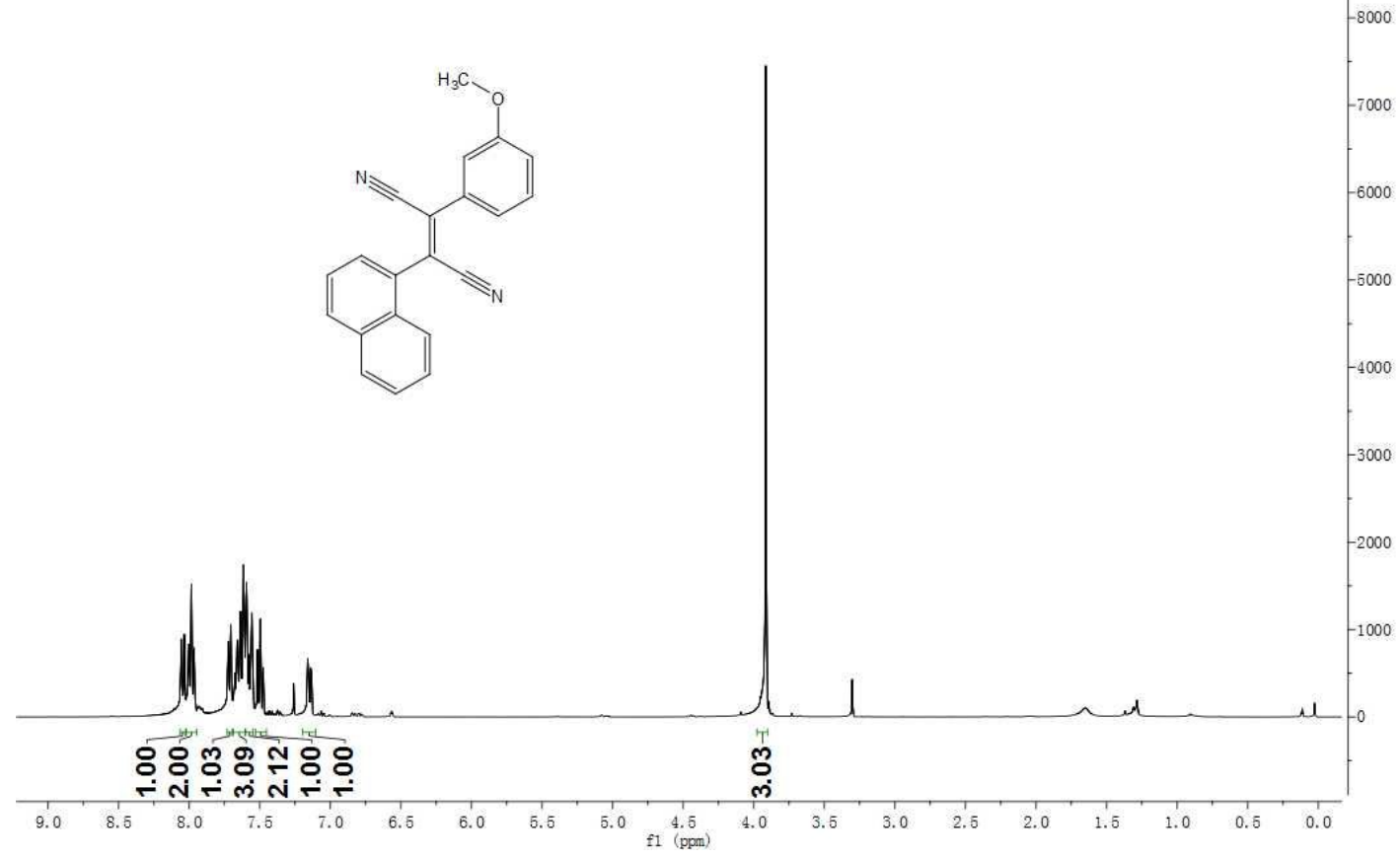

Figure S65. ${ }^{1} \mathrm{H}$ NMR spectrum of $41\left(400 \mathrm{MHz}, \mathrm{CDCl}_{3}\right)$

8.

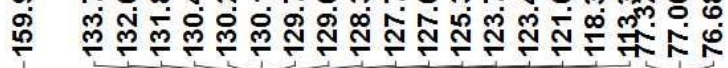

फ़

官

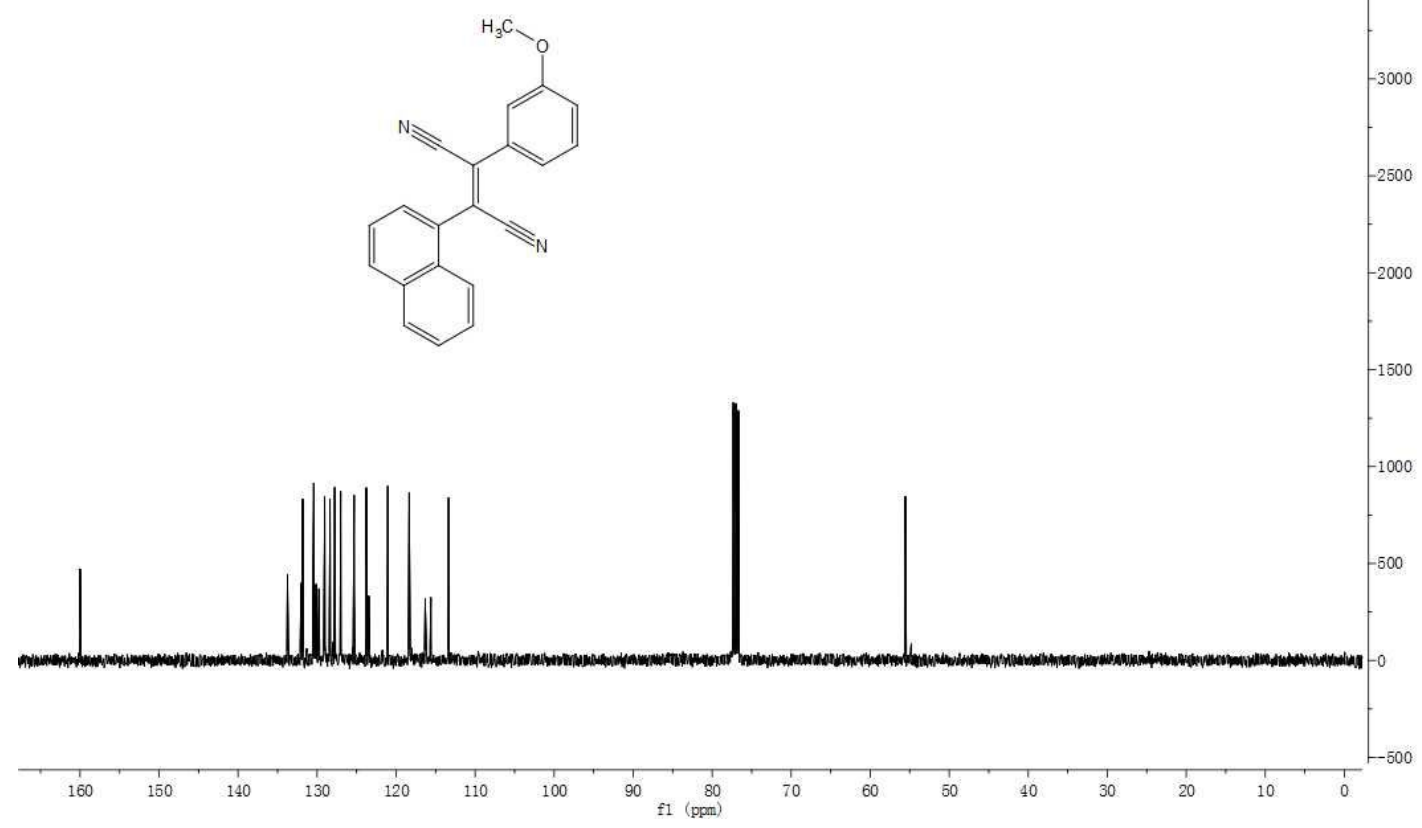

Figure S66. ${ }^{13} \mathrm{C}\left\{{ }^{1} \mathrm{H}\right\}$ NMR spectrum of $4 \mathrm{l}\left(100 \mathrm{MHz}, \mathrm{CDCl}_{3}\right)$ 
นึ

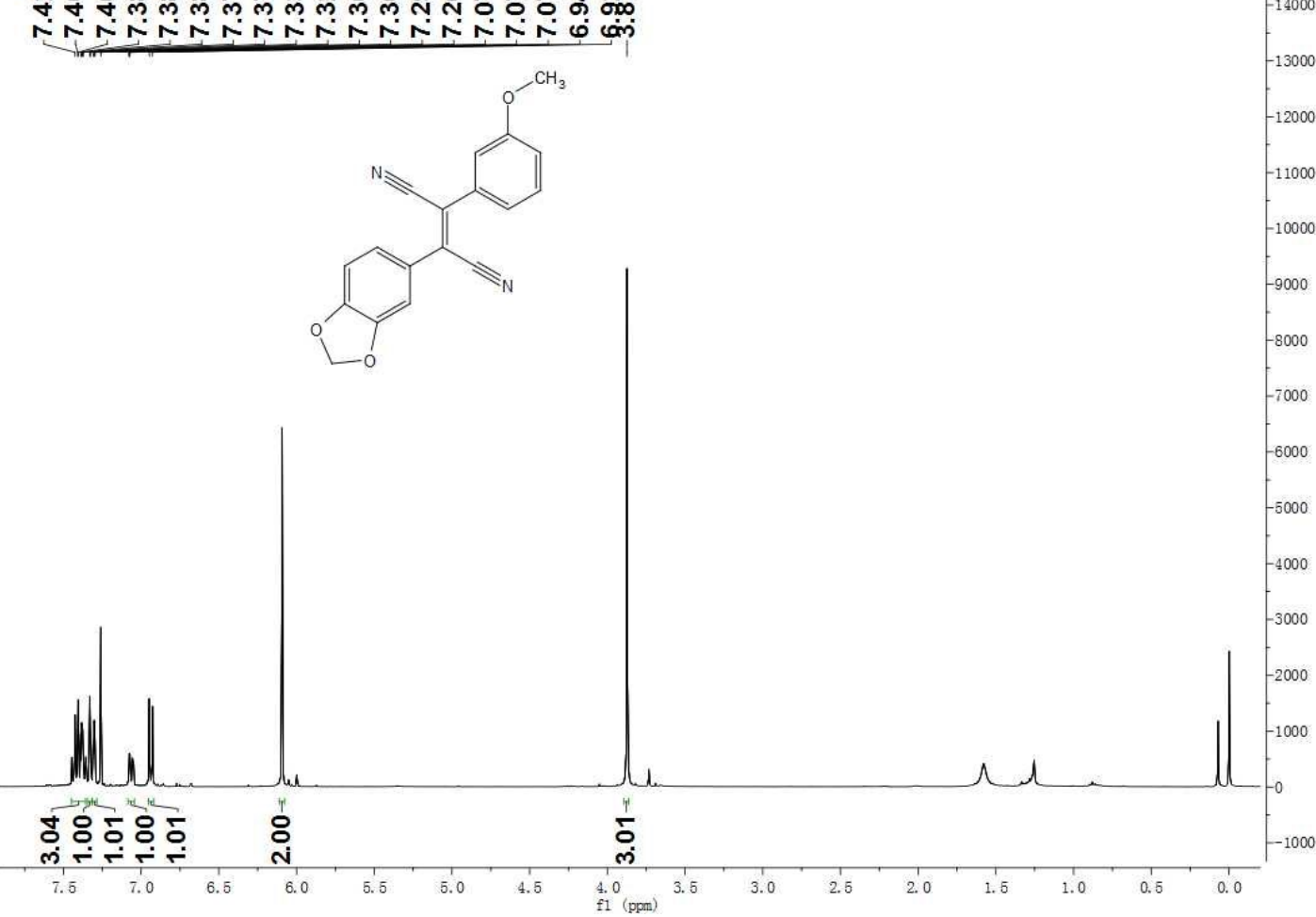

Figure S67. ${ }^{1} \mathrm{H}$ NMR spectrum of $4 \mathrm{~m}\left(400 \mathrm{MHz} \mathrm{CDCl}_{3}\right)$

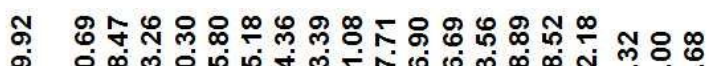

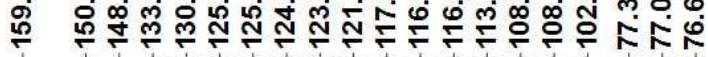

ณึ

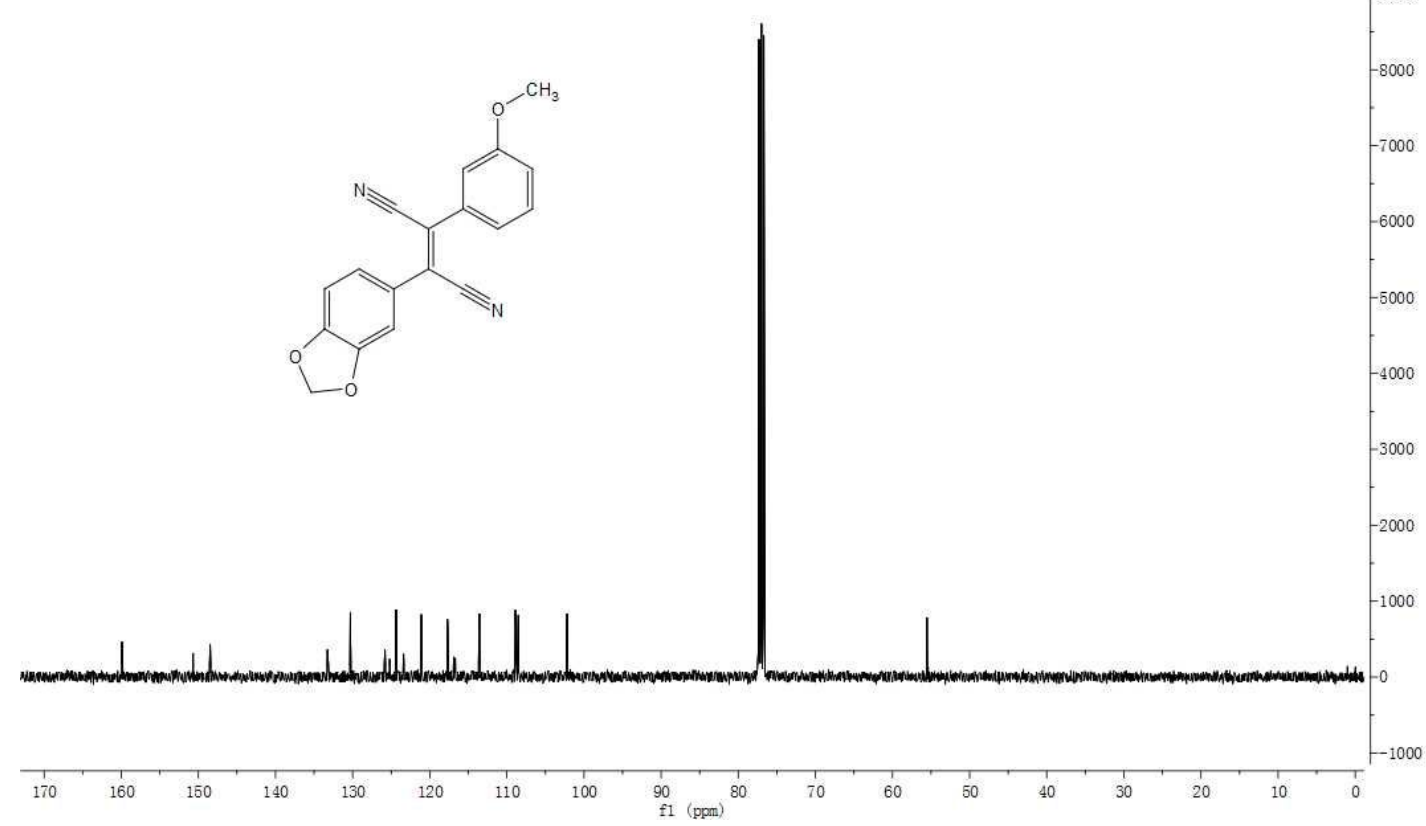

Figure S68. ${ }^{13} \mathrm{C}\left\{{ }^{1} \mathrm{H}\right\}$ NMR spectrum of $4 \mathrm{~m}\left(100 \mathrm{MHz}, \mathrm{CDCl}_{3}\right)$ 


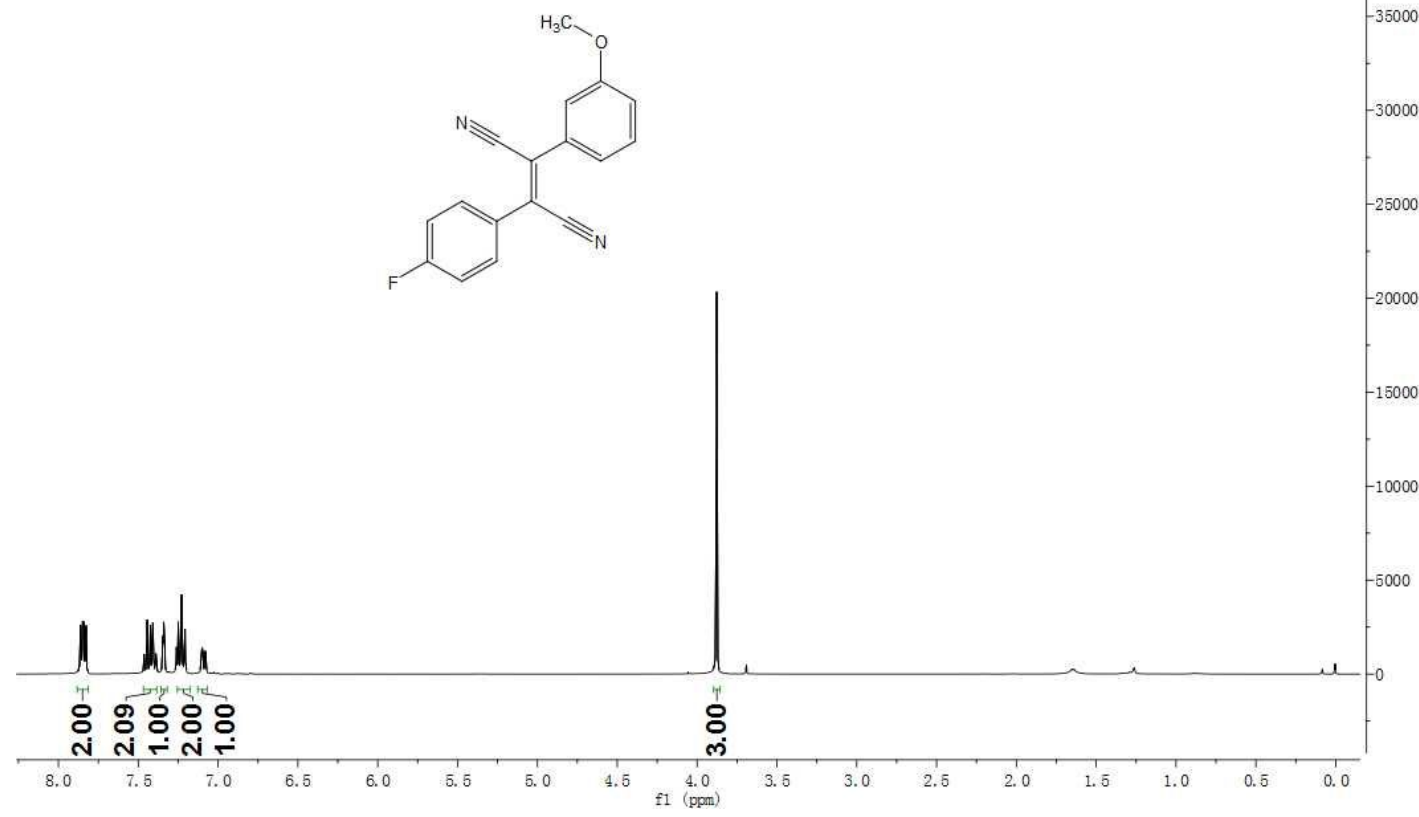

Figure S69. ${ }^{1} \mathrm{H}$ NMR spectrum of $4 \mathrm{n}\left(400 \mathrm{MHz}, \mathrm{CDCl}_{3}\right)$

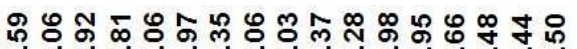

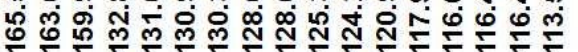

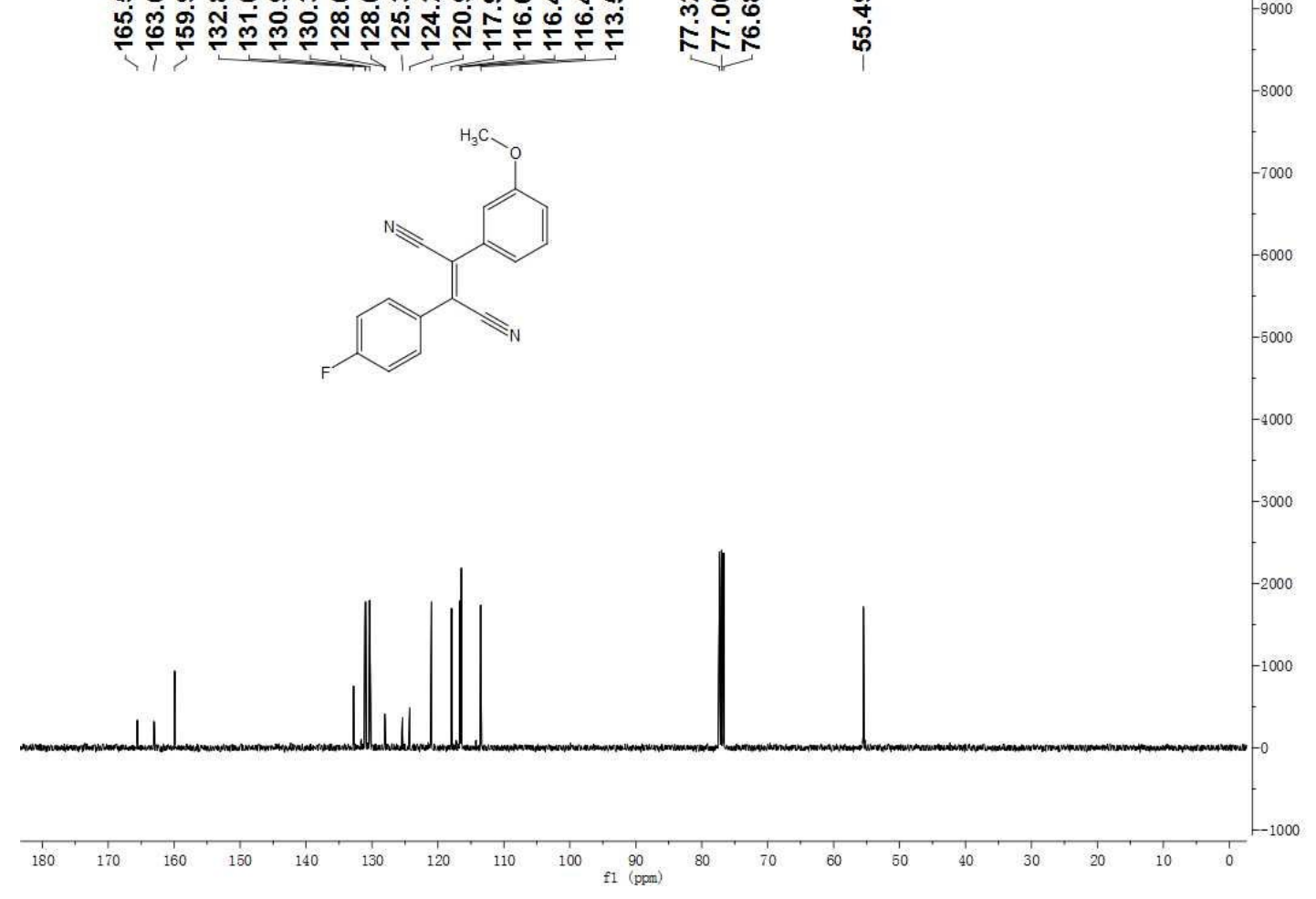

Figure $S 70 .{ }^{13} \mathrm{C}\left\{{ }^{1} \mathrm{H}\right\}$ NMR spectrum of $4 \mathrm{n}\left(100 \mathrm{MHz}, \mathrm{CDCl}_{3}\right)$ 


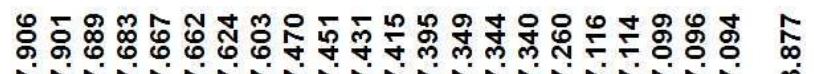

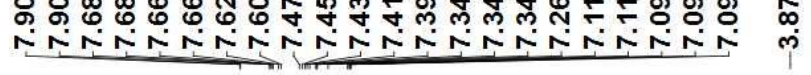

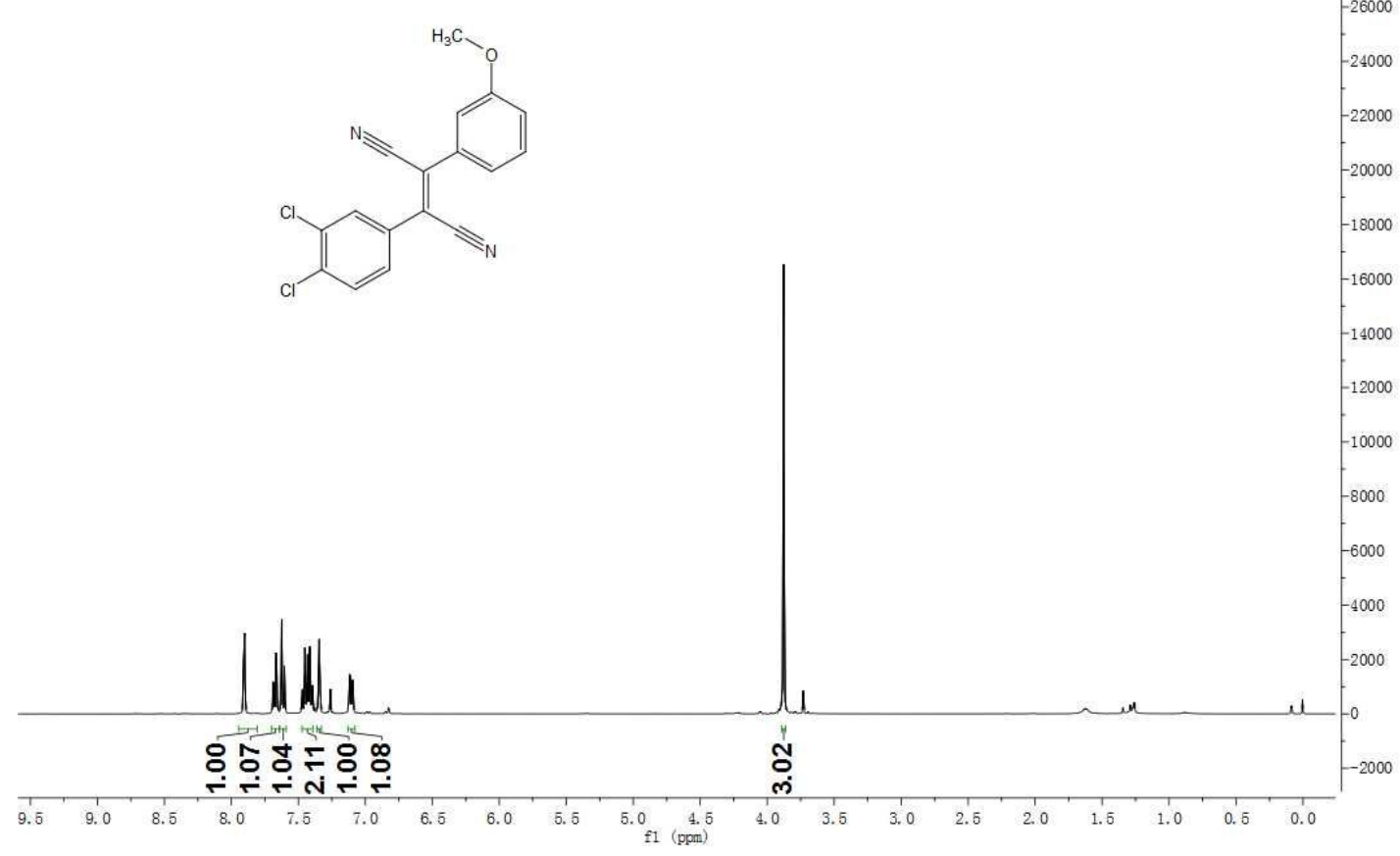

Figure S71. ${ }^{1} \mathrm{H}$ NMR spectrum of $40\left(400 \mathrm{MHz}, \mathrm{CDCl}_{3}\right)$

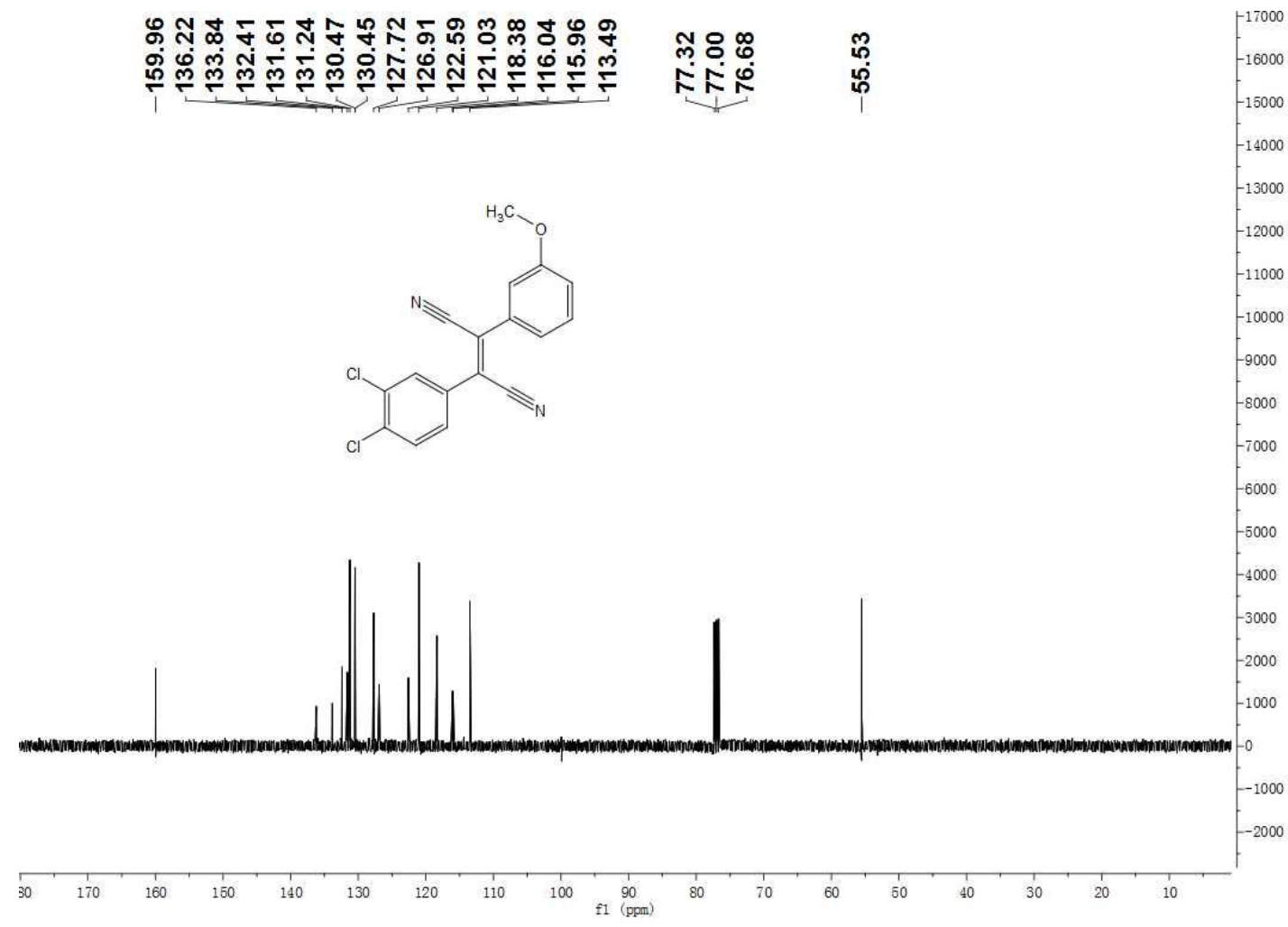

Figure S72. ${ }^{13} \mathrm{C}\left\{{ }^{1} \mathrm{H}\right\}$ NMR spectrum of $40\left(100 \mathrm{MHz}, \mathrm{CDCl}_{3}\right)$ 


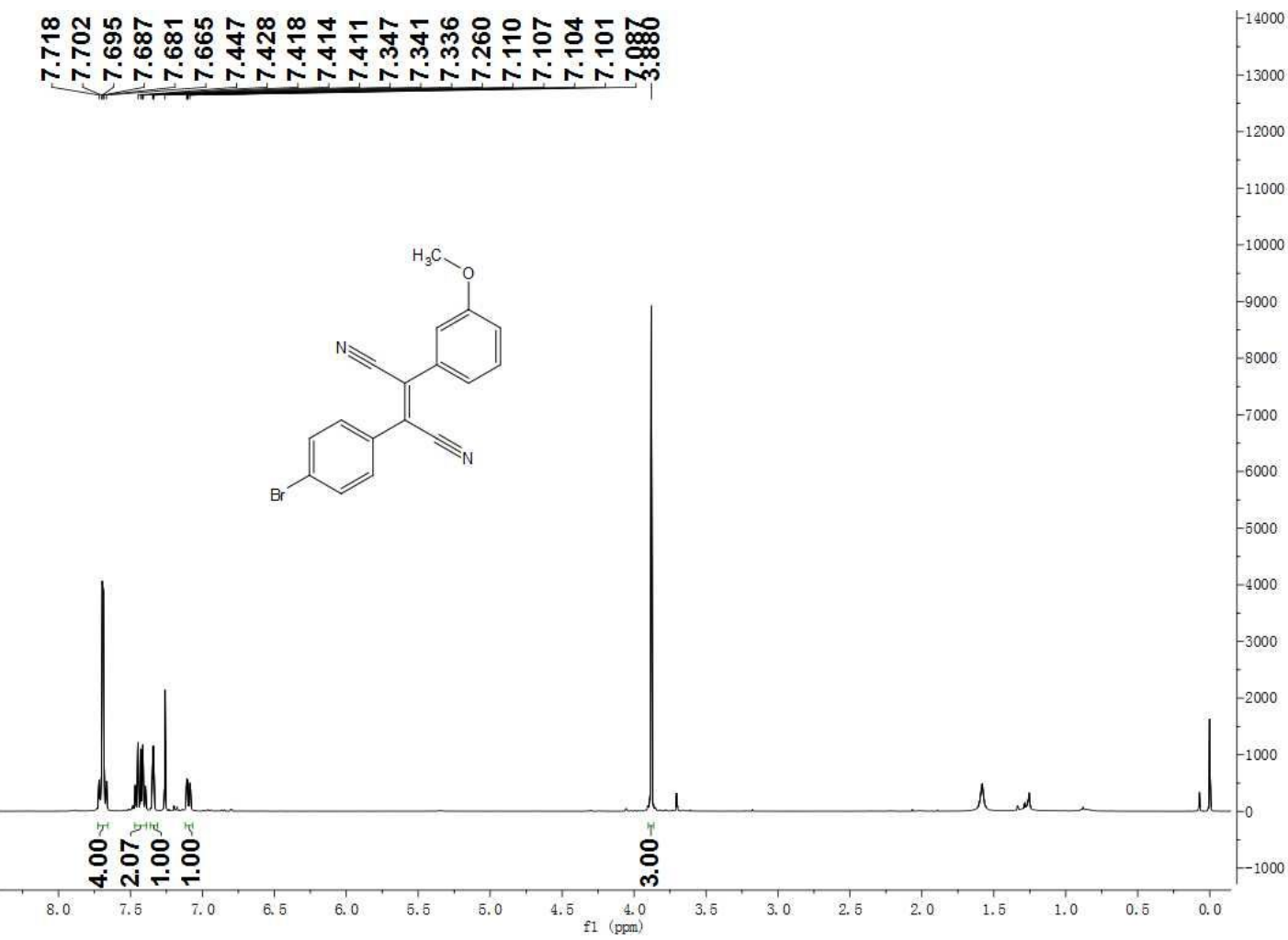

Figure S73. ${ }^{1} \mathrm{H}$ NMR spectrum of $4 \mathrm{p}\left(400 \mathrm{MHz}, \mathrm{CDCl}_{3}\right)$

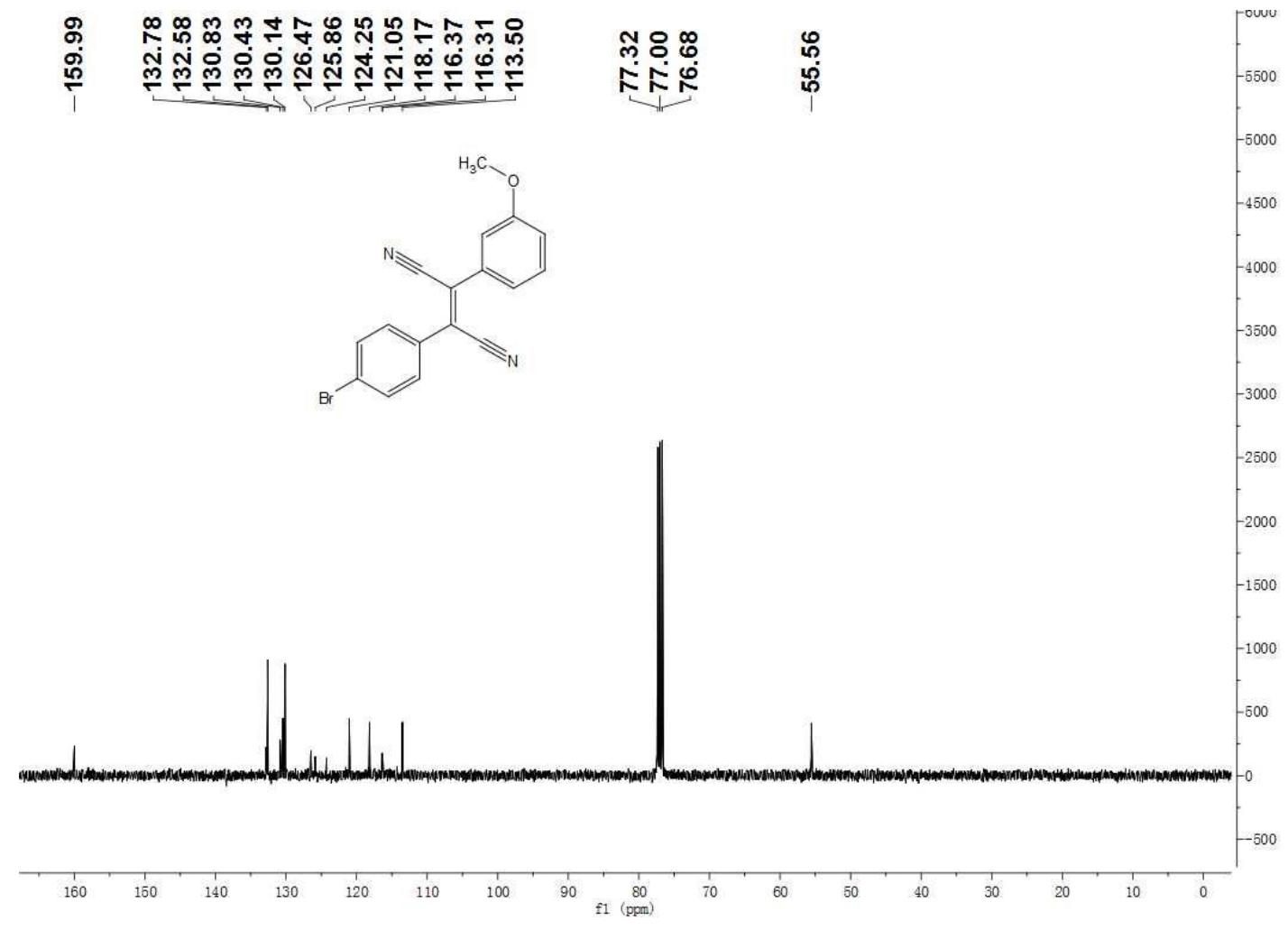

Figure S74. ${ }^{13} \mathrm{C}\left\{{ }^{1} \mathrm{H}\right\}$ NMR spectrum of $4 \mathrm{p}\left(100 \mathrm{MHz}, \mathrm{CDCl}_{3}\right)$ 


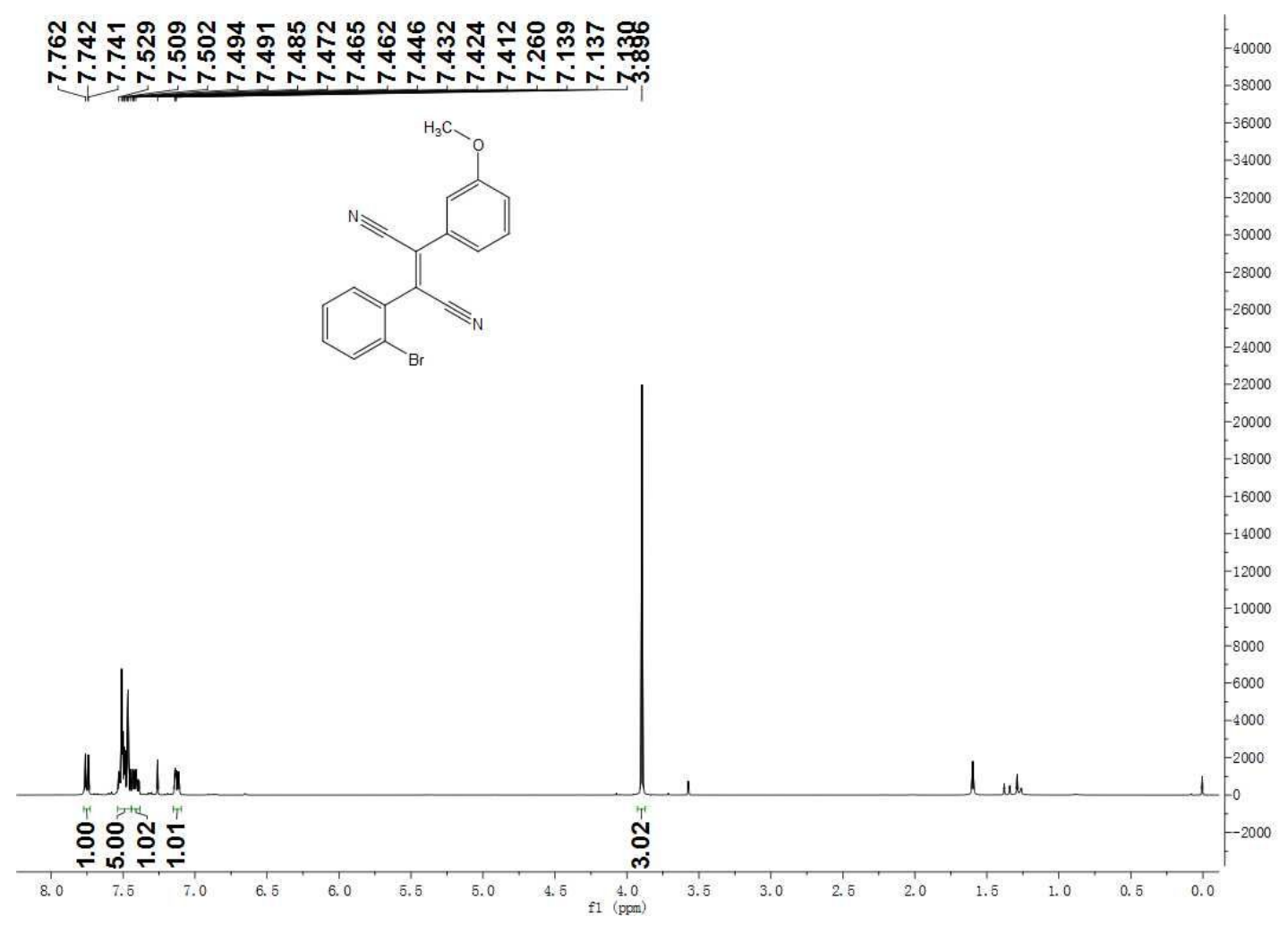

Figure S75. ${ }^{1} \mathrm{H}$ NMR spectrum of $4 \mathrm{q}\left(400 \mathrm{MHz}, \mathrm{CDCl}_{3}\right)$

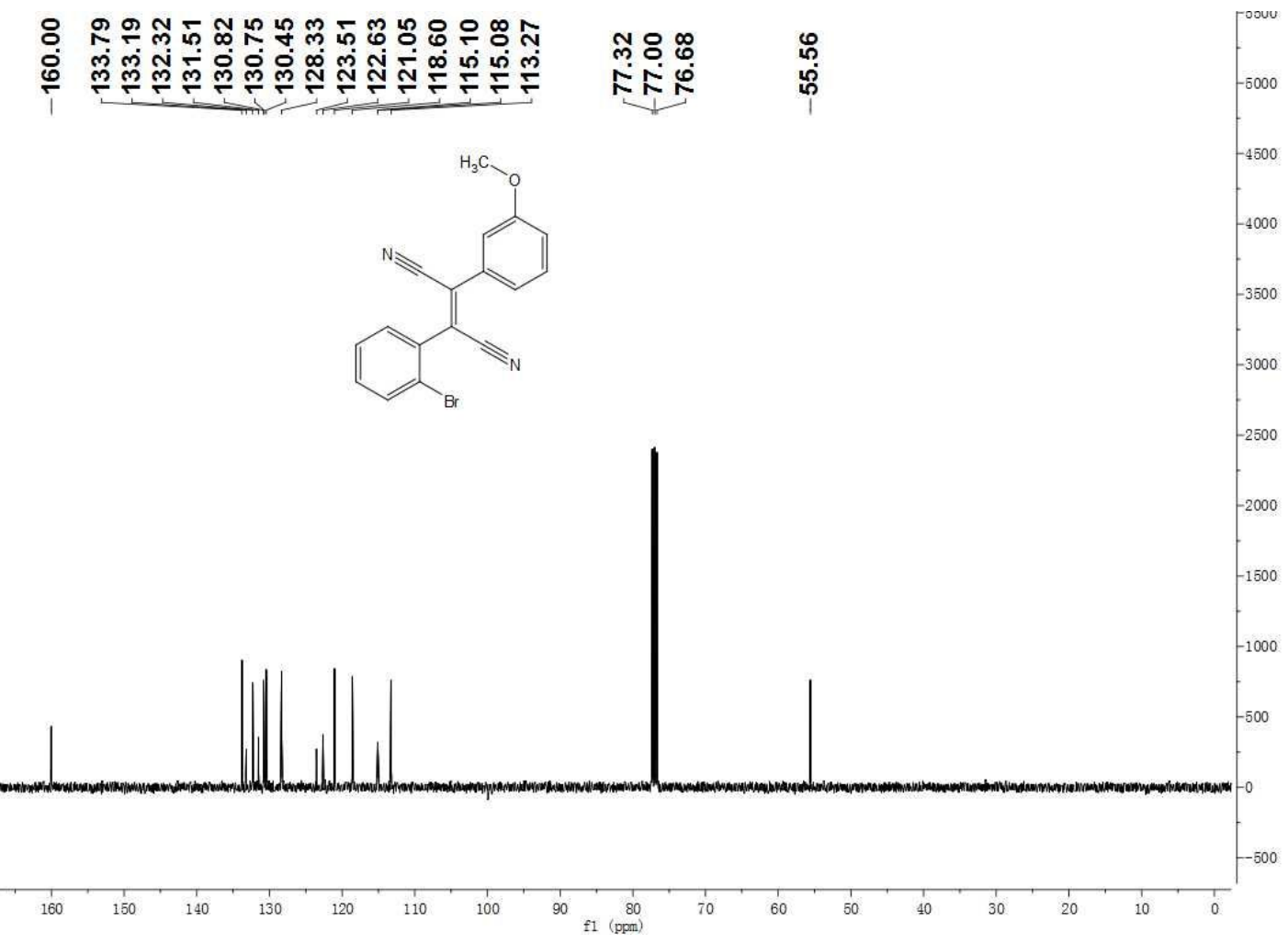

Figure S76. ${ }^{13} \mathrm{C}\left\{{ }^{1} \mathrm{H}\right\}$ NMR spectrum of $4 \mathrm{q}\left(100 \mathrm{MHz}, \mathrm{CDCl}_{3}\right)$ 


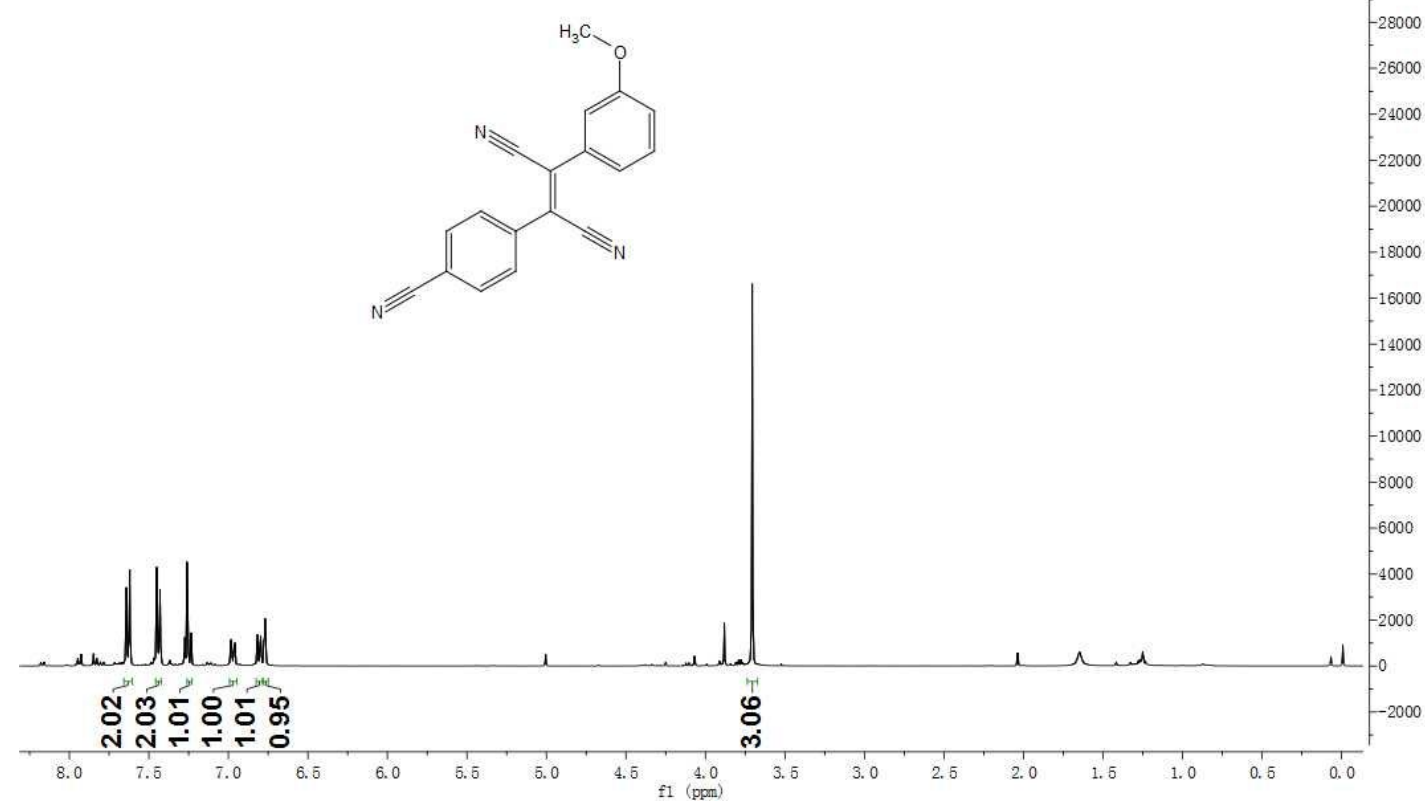

Figure S77. ${ }^{1} \mathrm{H}$ NMR spectrum of $4 \mathrm{r}\left(400 \mathrm{MHz}, \mathrm{CDCl}_{3}\right)$

โิ

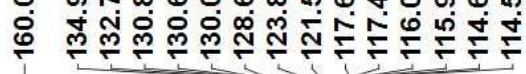

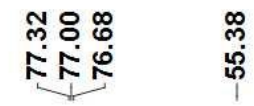

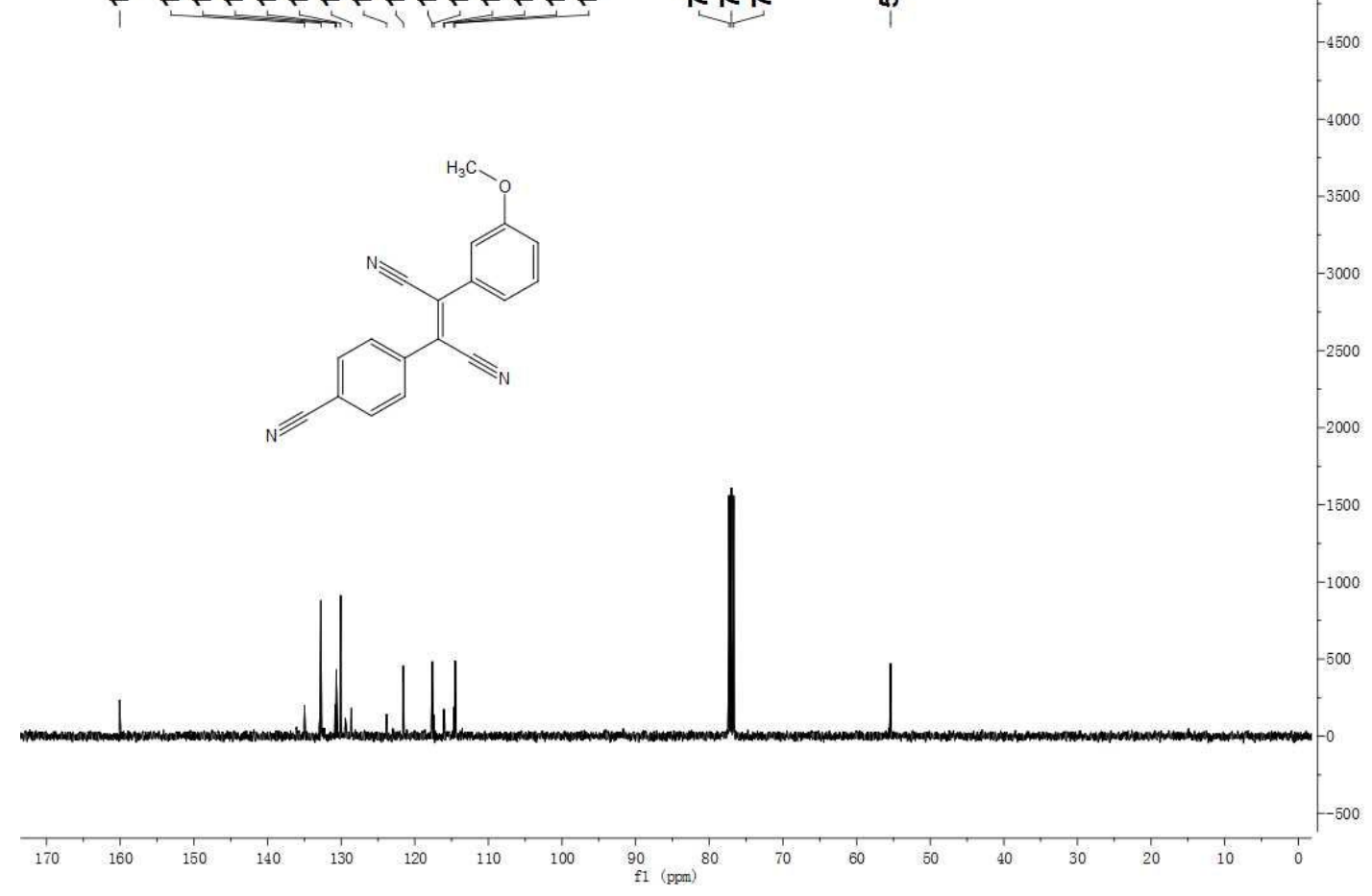

Figure S78. ${ }^{13} \mathrm{C}\left\{{ }^{1} \mathrm{H}\right\}$ NMR spectrum of 4 r (100 $\left.\mathrm{MHz}, \mathrm{CDCl}_{3}\right)$ 


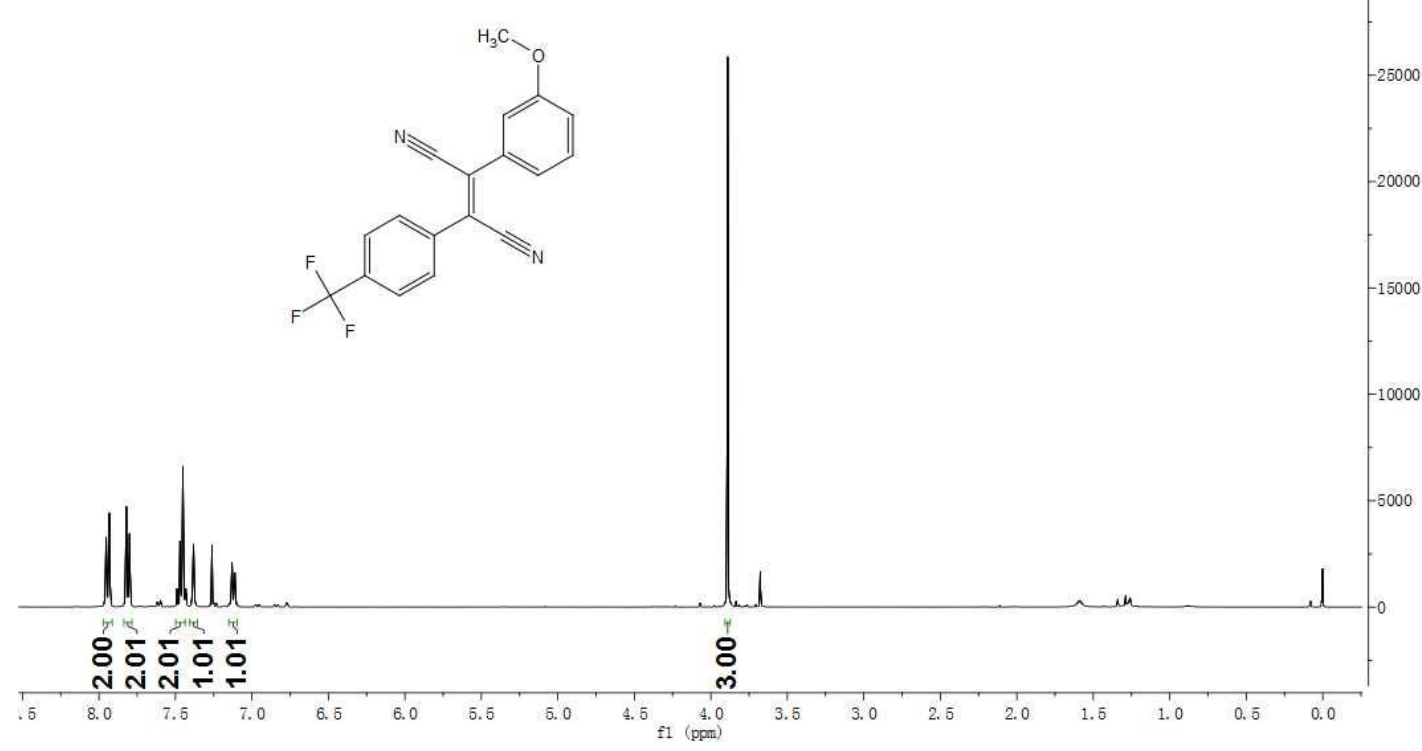

Figure S79. ${ }^{1} \mathrm{H}$ NMR spectrum of $4 \mathrm{~s}\left(400 \mathrm{MHz}, \mathrm{CDCl}_{3}\right)$

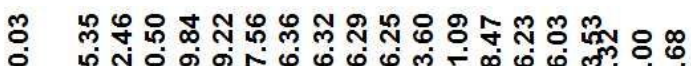

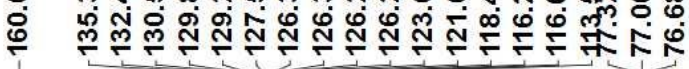

呫

(1)

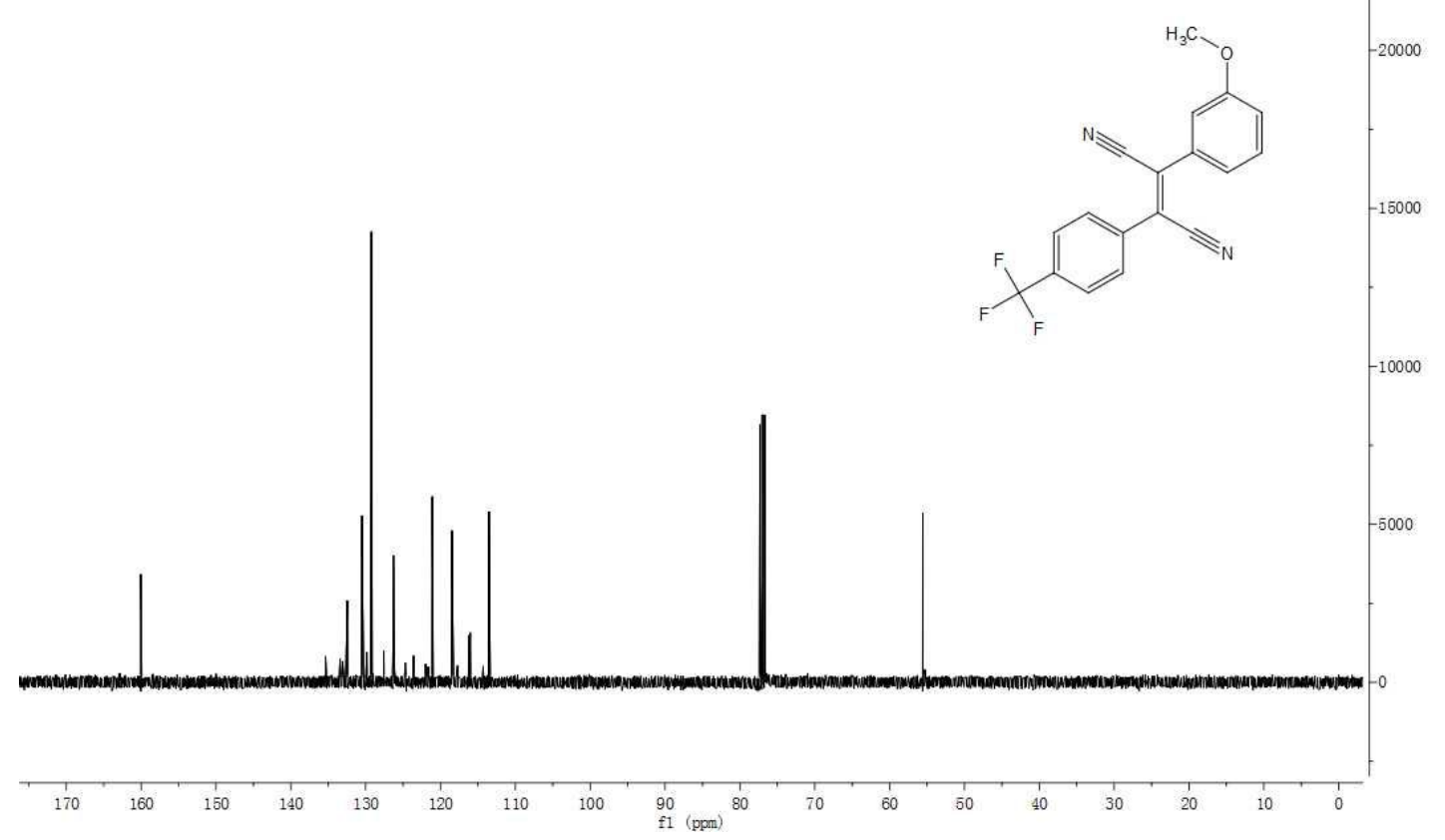

官

Figure S80. ${ }^{13} \mathrm{C}\left\{{ }^{1} \mathrm{H}\right\}$ NMR spectrum of $4 \mathrm{~s}\left(100 \mathrm{MHz}, \mathrm{CDCl}_{3}\right)$ 


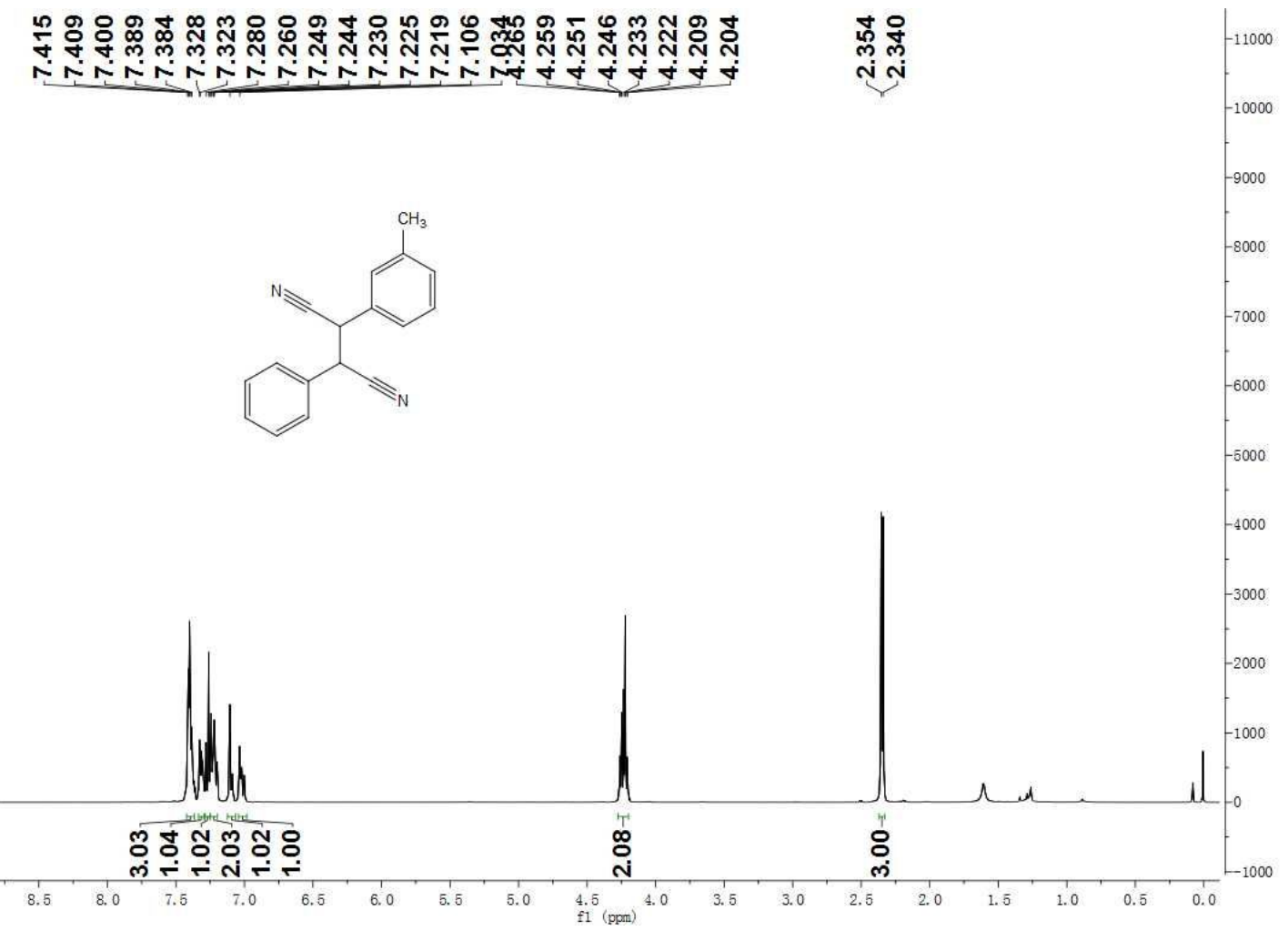

Figure S81. ${ }^{1} \mathrm{H}$ NMR spectrum of $5 \mathrm{a}\left(400 \mathrm{MHz}, \mathrm{CDCl}_{3}\right)$

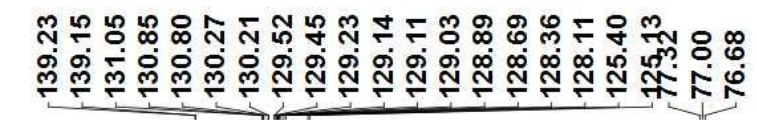

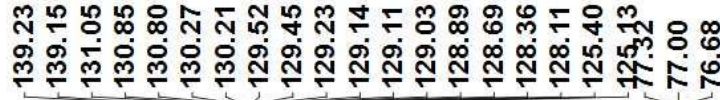
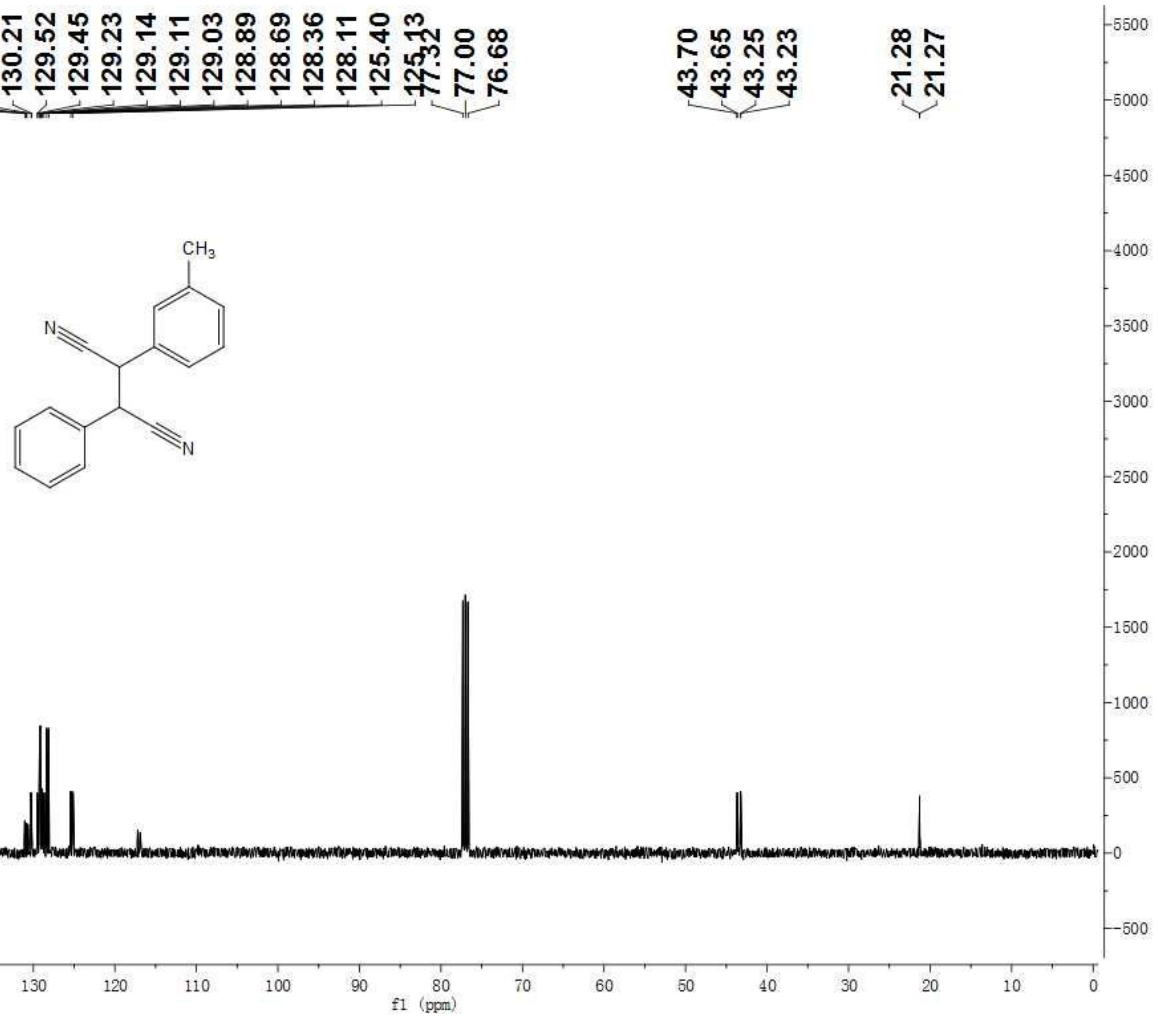

Figure S82. ${ }^{13} \mathrm{C}\left\{{ }^{1} \mathrm{H}\right\}$ NMR spectrum of $5 \mathrm{a}\left(100 \mathrm{MHz}, \mathrm{CDCl}_{3}\right)$ 


\section{X-ray crystallographic data}

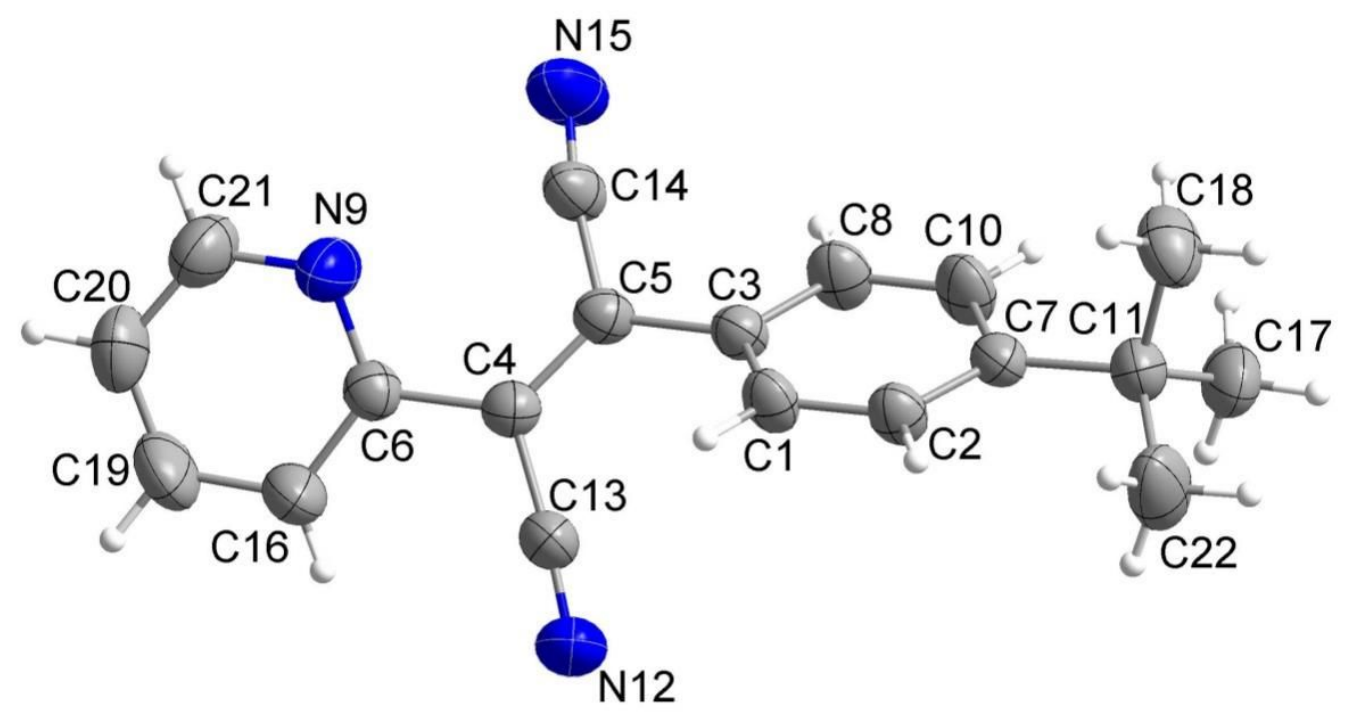

Figure S83. The Diamond diagram of 3e (thermal ellipsoids are shown at 50\% probability)

Sample Preparation: A crystalline solid was obtained via slow evaporation of 
compound 3e in EA: hexane $=1: 5$ at room temperature.

Crystal data and structure refinement for compound 3e (CCDC: 2094613)

Table S1 Crystal data and structure refinement for $3 \mathrm{e}$.

Identification code

Empirical formula

Formula weight

Temperature/K

Crystal system

Space group

$\mathrm{a} / \AA$

$\mathrm{b} / \AA$

$\mathrm{c} / \AA ̊$

$\alpha /{ }^{\circ}$

$\beta /{ }^{\circ}$

$\gamma /{ }^{\circ}$

Volume $/ \AA^{3}$

Z

$\rho_{\text {calc }} \mathrm{g} / \mathrm{cm}^{3}$

$\mu / \mathrm{mm}^{-1}$

$\mathrm{F}(000)$

Crystal size $/ \mathrm{mm}^{3}$

Radiation

$2 \Theta$ range for data collection $/{ }^{\circ}$

Index ranges

Reflections collected

Independent reflections

Data/restraints/parameters

Goodness-of-fit on $\mathrm{F}^{2}$

Final $\mathrm{R}$ indexes $[\mathrm{I}>=2 \sigma(\mathrm{I})]$

Final $\mathrm{R}$ indexes [all data]

Largest diff. peak/hole / e $\AA^{-3}$
$3 \mathrm{e}$

$\mathrm{C}_{38} \mathrm{H}_{35} \mathrm{~N}_{6}$

575.72

295.0

triclinic

P-1

$8.8905(4)$

$8.9236(4)$

$11.2632(5)$

$77.8142(15)$

$89.2730(17)$

$65.8849(16)$

794.49(6)

1

1.203

0.073

305.0

$0.2 \times 0.2 \times 0.2$

$\operatorname{MoK} \alpha(\lambda=0.71076)$

5.616 to 54.988

$-11 \leq \mathrm{h} \leq 11,-11 \leq \mathrm{k} \leq 11,-14 \leq 1 \leq 14$

11206

$3528\left[\mathrm{R}_{\text {int }}=0.0261, \mathrm{R}_{\text {sigma }}=0.0301\right]$

$3528 / 0 / 206$

1.071

$\mathrm{R}_{1}=0.0568, \mathrm{wR}_{2}=0.1284$

$\mathrm{R}_{1}=0.0744, \mathrm{wR}_{2}=0.1372$

$0.23 /-0.16$ 